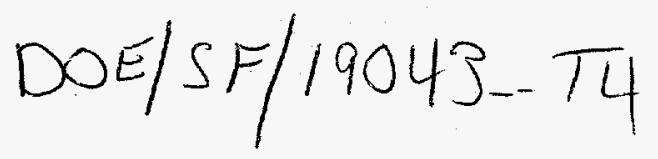

\title{
Nuclear Electric Propulsion for Future NASA Space Science Missions
}

Chen-wan L. Yen, Study-Leader

July 20, 1993

\author{
$J P L$ \\ Jet Propulsion Laboratory \\ California Institute of Technology 91109
}

JPL Publication D-10689

\section{DISCLAIMER}

This report was prepared as an account of work sponsored by an agency of the United States Government. Neither the United States Government nor any agency thereof, nor any of their employees, makes any warranty, express or implied, or assumes any legal liability or responsibility for the accuracy, completeness, or usefulness of any information, apparatus, product, or process disclosed, or represents that its use would not infringe privately owned rights. Reference herein to any specific commercial product, process, or service by trade name, trademark, manufacturer, or otherwise does not necessarily constitute or imply its endorsement, recommendation, or favoring by the United States Government or any agency thereof. The views and opinions of authors expressed herein do not necessarily state or reflect those of the United States Government or any agency thereof. 


\section{DISCLAIMER}

Portions of this document may be illegible in electronic image products. Images are produced from the best available original document. 
The research described in this publication was carried out by the Jet Propulsion Laboratory, California Institute of Technology, under a contract with the National Aeronautics and Space Administration and the Department of Energy. 
FINAL REPORT

TABLE OF CONTENTS

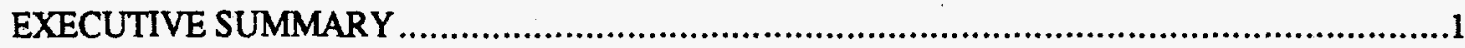

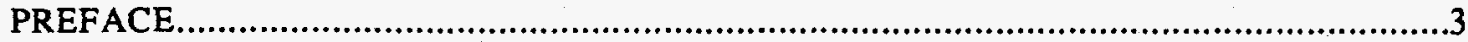

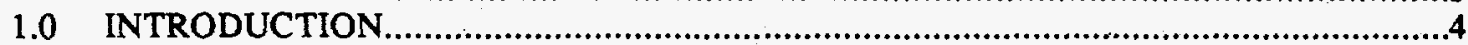

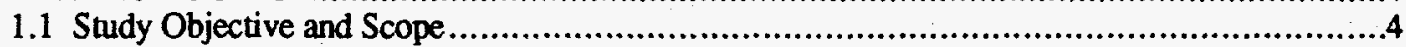

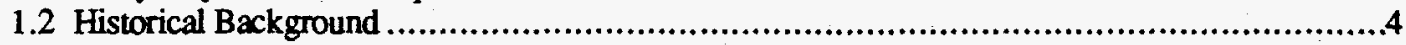

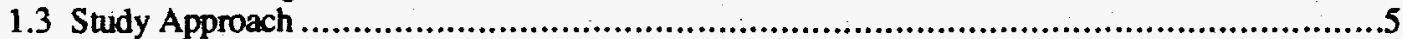

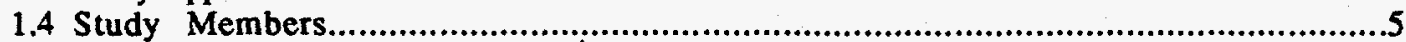

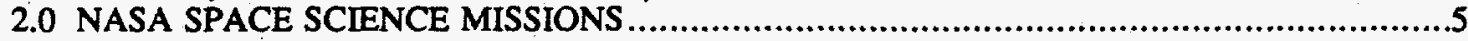

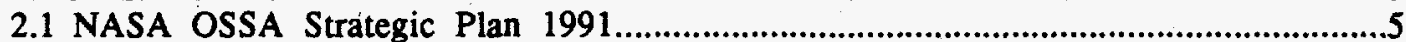

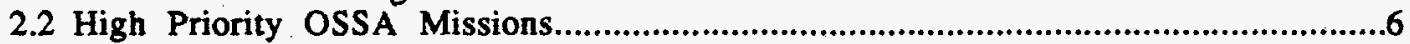

2.3 Limitations of Ballistic Mission Performance ...........................................................6

2.4 NEP Mission Concepts and Advantages.....................................................................

2.4.1 Propulsive Capability and Mass Performance.........................................................

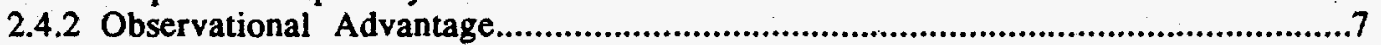

2.4.3 Flight Time Advantage and Frequent Mission Opportunities..........................................

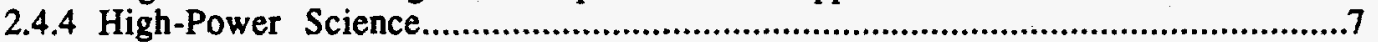

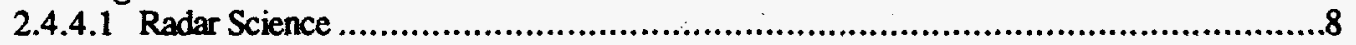

2.4.4.2 Non-radar High-Power Science ...................................................................8

2.4.5 Neutron Activation Science.........................................................................9

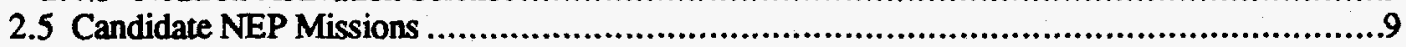

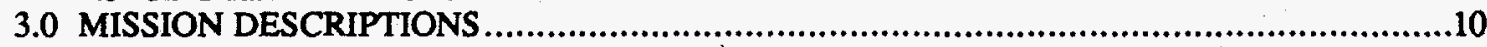

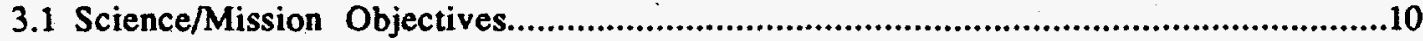

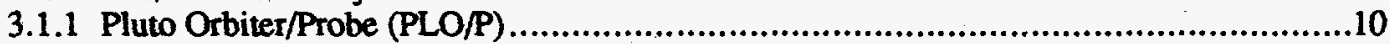

3.1.2 Neptune Orbiter/Probe (NEO/P) ......................................................................10

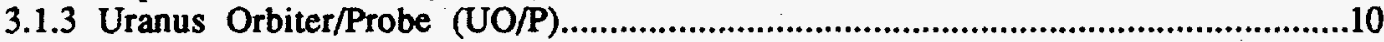

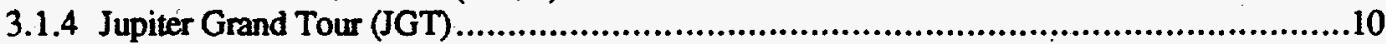

3.1.5 Multiple Mainbelt Asteroid Rendezvous (MMBAR) ..................................................11

3.1.6 Comet Nucleus Sample Return (CNSR) ...............................................................11

3.1.7 1-AU Solar Polar Orbiter (1-AU SPO) ….........................................................11

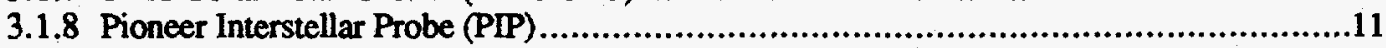

3.2 Planetary Mission Science Payloads...................................................................................12

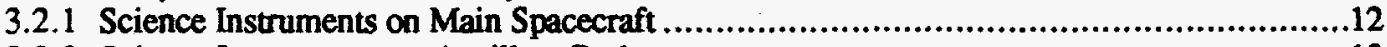

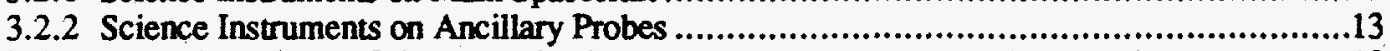

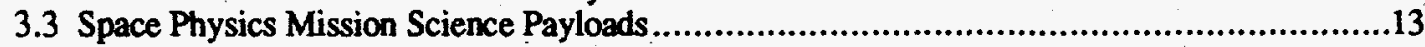

3.4 Planetary Mission Spacecraft................................................................................14

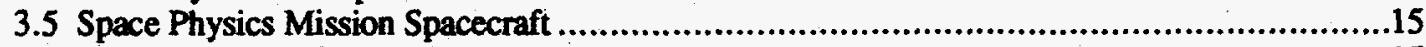

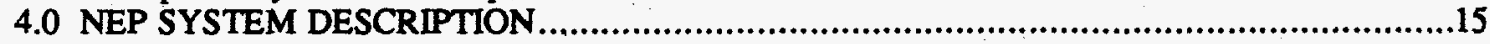

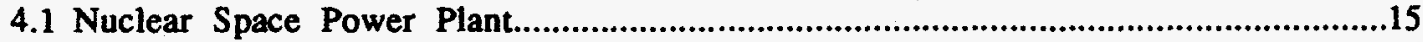

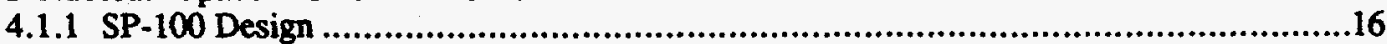

4.1 .2 Power Plant Mass vs. Power and Life ..............................................................17

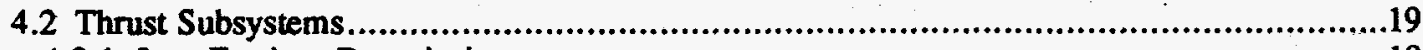

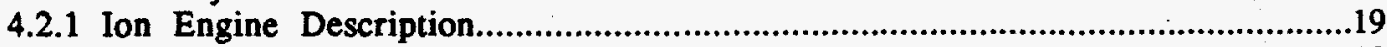

4.2.2 Developmental Status ..................................................................................19

4.2.3 Thruster Performance Characteristics ....................................................................20

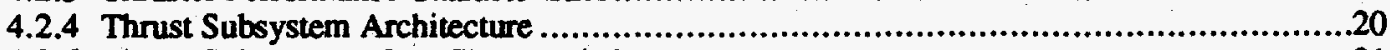

4.2.5 Thrust Subsystem Mass Characteristics ..............................................................21

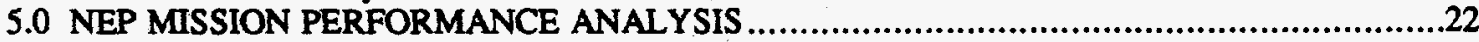

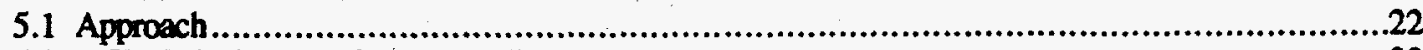

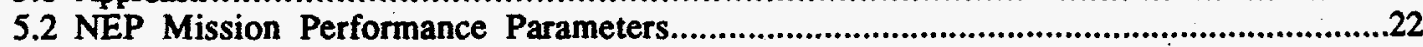

5.2.1 Launch Vehicles............................................................................................22

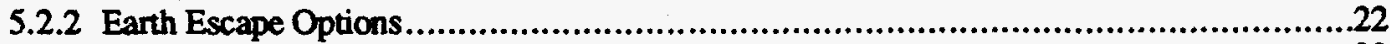

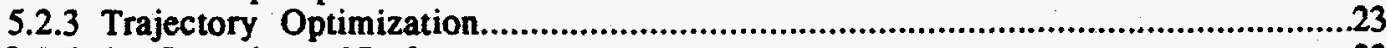

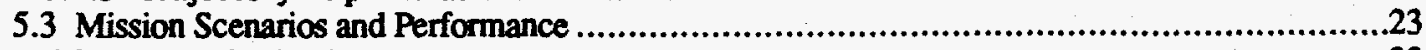

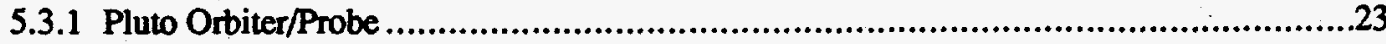




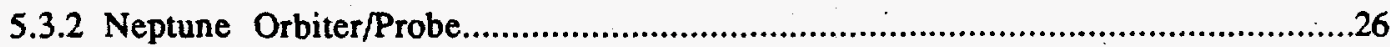

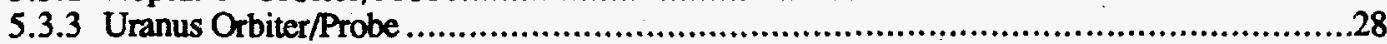

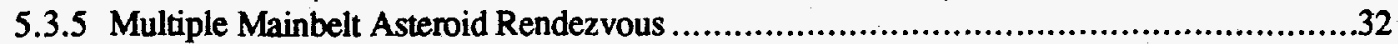

5.3.6 Comet Nucleus Sample Return ......................................................................34

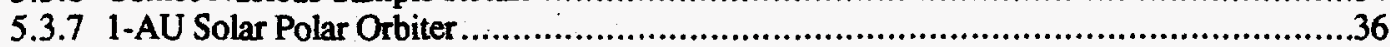

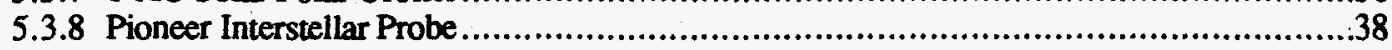

5.3.9 Performance Data (Tables) .................................................................................

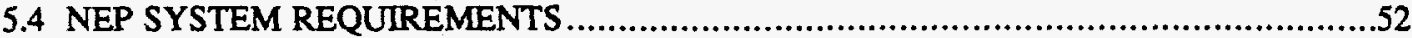

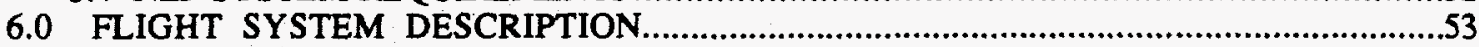

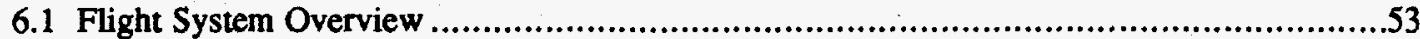

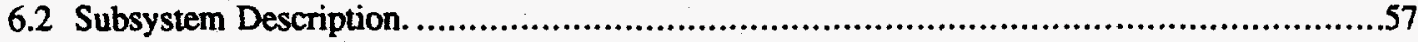

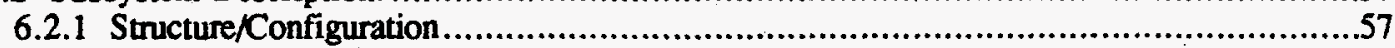

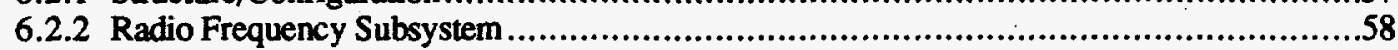

6.2.3 Power/Pyrotechnics ....................................................................................58

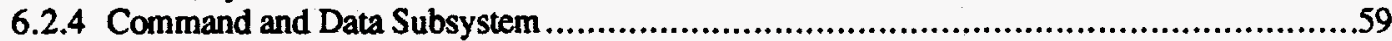

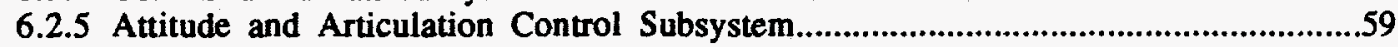

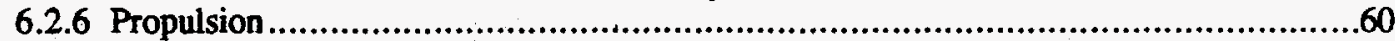

6.2.7 Thermal Control Subsystem.................................................................................6

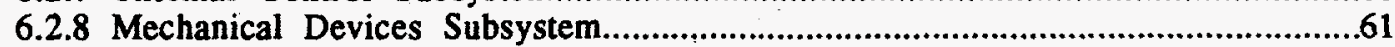

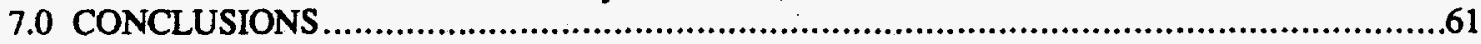

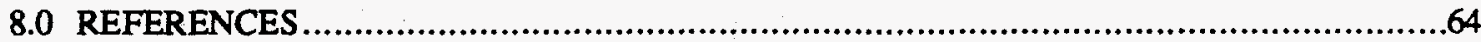




\section{EXECUTIVE SUMMARY}

\section{STUDY PERIOD March 1991 - July 1992}

FUNDING $\$ 200 \mathrm{~K}$ sponsored by NASA Office of Aeronautics And Space Technology, NASA Office of Space Science And Applications, and $\mathrm{DoE}$

\section{OBJECTIVES}

- Evaluation of applicability and benefits of Nuclear Electric Propulsion (NEP) technology circa year 2000 , on high priority NASA science missions.

- Establishment of key NEP system parameters and requirements.

- Provide comprehensive NEP vs. chemical propulsion mission/science comparative data for the planning of future US space science and technology programs.

\section{MISSIONS INVESTIGATED}

Pluto + Charon Orbiter, Neptune Orbiter with Probe, Uranus Orbiter with Probe, Jupiter Grand Tour, Multiple Mainbelt Asteroid Rendezvous, Comet Nucleus Sample Return, Jupiter Polar Orbiters, 1-AU Solar Polar Orbiter, and Pioneer Interstellar Probe.

\section{SPACECRAFT}

CRAF/Cassini Mariner Mark II derivatives for planetary missions. MMS spacecraft for 1-AU Solar polar Orbiter and a JPL design for Pioneer Interstellar Probe.

\section{REPRESENTATIVE NEP TECHNOLOGY CIRCA YEAR 2000}

- Power source: SP-100 technology ( $50-400 \mathrm{~kW}$, thermoelectric conversion, up to 10 year fullpower life)

- Thrusters: 30-cm Ring-Cusp thrusters with specific impulse range of 5000 to 10,000 seconds, assumed $10,000 \mathrm{hr}$ life

\section{RESULTS}

\section{Launch Vehicle Requirement}

- A Heavy Launch Vehicle (HLV), as characterized by the canceled Shuttle-C/Centaur or its potential replacement the National Launch System (NLS), is needed to fully realize the mission benefits that NEP technology can offer.

\section{General NEP Mission/Science Advantages}

- Comprehensive multiple-body science per mission; orbiter class exploration of a total planetary system including planet, satellites, rings and their environments.

- Payload mass and flight time advantages.

- Availability of substantial amounts of power for science and spacecraft subsystems.

\section{NEP System Requirements}

- $50 \mathrm{~kW}$ NEP with Titan IV(SRMU)/Centaur for Multiple Mainbelt Asteroid Rendezvous. 
- $100 \mathrm{~kW}$ NEP with HLV for all other missions investigated.

- A 10 year full-power and 15 year life for the SP-100.

- NEP system specific mass in the range of 50 to $55 \mathrm{~kg} / \mathrm{kW}$.

- An exception, a $>400 \mathrm{~kW}$ NEP with a $>25$ year life and a HLV are required for an Pioneer Interstellar Probe mission. Acceleration level attainable is insufficient (due to large specific mass), indicating the need for an advanced NEP for this mission.

\section{Key Differences between NEP and Chemical Mission Options}

- Outer Planets: Key difference is the science content. NEP enables a grand tour of a complete planetary system in rendezvous or slow flyby mode vs. fast flybys of satellites with inferior observational vantages offered by a loose elliptic orbit about the planet with a chemical option. NEP advantage is approximately 10-12 years vs. 15-20 years in flight time. Pluto is an exception in that the mission is not feasible without a NEP unless a long flight time, exceeding 35 years is acceptable.

- Multiple Mainbelt Asteroid Rendezvous: NEP offers more than six prime targets of interest in ten years, vs. 1 to 2 rendezvous with targets of opportunity in eight years, for chemical option.

- Comet Nucleus Sample Return: NEP offers frequent opportunities to sample high priority targets vs. marginal performance and accessibility dictated by rare gravity assist opportunities.

- 1-AU Solar Polar Orbiter: NEP enables the mission with a flight time of 7.5 years. Mission is not feasible with conventional chemical option.

- Interstellar Probe: Circa 2000 NEP can reach 200 AU in 22 years given a 1 MW power plant vs. 25 years using a solar grazing ballistic option. The need for an advanced NEP with much lower specific mass and long life is indicated. 


\section{PREFACE}

The focus of recent NEP (Nuclear Electric Propulsion) technology development programs has been on the needs of the Space Exploration Initiative (SED. In 1991 a shift in the program's direction emerged to include early applications of the technology to less demanding robotic planetary missions. The NEP technology and space science communities are in agreement that early applications of small NEP systems can be beneficial to science missions. The missions, relying on relatively mature technologies such as SP.

100 SRPS (Space Reactor Power System) and ion engines, can serve as the proving ground for the multimega watt advanced systems needed for the SEI.

With the sponsorship of NASA and DoE, a study was initiated to examine the needs of NASA core science programs and to evaluate the potential of NEP in performing important missions. Difficult missions with high science payoffs were selected as the logical candidates for NEP application. Evaluations were made to determine, 1$)$ the applicability of near term ( year 2000) NEP technologies, 2$)$ the mission benefits of NEP compared to conventional propulsion systems and 3) the key NEP system requirements. This study has been organized to provide an information link between the technology and science communities.

The premise of NEP applications to science missions, in this study, is that the missions will be of flagship class. This is in harmony with the inherent capability of NEP and costs.

The changes in NASA's science mission emphasis since 1992 represented by "faster, cheaper and better' theme is in stark contrast to the role that NEP may have in the space science. A set back to NEP mission prospects occurred as a result of the September 1992 Goldin/Watkins directive. The directive indicated the possible cancellation of SP-100 development program unless an attractive uniquely NEP enabled science mission can be nominated based on the technology of 1994. As the results of this study indicate, the unique and important role NEP can have for the planetary science program is in the exploration of the outer planets. A long-life, low mass NEP system is needed for this, and this is still a few years away from full readiness.

Although the current political and fiscal environment portends an uncertain future for NEP, this study provides a good faith analysis of what NEP can mean to NASA's planetary science program. As such, the results presented here should be a useful reference for the next step in the "NEP for Planetary Science" program, if and when NASA and DoE should decide to continue sponsorship or to revive the program. 


\subsection{INTRODUCTION}

\subsection{Study Objective and Scope}

A study has been made to assess the needs, potential benefits and the applicability of early (circa year 2000) Nuclear Electric Propulsion (NEP) technology in conducting NASA science missions. The study goals are:

1) to obtain the performance characteristics of near term NEP technologies

2) to measure the performance potential of NEP for important OSSA missions

3) to compare NEP performance with that of conventional chemical propulsion

4) to identify key NEP system requirements

5) to clarify and depict the degree of importance NEP might have in advancing NASA space science goals

6) to disseminate the results in a format useful to both NEP users and technology developers.

This is a mission performance study and precludes investigations of multitudes of new mission operation and systems design issues attendant in a NEP flight.

\subsection{Historical Background}

For more than two decades, many papers ${ }^{1-7}$ have been written promoting the superior performance possibilities of electric propulsion for planetary and space sciences, whether it be solar (SEP) or nuclear (NEP) powered. During the early stages (1960-1970) significant strides were made in the development of various trajectory optimization techniques ${ }^{8-13}$ (software), and by the early 1970's VARITOP (Sauer's code $^{12}$ ) and CHEBYTOP ${ }^{13}$ were used to design planetary missions ${ }^{3-7}$ yielding reliable results. In contrast, hardware (technology) development and proof of concept, in particular thruster life and reliability, have not attained similar levels of progress. The only program, SERT II ${ }^{14}$, aimed for the space test of thrusters in 1970 failed after 3800 hours of operation. The prospect of focused EP development generated by interests in missions to comet Halley ${ }^{15}$ in 1976-77 and SEPS (Solar Electric Propulsion Stage) to a comet Tempel-2 rendezvous with Halley flyby ${ }^{16}$ in 1979-80, was dashed when NASA discarded the pursuit of these missions. Development in the 1980's were limited to isolated technology development uncoupled from NASA science mission applications. During this period, the emphasis appeared to have been on defense applications (Strategic Defense Initiative) and on ambitious manned missions which brought about the rebirth of space nuclear reactor power in the form of the SP-100 project.

The implementation of the core science programs of the NASA Office of Space Science and Applications (OSSA), which could have benefited from the availability of SEP or NEP, proceeded with the use of conventional chemical rockets. Some difficult missions were made possible with clever applications of gravity assists, but mostly at the expense of longer flight times. The examples of important science missions made possible in the 1980's by gravity assists are Galileo (Jupiter Orbiter with Probe), CRAF (Comet Rendezvous/Asteroid Flybys) and Cassini (Saturn Orbiter with Titan Probe), Mainbelt Asteroid Rendezvous, Mercury Orbiter and outer planet missions with very long flight times. However, there is a limit to what gravity assists can accomplish and many of the advanced missions that are contemplated by NASA for the next decade are in need of non-conventional delivery systems, such as NEP, or other innovative flight system design approaches such as micro-spacecraft.

In recent years, the Space Exploration Initiative (SEI) spurred renewed interests in high technologies needed for conducting ambitious robotic and manned explorations of the Moon and Mars. Many space nuclear power and propulsion concepts have been proposed and studied in the U.S. to meet these mission requirements ${ }^{17}$. Of these, technologies of the SP-100 Space Reactor Power System (SRPS) ${ }^{18}$ and the Ion Engine ${ }^{19}$ are viewed as sufficienuly mature to be viable candidates, requiring relatively modest incremental development, for application to future science missions. This is also a logical step, as the precursor, in aiding the development of the multi-mega watt NEP needed for the SEI. 
Unlike SEP flights of earlier attempts, use of nuclear power is expected to introduce many new technical challenges and political ones as well. Significant changes to the mission scenarios and flight system design are expected. One previous SP-100 Mission/System design study ${ }^{20}$ conducted at JPL presents a fairly comprehensive set of design issues related to space science missions and provides preliminary approaches in addressing the problems.

\subsection{Study Approach}

It is presumed that pertinent issues of NEP in OSSA strategic planning processes are to understand: 1) how necessary is NEP and what differences can NEP make in performing a mission, 2) what are the required technologies, 3) what is the technology readiness level and 4) the implied risks and the costs. This study addresses the first two issues with a comprehensive set of important missions and technology assumptions, representing the forecasted technology readiness level of the year 2000 . Objectives, mission scenario and performance of each candidate NEP mission are analyzed in sufficient depth to provide a fair comparison to the chemical option with regard to the overall quality of the mission.

In the above process the NEP technology required to meet the demands of these missions are identified. They may serve as a reference technology development goal for the robotic science missions.

\subsection{Study Members}

Contributors to the study are listed in Table 1-1.

Table 1-1. NEP Mission Study Team Members

(All at JPL unless otherwise noted)

\section{Mission Design}

Chen-Wan L. Yen

Rodica lonasescu

Carl G. Sauer

Kurt Hack (LeRC)

John Reel (LeRC)

Henry M. Harris

\section{Spacecraft_Design}

Robert N. Mostert

-. Flight Systems Engineering

\section{NEP System}

Henrik G. Groniroos

John R. Brophy

Charles E. Garner
-. Study Lead and Mission Design

-- Science/Mission Scenario Design

-- Mission Performance Analysis

-- Mission Performance Analysis

-- Mission Performance Analysis

-. Documentation

\subsection{NASA SPACE SCIENCE MISSIONS}

\subsection{NASA OSSA Strategic Plan 1991}

In 1991 the NASA Office of Space Science and Applications (OSSA) published a Strategic Plan ${ }^{21}$ for the US space science and applications program. The Plan, which exemplifies the yearly OSSA mission 
planning process, established a process or set of actions that seek to provide a clear, coherent strategy that meets both NASA's and OSSA's goals. The Plan provides for programmatic themes, decision rules and a set of priorities for programs and missions within each theme.

The Plan describes goals for the dawn of the 21 st century that include the study of comets and asteroids as well as every accessible major body of the Solar System. A major conclusion of the Plan is that a strong technological base will be needed to realize these goals

Although NEP technologies have been under development in the US for some time, and potential merits of NEP applied to space science missions are generally recognized among the science community and OSSA, so far no cohesive and focused technology developer-user relationship has been established to make NEP an integral part of the core science strategy described in the Strategic Plan. Thus the planning of difficult high priority missions has been based on the use of conventional chemical rockets albeit with various drawbacks (less than satisfactory science return, limited payload, long flight time etc.) imposed by such a system.

This study examines the potential role of NEP in the context of OSSA long range goals.

\subsection{High Priority OSSA Missions}

The mechanisms by which OSSA establishes mission priorities are the Strategy Implementation Study Workshops held annually under their sponsorship. References 22 and 23 provide example profiles of the 1990-91 strategic planning processes by sub-divisions of OSSA; Solar System Exploration Sub-division (Code-SL) and Space Physics Sub-division (Code-SS). The fundamental OSSA science goals are firmly established through these and other similar workshops, and have remained unwavering for many years. They are used as the guiding principle in generating mission concepts and establishing mission priorities. The fundamental goals are summarized below:

- Observe the universe with high sensitivity and resolution across the entire electromagnetic spectrum.

- Complete the detailed scientific characterization of virtually all of the solar system including the terrestrial planets, primitive bodies and at least the nearer parts of the outer solar system. Develop the scientific foundation to support the planning of human exploration beyond Earth.

- Quantitatively describe the physical behavior of the Sun, the heliosphere, the interstellar medium and the Galaxy.

- Establish a set of observing platforms for observing the Earth on a global scale.

- Determine, develop, and exploit the unique capabilities to be provided by Space Station Freedom.

Various missions have been proposed under OSSA guidance over time which have had the expressed objective of realizing the stated goals. As one might expect, a set of missions has emerged that reflect these goals and have remained important fixtures within the OSSA program. The design concept of a particular mission (possessing the same name or derivative names) may differ in scale, details of implementation and in degree of public and official appeal due to various circumstances. The concept of a high priority mission set in OSSA is well understood. It is the view of the study participants that application of NEP must be in the context of a well established OSSA program and that it is important to insure that high quality science be returned that is commensurate with the inherent investment implied by NEP.

\subsection{Limitations of Ballistic Mission Performance}

The most pertinent parameter of a chemical rocket, one that bounds its performance capability, is its specific impulse (ISP). The specific impulse is proportional to the velocity (C) of the reaction mass in the exhaust of a rocket; i.e. $C=I S{ }^{*} 9.9 \mathrm{~m} / \mathrm{s}$ and the typical value of $C$ is $3-4 \mathrm{~km} / \mathrm{s}$ for an ISP of $300-400$ seconds. In simple terms, a space mission is implemented by imparting a sequence of velocity change 
maneuvers $(\Delta V s)$; Earth escape maneuver, deep space maneuvers and orbit capture maneuvers at target planet and so forth. A mission's performance is dictated by an exponential law, the rocket equation $\mathbf{M}_{\mathrm{f}}=$ $\mathrm{M}_{0}{ }^{*} \exp (-\Delta \mathrm{V} / \mathrm{C})$; where $\mathrm{M}_{\mathrm{f}}$ is the mass at the end of a mission, requiring total maneuvers of magnitude $\Delta \mathrm{V}$ and $\mathrm{M}_{0}$ is the mass at the beginning of a mission (e.g. the mass at Earth-departure parking orbit). The degree of difficulty is represented by the $\Delta V$ incurred in a mission and the exponential performance equation shows that the mission return, $\left(\mathrm{M}_{\mathrm{f}}\right)$ becomes poor using such a device if $\Delta \mathrm{V}$ becomes larger than two or three times the magnitude of $\mathrm{C}$. Given the conventional rocket, possible missions are confined to ones with $\Delta V \leq 7-10 \mathrm{~km} / \mathrm{s}$. Ambitious missions designed in recent years such as Galileo (Jupiter Orbiter), CRAF (Comet Rendezvous with Asteroid Flybys) and Cassini (Satum Orbiter with Titan Probe) are missions with $\Delta V$ less than $7 \mathrm{~km} / \mathrm{s}$ and represent near limit capabilities of the current chemical propulsion systems for that class of science missions. The $\Delta \mathrm{V}$ requirements of these missions would have been two to three $\mathrm{km} / \mathrm{s}$ greater were it not for the various gravity assists incorporated in the trajectory design. Note, however, the penalty incurred by the gravity assist---flight times are increased by more than three years. The orbiter class missions to the outer planets Uranus, Neptune and Pluto and others, if made feasible by brute force, posses undesirable attributes (extremely long flight times, small payloads, limited science etc.) and are in need of benefits to be gained by advanced propulsion systems such as NEP.

\subsection{NEP Mission Concepts and Advantages}

\subsubsection{Propulsive Capability}

The most attractive attribute of NEP is its ability to maneuver with great fuel efficiency. The high ISP (3000 - 10,000 seconds) of ion engines will enable difficult missions with high $\Delta V$ requirements 10 to 30 times larger than those possible with chemical rockets. Thus attaining tight circular orbits, changing a flyby encounter with a planetary body in a chemical option to an orbiter or rendezvous encounter, or achieving multiple rendezvous are the most distinctive mission enhancements expected of NEP. Pluto rendezvous is the best example of a mission enabled by NEP because of its propulsive advantages.

\subsubsection{Observational Advantage}

Once at the planet, an NEP spacecraft can begin a powered spiral down to lower orbits, with considerable freedom to select orbital characteristics according to investigative needs; such as comprehensive coverage of the environment, rendezvous or low speed encounters with satellites and rings etc. In more subtle ways the quality of science data returned during the planetary phase of the exploration is greatly improved due to NEP's ability to place a spacecraft in advantageous viewing positions (e.g. station-keeping with interesting features) returning high space and time resolutions.

\subsubsection{Flight Time Advantage and Frequent Mission Opportunities}

The mass characteristics of the NEP systems represented results in unatractive low acceleration levels $\left(\sim 0.1 \mathrm{~mm} / \mathrm{s}^{* *} 2\right)$ and the common expectation of greatly accelerated mission accomplishments cannot be realized in most cases. A flight time advantage of NEP over the ballistic option, for instance in outer-planet missions where flight time is a critical issue, is possible because the time consuming gravity assists are unnecessary (minimum of 3 years expended in gravity assists). In contrast to chemical options, the preclusion of gravity assists with NEP also means that launch opportunities occur with normal frequency (e. g. every synodic period for planets with low eccentricity).

\subsubsection{High-Power Science}

Limited by the capacity of conventional power sources (solar, RTG, batteries), almost all deep space science today, either in use or in the design stage, carries no more than a few hundred watts. With a nuclear power source this limitation is removed and offers the opportunities to include science investigations not possible to consider thus far. Although no specific high power experiments were incorporated in the mission scenarios to be presented later, examples of potentially attractive high power science are described 
in the following. Creation or inclusion of experiments taking advantages of the large nuclear power capacity perhaps will emerge when the availability and viability of NEP is demonstrated.

\subsubsection{Radar Science}

Past surveys 24,25 of the science community on the use of available high power indicated that the most favored mode of utilization is in the radar sciences. High-powered radar is a NEP-enabled science, especially in the outer reaches of the solar system where other conventional power systems become impractical. The science possibilities of high-powered radar can be divided into the categories of search and imaging which can also be dual operating modes of a hypothetical NEP-powered radar. The search mode would look for small undiscovered bodies from the vantage point of the mission locations such as the asteroid belt or outer-planet space. Upon orbital capture or flyby of bodies, the radar would switch to a SAR mode for detailed synthetic aperture imaging.

Various configurations may be considered for a radar mounted NEP spacecraft. Although it is possible to use the HGA (high gain antenna) for synthetic radar imaging (e.g. Cassini, Magellen), having a flat multi-frequency array has advantages in effective use of gain and data rate. Also, a flat array beam can be electronically steered which would be impossible for a circular HGA. A flat array would require a rigid structure of about a tenth of a wavelength tolerance. An array made of carbon-epoxy would have the strength and stiffness required for a SAR.

An important feature of a NEP SAR would be a dual mode transmitter/receiver that could transmit at very long wavelengths. Long wavelength SAR allows penetration into the icy surface of satellites and comets to a depth of as much as several kilometers. Hidden internal features would be revealed as changes in the dielectric constant. Some theories suggest that some satellites, such as Europa may be heated internally by tidal interaction and may contain interior liquid water. Ice/water interfaces could be mapped if they exist.

SAR has the capability to map very small (centimeter) changes in surface height such as would be produced by ice cracks and depositions by geysers on the surface. Any dynamic activity could be studied in detail by mapping the surface over time.

SAR could also be used at asteroids to map centimeter scale surface structure topography and measure the dielectric constant of the surface. If the asteroid was solid rock there will be no penetrations but some asteroids might be covered with a loose material that could be penetrated with radar to reveal underlying features.

\subsubsection{Non-radar High-Power Science}

High-powered laser technology is now mature, benefiting from decades of DOD research. Laser bombardment of asteroids and comets from a NEP spacecraft could be used to kick up dust and gas for capture and in-siw analysis. If the body has an atmosphere, the laser can be used for spectrographic analysis. High power will increase the depth of penetration.

Many science instruments can benefit in subtle ways from the availability of high power. Fields and particles instruments can have broader spectrums and increased number of frequencies. Radio science can benefit from deeper penetration of planetary atmospheres. Multi-link Doppler radio systems can have better resolution of gravity wave signatures and could theoretically detect gravity waves from weaker sources. Other relativity experiments could benefit by allowing deeper penetrations of RF signals into the solar gravity well.

Active cooling is another possibility although active cooling has not been used on planetary spacecraft in the past since there has been little demand for cryogenic temperatures and reliable long-life active coolers were not available. High technology experiments of the future may well require active cooling as superconducting electronics come on line or if high-powered communications or radar electronics demand it. 
were not available. High technology experiments of the future may well require active cooling as superconducting electronics come on line or if high-powered communications or radar electronics demand it.

Science instruments in the NEP radiation environment may also require active cooling. An infrared spectrometer is susceptible to damage done by neutron scattering from particles from the reactor and would require active temperature regulation to anneal solid-state electronics to minimize radiation damage. $\mathrm{CCDs}$ for imaging and optical navigation would also require active cooling to $-120 \mathrm{C}$ because of silicon displacement and ionization damage to the detector.

\subsubsection{Neutron Activation Science}

As part of this study, the possibility of utilizing the neutrons emitted by the reactor for scientific purposes was considered by $\mathrm{H}$. Harris (JPL), prompted by the fact that conventional gamma ray experiments are not viable in the nuclear environment and that neutron beams are used on the ground for the analysis of the elemental composition of matter.

Conventional gamma ray spectroscopy relies on cosmic rays or solar flare induced nuclear cascades to investigate the chemical composition of planetary bodies. Slow neutrons in the cascade particles are mostly responsible for generating gamma rays characteristic of the composition of matter.

Harris suggested that it is possible to open a small hole in the reflector surrounding the NEP reactor and produce a beam of neutrons. The beam could be collimated by a crystal material placed over the hole. This beam could be directed toward a target and the resulting gamma ray emissions recorded by a gamma ray spectrometer that is separated from the spacecraft, either in orbit or on the surface of the target body. Mounting the gamma ray spectrometer directly on the spacecraft would probably not be possible because it would be overwhelmed by gamma rays from the reactor.

An analysis of the idea was performed by Dr. Albert Metzger (JPL) for this study. He found that the reactor must be no more than 2 to 3 kilometers away from the target body to achieve the same results expected using cosmic ray neutrons. However, if the neutron source is 1 kilometer in distance (or landed) there is a significant science advantage. At this distance very accurate elemental composition of the body can be determined that is not possible using any other method. In addition, the number of elements that can be detected increases substantially.

\subsection{Candidate NEP Missions}

A set of candidate missions for NEP applications was chosen from the roster of missions included in NASA OSSA strategic planning workshops conducted in the past few years. Most of these missions have been categorized repeatedly as highly desirable but difficult to implement. Driven by strong scientific interests, the design of some of these missions using conventional rockets have proceeded, but the results have been mostly unsatisfactory to the mission planners in that they entail long flight times, insufficient payload deliveries and offered less than satisfactory science returns. Table 2-1 lists missions of primary interest to the Solar System Exploration Division (first six) and to the Space Physics Division (last two) of OSSA.

Table 2-1 NEP Mission Candidates

1. Neptune Orbiter/Probe (NEO/P)

2. Pluto Orbiter/Probe (PLO/P)

3. Uranus Orbiter/Probe (UO/P)

4. Jupiter Grand Tour (JGT)

5. Multiple Mainbelt Asteroid Rendezvous (MMBAR)

6. Comet Nucleus Sample Return (CNSR)

7. 1-AU Solar Polar Orbiter (1-AU SPO)

8. Pioneer Interstellar Probe (PIP) 


\subsection{MISSION DESCRIPTIONS}

\subsection{Science/Mission Objectives}

Science rationale and mission goals for missions listed in Table 2-1 have been established over the years of mission planning and science working team workshops. The following descriptions provide the representative thinking and the favored theme with respect to each of the missions.

\subsubsection{Pluto Orbiter/Probe (PLO/P)}

The planet Pluto and its satellite Charon form a dual planetary system due to their comparable masses. The atmosphere of Pluto is tenuous and at its maximum expansion near perihelion. As Pluto recedes from perihelion, its atmosphere will freeze out. It is also believed that Charon shares Pluto's atmosphere by pulling it away from Pluto as in a binary system, yet lacking the gravitational capability to maintain it around its own body.

The science goals for this mission are : 1) to study the geology, internal structure, surface composition and atmosphere-surface interactions on Pluto; 2) to map the surface composition and geology of Charon, and to determine whether surface processes or geologic features may have resulted from gravitational interactions between Pluto and Charon; 3) to determine the dynamics and composition of Pluto's atmosphere before atmospheric collapse (between 2020 and 2025) and 4) to conduct in-situ science with probes/soft landers on Pluto and Charon.

A desirable mission to Pluto would orbit Pluto and Charon and deploy a lander on one or both, if possible.

\subsubsection{Neptune Orbiter/Probe (NEO/P)}

This mission ranks high among the NASA planetary exploration programs. It is a logical sequence to follow the orbiter class exploration of Jupiter (Galileo) and Saturn (Cassini).

The fundamental science objectives of NEO/P are: 1) to characterize the structure, composition and dynamics of Neptune's atmosphere; 2) to study the geology, surface composition, atmosphere and atmosphere-surface interactions of Triton ; 3) to study the geology and composition of other satellites and 4) to determine the nature, composition and dynamics of the ring system and the interaction of the ring material with Neptune's magnetosphere.

A desired mission consists of the delivery of an atmospheric probe followed by an orbiter class exploration of Triton. Attaining favorable orbit for probing of other satellites, magnetosphere, ring system and for long term observation of the atmosphere are other desirable mission scenarios.

\subsubsection{Uranus Orbiter/Probe (UO/P)}

The science objectives of the Uranus mission are similar to the Neptune mission and include studies of the Uranus atmosphere, its satellites and ring systems.

\subsubsection{Jupiter Grand Tour (JGT)}

The "Jupiter Grand Tour" is a NEP based mission concept in which the objective of orbiter observations of four Galilean satellites is to be realized in a single launch. A satellite orbiter riding on a NEP is to successively orbit about Callisto, Ganymede, and Europa and potentially Io (if the radiation problem can be managed). Additional rendezvous or slow encounters of this spacecraft with other satellites are also expected.

A more ambitious concept 22 includes the addition of Jovian space physics exploration (Jupiter Polar Orbiter mission-JPO) involving two fields and particles spacecraft. This option is not set as the primary 
mission goal in this study, but in cases where an excess performance margin exist, additions of landers or JPO spacecraft are considered.

The satellite science objectives include morphology, geology and physical state characterization of surfaces; surface mineralogy and elemental composition distribution; thermophysical properties; gravity and magnetic fields; and satellite interaction with the Jovian magnetosphere. Landers will facilitate in-situ satellite measurements, as for example, local imaging and seismic activity monitoring.

The objectives of the JPO's are to study Jupiter aeronomy, auroral physics, atmosphere processes, ring structure, gravity fields, Io aeronomy, Io torus interaction and coupling, energetic particle processes and radio astronomy.

\subsubsection{Multiple Mainbelt Asteroid Rendezvous (MMBAR)}

The science goals for this mission are to determine the asteroid size, shape, rotation, albedo, mass, density, surface morphology, surface composition, magnetic fields, and interaction with the solar wind. Since the asteroid population is diverse (in sizes, physical and compositional characteristics and their distance from the sun), a scientifically meaningful asteroid mission would require sampling of a sufficiently large number of diverse classes of asteroids. This is the overriding requirement imposed by the asteroid science community for the design of a MMBAR mission.

\subsubsection{Comet Nucleus Sample Return (CNSR)}

The primary objective of a CNSR mission, similar to ESA/NASA ROSETTA mission ${ }^{34}$, is to return to Earth pristine samples of comet surface material, core material (maintain temperature $<160^{\circ} \mathrm{K}$ ) and volatiles for in-depth laboratory study. The cold, volatile-rich matter of a comet is thought to contain the most primitive samples of condensed protoplanetary material in the solar system and are valuable in providing clues about the primordial condition and processes leading to the formation of the solar system. The desired target is an active relatively "new" comet. High resolution imaging and in-situ examination of the sample site are other important science goals.

\subsubsection{1-AU Solar Polar Orbiter (1-AU SPO)}

The 1-AU solar polar orbiter is an element of a network of solar monitoring spacecraft that will simultaneously perform remote sensing and in-situ measurements from multiple vantage points that will provide fundamental new data for understanding the solar interior, the solar atmosphere, the solar wind and the solar magnetic cycle.

This mission addresses the need to understand the variable nature of the Sun, the short-term variations associated with the evolution of magnetic activity cycle, and the magnitude and significance of long-period solar variability and its influence in the heliosphere. The mission would be devoted primarily to solarphysics remote sensing but could also carry out systematic exploration of high-latitude solar wind, magnetic field, and energetic particles, thus building on the initial reconnaissance carried out by Ulysses.

The polar view of the sun will allow accurate measurements of the polar magnetic field, differential rotation profile, and meridional flow free from the extreme projection effect of viewing from the Earth.

\subsubsection{Pioneer Interstellar Probe (PIP)}

The Pioneer Interstellar Probe mission described herein differs from the previous TAU (Thousand AU) mission concept that focused on astronomical objectives. The pioneer Interstellar Probe will have its prime focus on fields and particles. The scientific objectives will be to 1) explore the nature of the interstellar medium and its implications for the origin and evolution of matter in the galaxy; 2) explore the structure of the heliosphere and its interaction with the interstellar medium; and 3) explore fundamental astrophysical processes occurring in the heliosphere and interstellar medium. 
The primary goal of the mission would be to pass through the solar wind termination shock, the heliopause and the bow shock and penetrate interstellar medium. In situ measurements of the magnetic field, the plasma, the galactic cosmic rays, the neutral gas and dust with versions of instruments tailored to probe the outer heliosphere and interstellar medium are desired.

Although the size of the heliosphere is uncertain, present estimates place the termination shock at $\sim 100$ to $150 \mathrm{AU}$ from the Sun. To attain the above objectives the spacecraft will be targeted towards the leading edge of the heliosphere at right ascension $70^{\circ}$, declination $7^{\circ}$ with a goal of reaching $200 \mathrm{AU}$ within 25 years or less.

It is possible that one or more of the Pioneer or Voyager spacecraft may locate the termination shock and retum valuable data within their lifetimes but the instrumentation's of these earlier probes cannot accommodate the measurement needs of outer heliosphere science. At present Pioneer 10 is $52 \mathrm{AU}$ from the sun heading toward the heliospheric tail. Its end of life is predicted in 1996 at $\sim 65$ AU. Pioneer 11 is 33 $\mathrm{AU}$ from the Sun and nearing the end of its life. Voyager 1 and 2 are 46 and $38 \mathrm{AU}$ away. Voyager 1 and 2 are heading towards the general direction of solar apex at $35^{\circ}$ north and $48^{\circ}$ south latitude respectively. The traveling speed of the Voyagers are roughly 3.5 AU/year. The Voyagers are expected to partially function until 2012 when they reach $\sim 130$ and 107 AU respectively.

\subsection{Planetary Mission Science Payloads}

\subsubsection{Science Instruments on Main Spacecraft}

Table 3-1 is a matrix of strawman science instruments on board the main spacecraft versus the missions. The total mass of the science payload ranges from 153 to $217 \mathrm{~kg}$, depending on the mission.

Table 3-1 Matrix of Main Spacecraft Science Payload vs. Mission

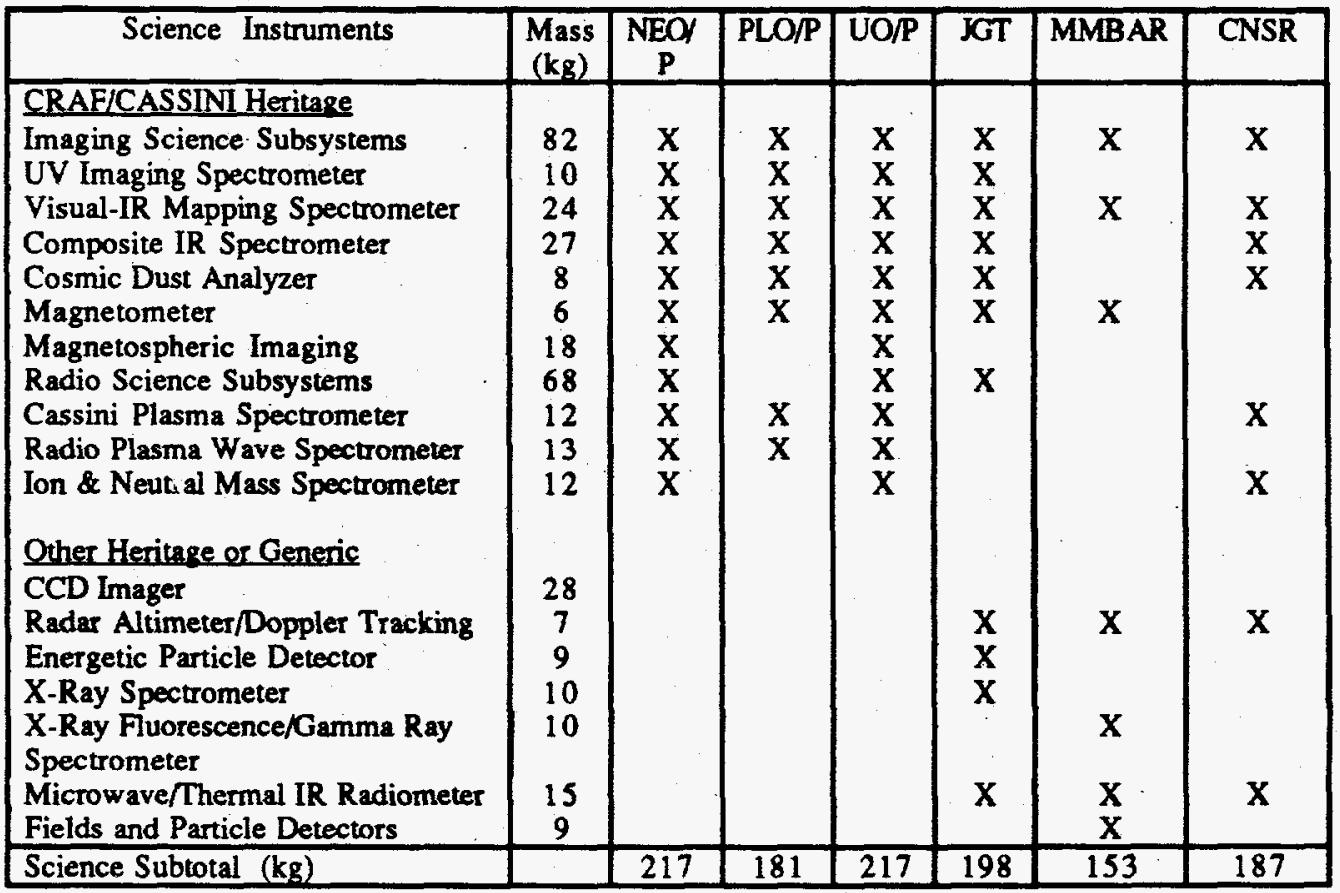




\subsubsection{Science Instruments on Ancillary Probes}

Example science instruments on various other probes; e.g. Neptune and Uranus atmosphere probes, small landers (= penetrators) for Triton and Jovian satellites, and large landers for Triton and Pluto and/or Charon are given in Tables 3-2 through 3-6. These are secondary payloads, that may be included on an "if possible" basis.

Table 3-2. Candidate Uranus/Neptune Probe Science Instruments

\begin{tabular}{|l|r|}
\hline Probe Instruments & $\begin{array}{r}\text { Mass } \\
(\mathrm{kg})\end{array}$ \\
\hline Atmosphere Structure Instrument (ASI) & 3.8 \\
Nephelometer (NEP) & 4.6 \\
Helium Abundance (HAD) & 1.4 \\
Net Flux Radiometer (NFR) & 3.0 \\
Neutral Mass Spectrometer (NMS) & 11.2 \\
Lightring and Radio Emission & 2.3 \\
(LRD)/Energetic Particles (EPI) & \\
Radio Science & 6.3 \\
Gas Chromatograph (GC) & 7.0 \\
\hline Science Subtotal & 37.3 \\
\hline
\end{tabular}

Table 3-3. Candidate Penetrator Instruments for Outer Planet Satellites

\begin{tabular}{|l|c|}
\hline \multicolumn{1}{|c|}{ Penetrator Instruments } & $\begin{array}{c}\text { Mass } \\
(\mathrm{kg})\end{array}$ \\
\hline Seismometer & 0.60 \\
Alpha Proton Backscatter/X-Ray & 0.40 \\
Fluorescence Spectrometer & \\
Temperature Sensors & 0.07 \\
Water Detector & 0.15 \\
Accelerometer & 0.03 \\
Surface Imaging & 0.25 \\
Magnetometer & 0.40 \\
\hline Science Subtotal & 1.90 \\
\hline
\end{tabular}

Table 3-4. Candidate Penetrator Instruments for Asteroids

\begin{tabular}{|l|c|}
\hline \multicolumn{1}{|c|}{ Penetrator Instruments } & $\begin{array}{c}\text { Mass } \\
(\mathrm{kg})\end{array}$ \\
\hline Gamma Ray Spectrometer & 8.70 \\
Temperature Probe Assembly & 0.50 \\
Accelerometer Sensor Group & 0.20 \\
Surface Imaging & 0.25 \\
Magnetometer & 0.40 \\
\hline Science Subtotal & 10.05 \\
\hline
\end{tabular}

Table 3-5. Candidate Lander Instruments for Pluto/Charon

\begin{tabular}{|l|c|}
\hline Lander-Probe Instruments & $\begin{array}{c}\text { Mass } \\
(\mathrm{kg})\end{array}$ \\
\hline Surface Sampler & 13.0 \\
Multi-Spectral Surface Imaging & 5.0 \\
Magnetometer & 0.4 \\
Alpha Proton Backscatter/X-Ray & 2.0 \\
Fluorescence Spectrometer & \\
Scanning Electron Microscope/Microprobe & 12.0 \\
X-Ray Diffractometer & 5.0 \\
Seismometer & 2.2 \\
Petrographic Microscope & 5.0 \\
Temperature Sensors & 0.1 \\
Atmosphere Structure Instrument & 3.8 \\
Nephelometer & 4.6 \\
Net Flux Radiometer & 3.0 \\
Ion and Neutral Mass Spectrometer & 12.4 \\
\hline Science Subtotal & 68.5 \\
\hline
\end{tabular}

Table 3-6. Candidate Lander Instruments for Triton and Galilean Satellites

\begin{tabular}{|l|c|}
\hline \multicolumn{1}{|c|}{ Lander Instruments } & $\begin{array}{c}\text { Mass } \\
(\mathrm{kg})\end{array}$ \\
\hline Surface Sampler & 13.0 \\
Multi-Spectral Surface Imaging & 5.0 \\
Magnetometer & 0.4 \\
Alpha Proton Backscatter/X-Ray & 2.0 \\
Fluorescence Spectrometer & \\
Scanning Electron Microscope/Microprobe & 12.0 \\
X-Ray Diffactometer & 5.0 \\
Seismometer & 2.2 \\
Petrographic Microscope & 5.0 \\
Temperature Sensors & 0.1 \\
\hline Science Subtotal & 44.7 \\
\hline
\end{tabular}

\subsection{Space Physics Mission Science Payloads}

Space physics is the study of the sun, heliosphere, solar wind, and cosmic rays, and their interactions with the upper atmospheres, ionspheres, and magnetospheres of the planets and comets, and with the interstellar medium, as one system. Table 3-7 gives an example space physics payload for the 1- AU Solar Polar and Pioneer Interstellar Probe missions. 
Table 3-7 Matrix of Space Physics Instruments vs. Mission

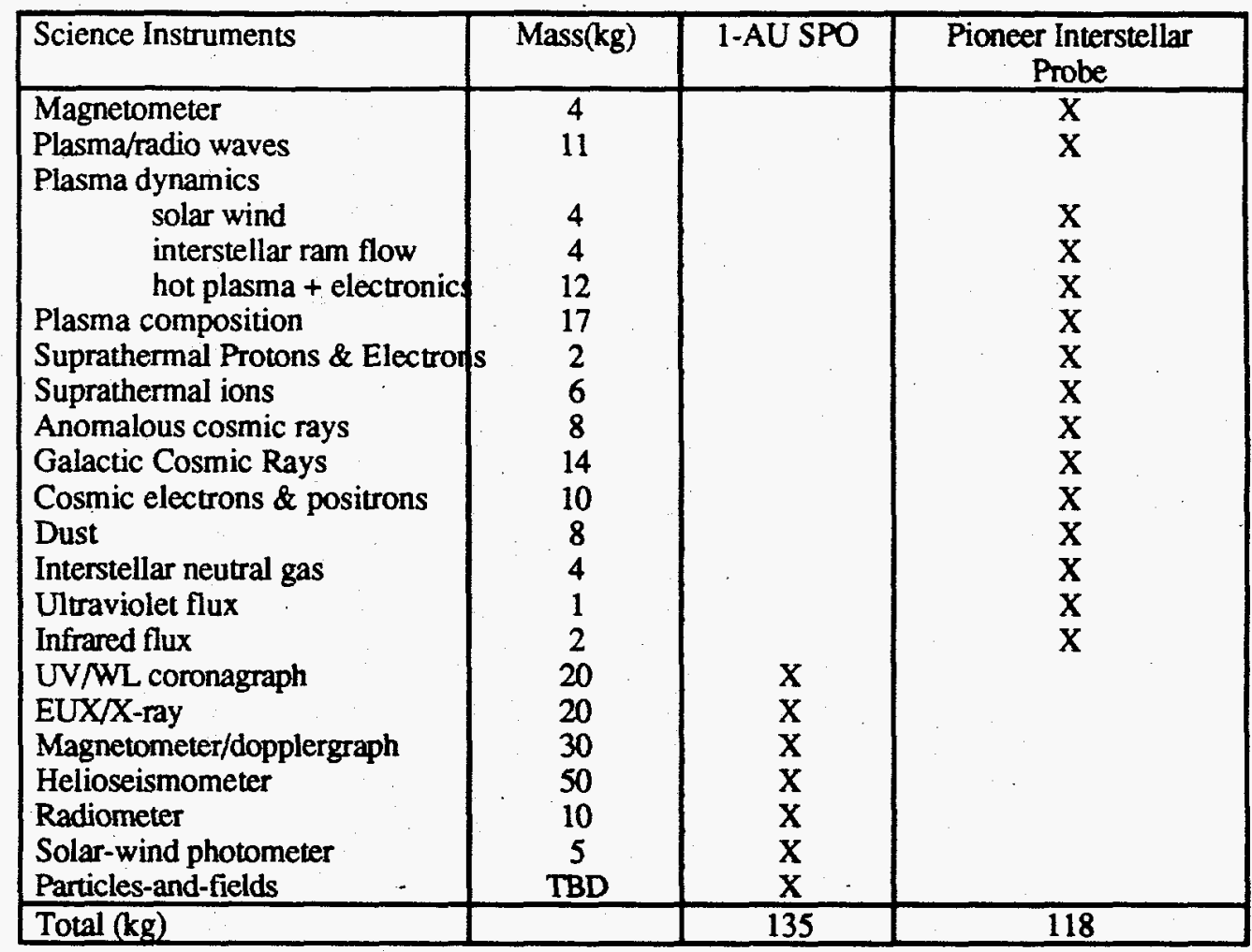

\subsection{Planetary Mission Spacecraft}

The main spacecraft concept for each of the planetary missions is derived, with appropriate modifications, from the Mariner Mark II (MMk II); the spacecraft originally designed for the now canceled CRAF and CASSINI (which also is scaled down in scope at this writing) missions. Adaptation to NEP means removal, from the original MMK II, of the on board chemical propulsion system, RTG, reaction wheels and associated electronics. A preliminary investigation of a comprehensive set of issues accompanying a NEP-spacecraft system has been addressed in 1986 by Jones et al. ${ }^{20}$. A number of configurational options and attitude control mechanisms were suggested in that study. Although each concept possessed certain problems, it was concluded that integration of a nuclear power plant, an electric propulsion system and a typical planetary spacecraft is possible using current or projected technical capabilities. Concerns for the spacecraft health and the science interference implied by the use of a nuclear reactor and the ion engine are some of the key questions awaiting further investigation.

The total mass of the MMK II derived mission spacecraft is basically $1500 \mathrm{~kg}$ for all missions. Small variations among the missions are due to the differences in supporting differing science payloads as shown in Table 3-8. Mass estimates include a $30 \%$ contingency.

\section{Ancillary Probes}

The masses of the suggested secondary payloads (atmospheric probe, small lander = penetrator, medium size lander etc.) are also given in Table 3-8. Propulsion requirements for the probes differ from mission to mission depending on the planet it is targeted for and how the probe is to be deployed. For landers, the deployment is assumed to be initiated from a $500 \mathrm{~km}$ circular orbit of the targeted body. Table 3-3 shows the masses of the probe both with and without the propulsion system. The $\Delta V$ required and the type of chemical propulsion assumed are also shown. For the bi-propellant type, a specific impulse of 300 seconds, a tankage factor of $16 \%$ and an inert mass of $60 \mathrm{~kg}$ is used to size the chemical propulsion system. For the solid propulsion type, an inert mass equal to $10 \%$ of the propellant is assumed. 
Table 3-8. Matrix of Potential Payloads vs. Mission

\begin{tabular}{|c|c|c|c|c|c|c|c|}
\hline Mission & & NEO/P & PLO & $00 / P^{\circ}$ & fGi & MIVIBAR & CNSR \\
\hline $\begin{array}{c}\text { Main } \\
\text { Spacecraft }\end{array}$ & $\begin{array}{l}\text { science }(\mathrm{kg}) \\
\text { mass wo/prop. }(\mathrm{kg}) \\
\text { mass w/prop. }(\mathrm{kg}) \\
\Delta v(\mathrm{~km} / \mathrm{s}) \\
\text { prop. type }\end{array}$ & $\begin{array}{l}217 \\
1410\end{array}$ & $\begin{array}{c}181 \\
1410\end{array}$ & $\begin{array}{c}217 \\
1410\end{array}$ & $\begin{array}{c}198 \\
1434\end{array}$ & $\begin{array}{r}153 \\
1361\end{array}$ & $\begin{array}{l}187 \\
1395\end{array}$ \\
\hline $\begin{array}{c}\text { Atmosphere } \\
\text { Probe }\end{array}$ & $\begin{array}{l}\text { science }(\mathrm{kg}) \\
\text { mass wo/prop. }(\mathrm{kg}) \\
\text { mass w/prop. }(\mathrm{kg}) \\
\Delta v(\mathrm{~km} / \mathrm{s}) \\
\text { prop. type }\end{array}$ & $\begin{array}{c}37 \\
376 \\
370 \\
0 . \\
\text { none }\end{array}$ & & $\begin{array}{c}37 \\
376 \\
1081 \\
2.3 \\
\text { bi-pro } \\
\end{array}$ & & & \\
\hline * Lander & $\begin{array}{l}\text { science }(\mathrm{kg}) \\
\text { mass wo/prop. }(\mathrm{kg}) \\
\text { mass w/prop. }(\mathrm{kg}) \\
\Delta \mathrm{v}(\mathrm{km} / \mathrm{s}) \\
\text { prop. type }\end{array}$ & $\begin{array}{c}45 \\
384 \\
862 \\
1.6 \\
\text { bi-pro }\end{array}$ & $\begin{array}{c}69 \\
415 \\
742 \\
1.1 \\
\text { bi-pro }\end{array}$ & & $\begin{array}{c}45 \\
384 \\
946-1197 \\
1.8-2.3 \\
\text { bi-pro }\end{array}$ & & $\begin{array}{c}26 \\
533\end{array}$ \\
\hline * Penetrator & $\begin{array}{l}\text { science }(\mathrm{kg}) \\
\text { mass wo/prop. }(\mathrm{kg}) \\
\text { mass w/prop. }(\mathrm{kg}) \\
\Delta \mathrm{v}(\mathrm{km} / \mathrm{s}) \\
\text { prop. type }\end{array}$ & $\begin{array}{c}2 \\
50 \\
97 \\
1.6 \\
\text { solid }\end{array}$ & $\begin{array}{c}2 \\
50 \\
79 \\
1.1 \\
\text { solid }\end{array}$ & $\begin{array}{c}2 \\
50 \\
64-125 \\
0.6-2.1 \\
\text { solid }\end{array}$ & & 11 & \\
\hline $\begin{array}{l}\text { Earth Retum } \\
\text { Vehicle }\end{array}$ & $\begin{array}{l}\text { science }(\mathrm{kg}) \\
\text { mass wo/prop. }(\mathrm{kg}) \\
\text { mass w/prop. }(\mathrm{kg}) \\
\Delta \mathrm{v}(\mathrm{km} / \mathrm{s}) \\
\text { prop. type }\end{array}$ & & & & & & $\begin{array}{c}0 \\
321 \\
396 \\
0.1\end{array}$ \\
\hline $\begin{array}{c}\text { F* Equivalent } \\
\text { Final Orbit } \\
\text { (Planet Radii) }\end{array}$ & & 8.0 & & 1.5 & 3.5 & $=1.0$ & $\begin{array}{l}\text { retum to } \\
\text { earth with } \\
V_{\infty}=0\end{array}$ \\
\hline $\begin{array}{l}\text { Basic Mission } \\
\text { Requirements }\end{array}$ & $\begin{array}{l}\text { mass in final orbit } \\
(\mathrm{kg}) \\
\text { Probe delivered en } \\
\text { route }(\mathrm{kg})\end{array}$ & $\begin{array}{l}1410 \\
376\end{array}$ & 1410 & $\begin{array}{l}1410 \\
1081\end{array}$ & 1434 & 1361 & 1791 \\
\hline
\end{tabular}

Foptional payload * See 5.2 .3 for explanation.

\subsection{Space Physics Mission Spacecraft}

The concept for a solar physics mission is derived from the Solar Maximum Mission (SMM) which is a solar observatory mounted on a Multimission Modular Spacecraft (MMS). The MMS is a standard NASA spacecraft recently used successfully on TOPEX/POSEIDON. The MMS houses the essential components of the three spacecraft subsystems: attitude control, power, and communications and data handling.

The SMM-MMS components, the SMM payload configuration, and the solar observatory weighs about $2500 \mathrm{~kg}$ and this number was used for study purposes for a 1-AU Solar Polar Mission using NEP.

The Interstellar Probe spacecraft is based on the design by T. Penn (JPL). Details of the design are described in an internal Jet Propulsion Laboratory Document ${ }^{39}$. This design uses readily available technology with substantial design heritage from Pioneer 10/11, Voyager, Galileo and MMII. The spacecraft (without large chemical propulsion) weighs $1000 \mathrm{~kg}$.

\subsection{NEP SYSTEM DESCRIPTION}

\subsection{Nuclear Space Power Plant}

The SP-100 program is the only near-term space reactor power system developmental program existing in the U.S. today. The SP-100 system transforms reactor thermal power to electricity by thermoelectric conversion. This system has been under development since 1986: The goal is to develop by the end of the 1990s a safe, long life and high reliability nuclear power plant adaptable for a broad range of space missions. 
The SP-100 program is sponsored by three different U.S. Government agencies, the Department of Energy, the Department of Defense, and the National Aeronautics and Space Administration. The program has successfully evolved into its current Power System Qualification phase. This phase will, through component and assembly testing and verified analytical methods, demonstrate the technology for building a space flight system scaleable over electrical power levels ranging from tens to hundreds of kilowatts.

The progress of the SP-100 system design and technology program has been reported at several technical conferences $26,27,18$ The following summarizes the major features of the baseline SP-100 generic flight system design and the modifications made to that design to arrive at a reduced mass system for interplanetary nuclear electric propulsion (NEP) science mission applications.

\subsubsection{SP-100 Design}

There are eight subsystems. The functional and configurational drawings of the SP-100 GFS (Generic Flight System) are shown in Figure 4-1.1 and 4-1.2 respectively. The GFS is designed to meet generic functional requirements enveloping a range of space missions. The fast-neutron spectrum reactor is made of niobium base alloy and contains uranium nitride fuel. A set of actuators and controls, automatically or through command position reflector control elements and safety rods to start up and maintain the system at constant power. A shadow shield of lithium-hydride and depleted uranium encased in stainless steel attenuates both neutrons and gamma radiation. Six liquid metal (lithium) heat transport piping loops made of niobium alloy transfer heat from the reactor to twelve thermoelectric conversion assemblies (SiGe/GaP) by self-actuated thermoelectromagnetic pumps. Twelve heat rejection loops made of titanium piping transport waste heat to radiator panels with titanium-potassium heat pipes armored with carbon-carbon material.

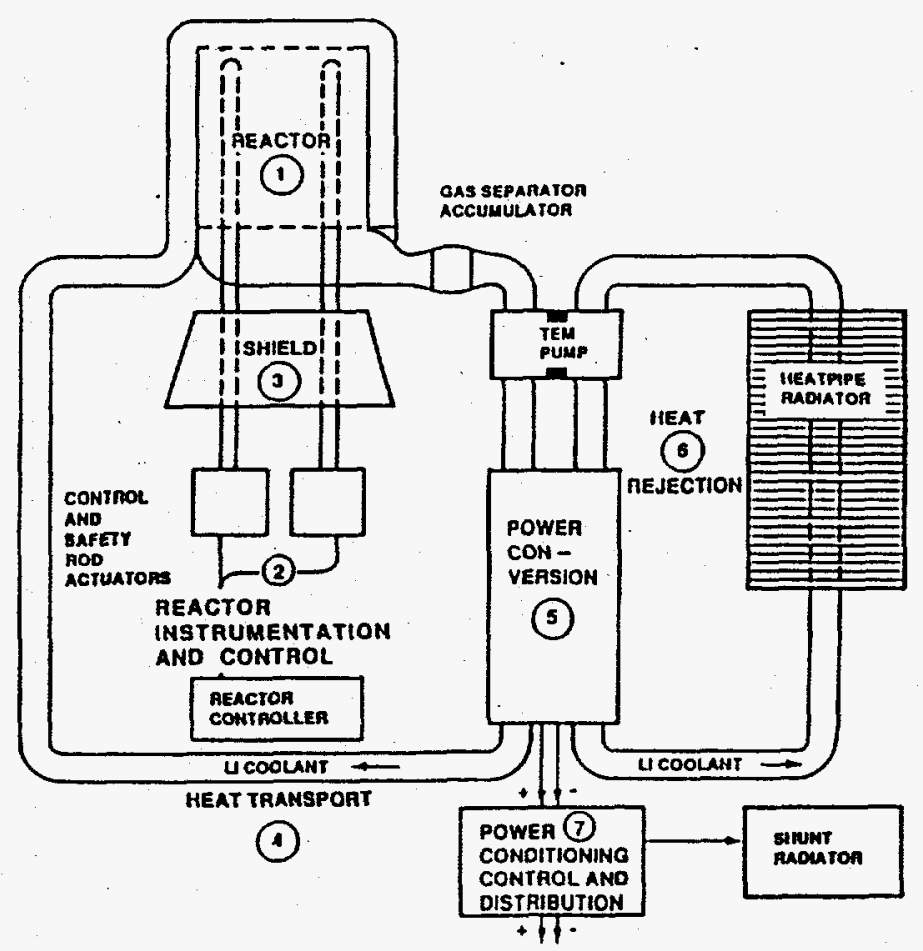

Figure 4-1.1 SP-100 Subsystems

The estimated total mass is $3600 \mathrm{~kg}$ for the $100 \mathrm{~kW}$ output system with a design lifetime of 10 years of which the reactor is operating for 7 years at full thermal power output. This GFS design configuration was adapted for outer planet NEP science missions by retaining all design elements except the auxiliary 
cooling loop function (intended for Earth orbiting missions) for mitigating an unlikely loss of coolant accidents. The end-of-mission reactor coolant outlet temperature was allowed to increase to $1400^{\circ} \mathrm{K}, 25^{\circ} \mathrm{K}$ above the GFS $1375^{\circ} \mathrm{K}$ design value. Certain other adjustments were also introduced which in aggregate produced an estimated total mass to $3930 \mathrm{~kg}$ for a system with a 14 year lifetime and 10 years of full power operation. Note that the mass distribution represented in figure 4-1.2 does not contain these design adjustments.

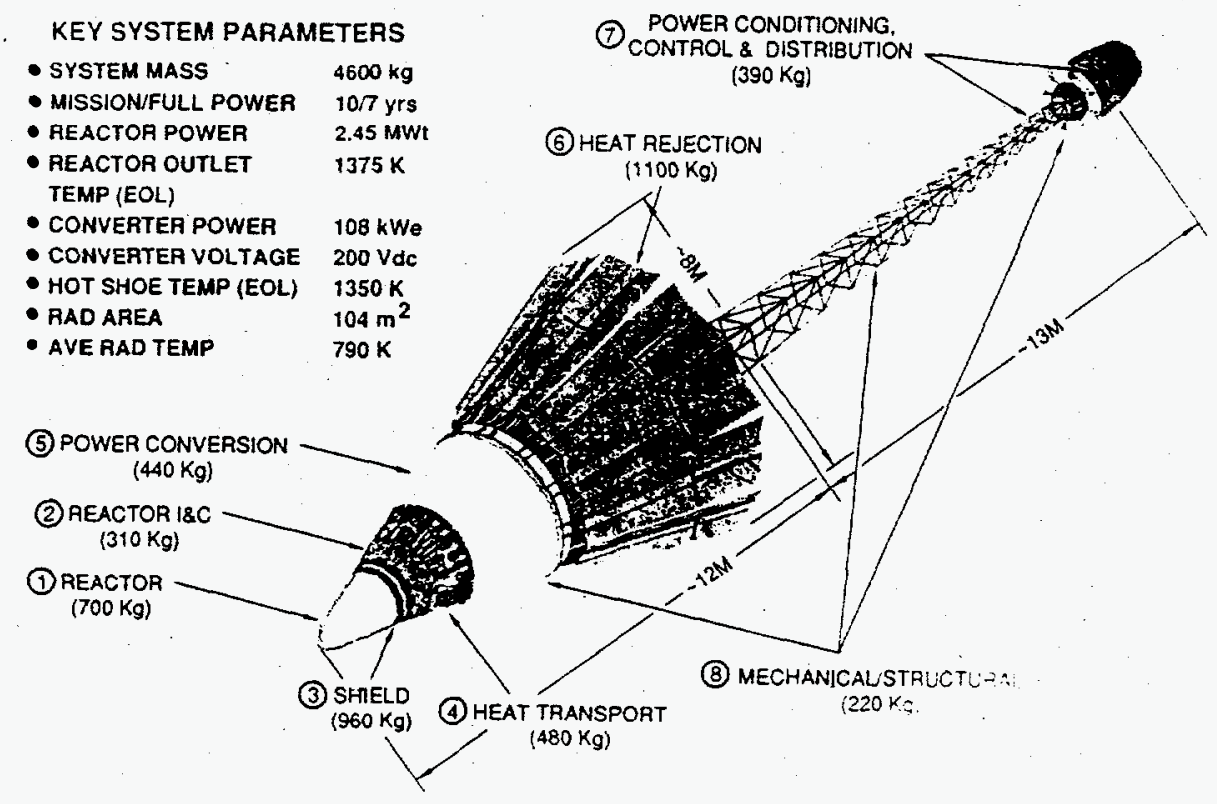

Figure 4-1.2 SP-100 GSF Design

\subsubsection{Power Plant Mass vs. Power and Life}

The key parameter of a power plant relevant to the analysis of a mission performance is the mass. Other important parameters are the SRPS full-power time needed for thrusting and the overall lifetime at least equal to the duration of the mission.

Figure 4-2 gives the predicted power system mass as a function of the power levels for two pairs of (full-power, life-time) cases. Linear equations representing the curves are also shown in the figure. Interpolation or extrapolation from these two curves is used to calculate the masses for other full-power time points.

Other ongoing technology development programs outside of the SP-100 program could provide opportunities for further mass reduction of the SP-100 design concept. Notably, advances in thermoelectric materials and in the waste heat and radiator designs could significantly reduce the total system mass. However, their technology development timeline is uncertain. These sources for mass reduction were therefore not considered in the current study.

\subsubsection{Radiation}

At the user plane $25 \mathrm{~m}$ away from the reactor, a neutron fluence of $10^{13} 1-\mathrm{MeV}$ equivalent is predicted for a ten year mission as illustrated above in Figure 4-3. The corresponding gamma dose is approximately $5 \times 10^{5} \mathrm{Rad} \mathrm{Si}$. For NEP missions considered here the full-power operation period will be two to three times longer and proportionately greater radiation exposure is implied. However, a quick study on the radiation 
shielding effect of the EP propulsion system made by Dr. Manvi (JPL) indicated that the EP propulsion system can have approximately a factor of ten and three effectiveness in reducing the neutron fluence and gamma dose respectively if it is placed between the spacecraft and the reactor. If the GFS shield design and user plane separation satisfy the generally accepted radiation tolerance limits $\left(10^{13} \mathrm{~N}\right.$, and $5 \times 10^{5}$ Rad Si), it is anticipated that the same holds true in NEP planetary mission applications. Naturally, spot shielding or use of rad-hardened parts or clever packaging will be needed to assure the survival of the sensitive electronics and sensors on board.

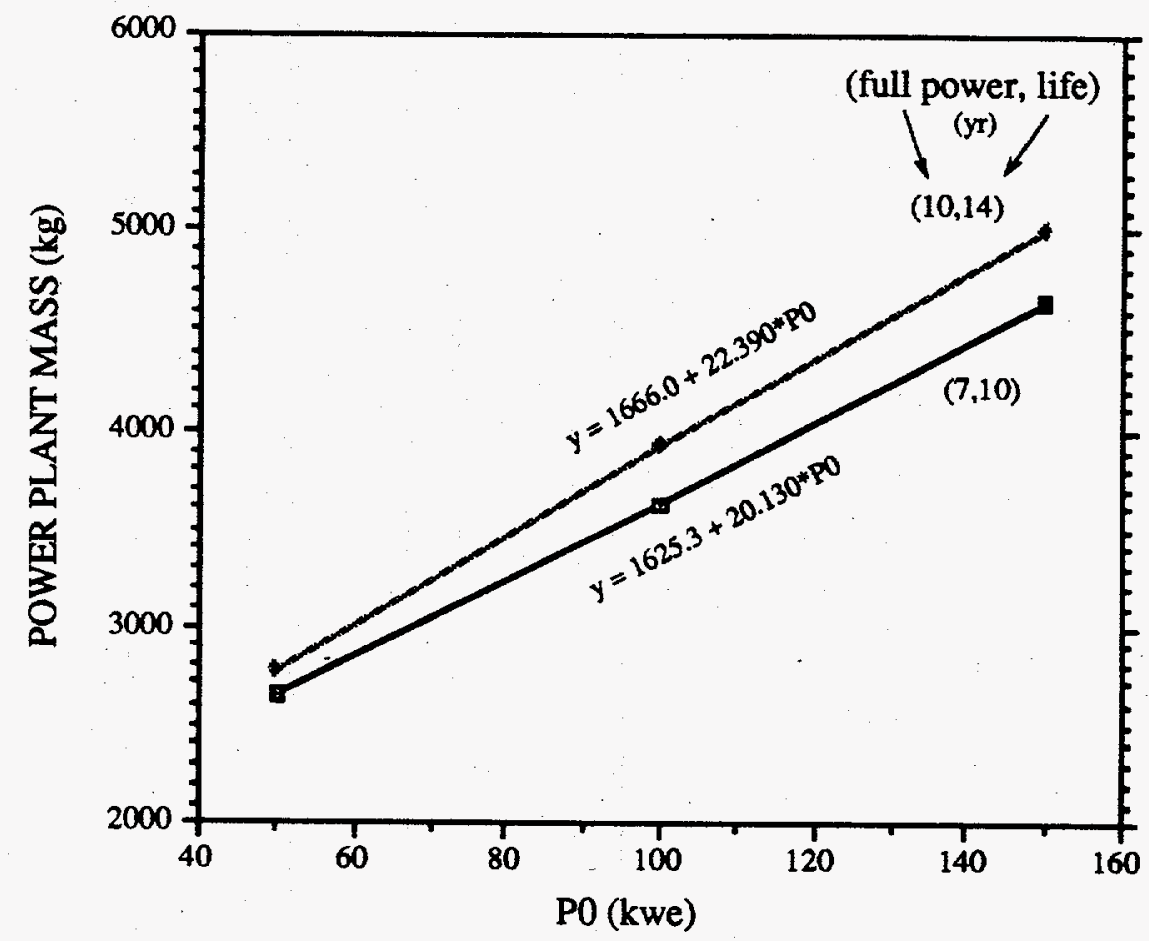

Figure 4-2. Space Nuclear Power System Mass

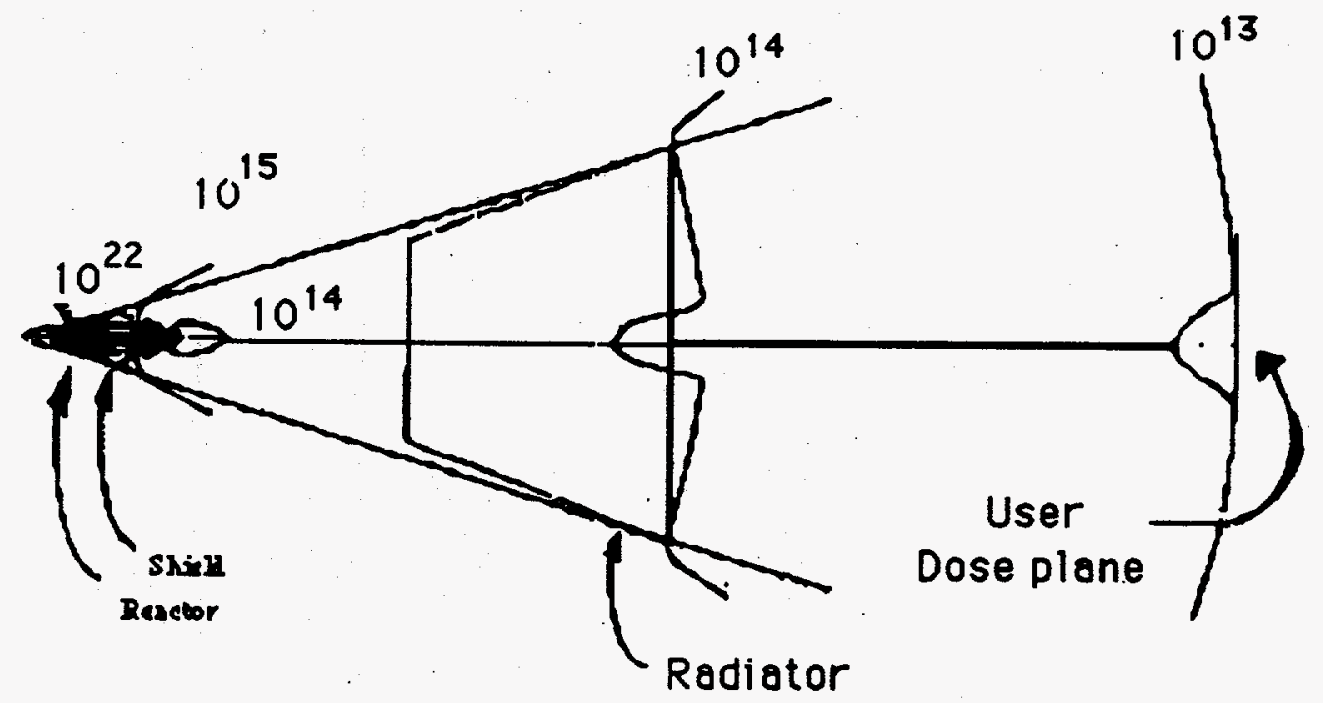

Figure 4-3. Neutron Fluence (1-MeV Equivalent) Profiles 


\subsection{Thrust Subsystems}

\subsubsection{Ion Engine Description}

An ion engine consists of a cylindrically shaped discharge chamber and a set of outwardly-dished molybdenum grids containing over 15,000 individual holes 1.3 to $1.9 \mathrm{~mm}$ in diameter. Electrons emitted by the cathode and accelerated towards the anode collide with neutral gas atoms injected into the discharge chamber and ionize them, forming a low voltage plasma that is maintained by the anode-cathode discharge of 30 volts, $30 \mathrm{amps}$. An axially-diverging magnetic field inhibits electron flow to the anode and increases the total efficiency of the engine. Ions in these plasma are then extracted out of the ion engine by applying an appropriate voltage to the screen-accelerator grids (e.g. 1100 and -500 volts respectively). A neutralizer, an electron-emitting cathode, maintains charge neutrality. When the plasma density, electron temperature, and screen-accelerator grid voltages are at certain values, the plasma ions exit through the holes in the screen and accelerator grids at velocities approaching 30 to $100 \mathrm{~km} / \mathrm{s}$ depending on the design. These exhaust velocities are $\sim 10$ to 30 times greater than those attained by burning chemical propellants. Therefore, the total thrust imparted per kilogram of fuel is 10 to 30 times larger in ion engines, compared to chemical rockets.

\subsubsection{Developmental Status}

Research in ion propulsion has been ongoing since the early 1960s. The SERT II Flight Program ${ }^{14}$ for the space testing of thrusters was executed in 1970 but failed after 3800 hours of operation. The SEPS development program in the early 1980's focused on the development of $30 \mathrm{~cm}$ mercury thrusters (J-series) operating at $2.7 \mathrm{~kW}$, specific impulse of 3000 seconds and a design operating life of 15000 hours. The thruster, successfully life tested for over $\mathbf{4 0 0 0}$ hours, and operated with an efficiency approaching $70 \%$. The life-limiting mechanism was found to be due to erosion of the baffle and pole pieces.

Since 1981, inert gases argon, krypton and xenon have replaced mercury as the propellants of choice. The switch was prompted by concerns over spacecraft contamination, system complexity and by the fact that inert gas ion thrusters are easier to ground test. Considering the large amounts of propellant (up to $5000 \mathrm{~kg}$ ) required for the missions, the availability and the cost, krypton is regarded as the logical choice. Cryogenic storage of the propellant is necessary in order to restrict the mass of the propellant tank (tankage fraction of $\sim 6 \%$ vs. $25 \%$ ) and to make room for the payload at the end of a mission.

Since 1982, Jet Propulsion Laboratory (JPL) and NASA Lewis Research Center (LeRC) have been designing and testing high power ( 5 to $10 \mathrm{~kW}$ ) inert gas thrusters at specific impulses greater than 4000 seconds ${ }^{28-30}$. The 30-cm ring-cusp thrusters, suggested for NEP science mission applications, have been tested and found to perform with good efficiencies $(68 \%)$. A ring-cusp thruster uses rings of magnets to form a cusp magnetic field geometry and allows the operation of thrusters without the baffle and pole pieces, thus improving operating life over that of the J-series design. Various design modifications and techniques to deal with other life limiting factors; erosion of accelerator grid and cathode starter electrode; are underway at these laboratories.

The development of ion engines in the past thirty years have achieved necessary performance levels (thrust, specific impulse and efficiency) for planetary missions but the predicted engine life has never been successfully demonstrated. A demonstration of the engine life required for a planetary mission would take 3 to 4 years and be extremely costly. Recently, there has been a breakthrough at JPL that enables ion engines to be life-tested at vacuum chamber pressures an order of magnitude higher than previously possible (3e-5 torr vs. $3 \mathrm{e}-6$ torr), greatly reducing the cost of testing. This is made possible by the addition of an electrode to the ion accelerator system which suppresses the adverse effects of testing at high background pressures without degrading the engine performance. This makes it possible to life-test in modest vacuum chambers with modest pumping systems, with a net savings of several million dollars. 
An overview of EP technology readiness status is shown in figure $4-4$.

- Engine and Autonomous Computer Control systems require moderate development

- Power processor, System Thermal Control and power source not yet developed

- Propellant Systems and Gimbals can use flight qualified hardware

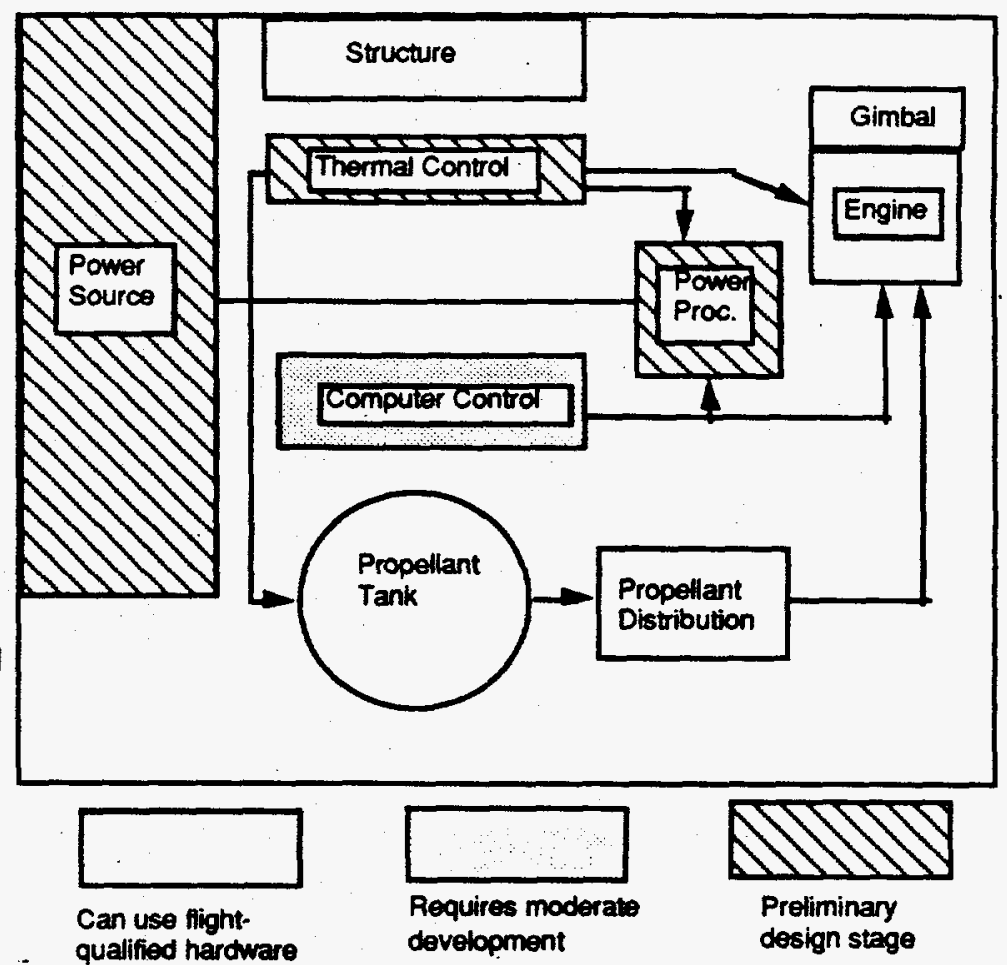

Figure 4-4 Ion Propulsion System Technology Status

\subsubsection{Thruster Performahce Characteristics}

Conservatively projected performance of the $30-\mathrm{cm}$ ring-cusp thruster as a function of specific impulse is shown in Table 4-1 with krypton as the propellant. The total efficiency depicted in Table 4 includes the power processor unit (PPU) operating at $93 \%$ efficiency. The projected lifetime of thrusters under deep space conditions is 10,000 hours.

Table 4-1 30-cm Ring-cusp Thruster Performance Characteristics

$\begin{array}{ccc}\text { ISP (sec) } & \begin{array}{c}\text { Power Input to } \\ \text { PPU (kw) }\end{array} & \begin{array}{c}\text { Total Efficiency } \\ (\%)\end{array} \\ 5000 & 8.3 & 64 \\ 6000 & 10.0 & 67 \\ 7000 & 11.7 & 68 \\ 8000 & 13.3 & 69 \\ 9000 & 15.0 & 70 \\ 10000 & 16.7 & 70\end{array}$

\subsubsection{Thrust Subsystem Architecture}

For high power NEP mission applications, a cluster of $30 \mathrm{~cm}$ thrusters (see Figure 4-4) configured to function as one unit from a single processor unit has been proposed by Brophy 31 . The functionality of this engine is indistinguishable from that of a more conventional engine configuration except that there are multiple hollow cathodes to be controlled. 
The number of thrusters clustered to operate as a unit is varied according to the specific impulse and power level required for the mission. The number of cluster units installed is determined by the total thrust burn time requirement of the mission and the level of redundancy desired. Since no useful reliability data exists, the redundancy is provided arbitrarily in the form of a $25 \%$ margin in thruster operating life.

As a ground rule for the analysis, the installation of two PPUs, the second as a standby, is assumed. This is regarded as appropriate because the predicted operating life of the PPU is much longer (5-15 years) than the thruster.

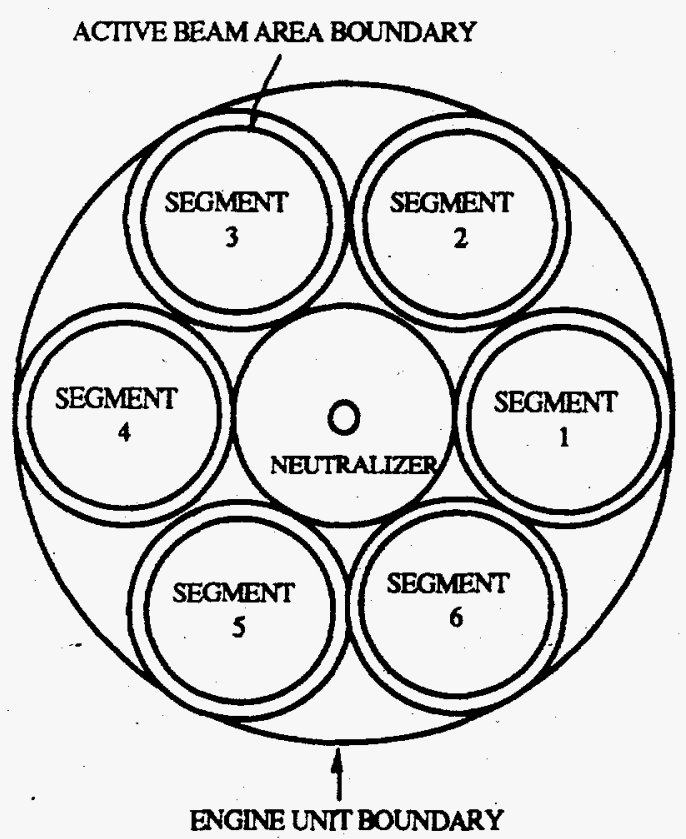

Figure 4-4. Example Segmented Engine Configuration

\subsubsection{Thrust Subsystem Mass Characteristics}

Mission performance is intimately tied to the mass efficiency of the propulsion systems. To facilitate the performance analysis the following thrust subsystem mass scaling equation is used for optimization of the NEP system parameters.

$$
\mathrm{M}_{\mathrm{THS}}=1.03(8.3 \mathrm{Nc} \mathrm{N} \mathrm{th}+4 \mathrm{NPPU} \mathrm{POPPU}+50+\mathrm{MP}+0.06 \mathrm{MP})
$$

where:

MTHS = total thrust subsystem mass in $\mathbf{k g}$.

POPPU = power input to PPU, in $\mathrm{kW}$.

$\mathrm{Nc}=$ no. of engine clusters

$\mathrm{Nth}=$ no. of ion-sources per cluster

NPPU = no. of PPU installed

$\mathrm{M}_{\mathrm{P}}=$ propellant mass in $\mathbf{k g}$.

The first term within the bracket accounts for the thruster mass including the support mass for clustering. The second term accounts for the contribution from the PPUs. The estimated mass for the PPU is $4.0 \mathrm{~kg} / \mathrm{kw}$ which includes the allowance for switching devices to provide multi-pronged thruster connections. The third term represents the computer control subsystem for the propulsion'system and the last two terms are the propellant and the tankage mass. The assumed propellant is Krypton. Although xenon offers higher efficiency, availability and cost considerations associated with the required large quantity 
(many thousands $\mathrm{kg}$ ) dictated this choice. The cryogenic propellant tank mass fraction is estimated to be about $6 \%$.

The multiplication factor 1.03 indicates that the structural support mass is $3 \%$ of the main thrust subsystem.

\subsection{NEP MISSION PERFORMANCE ANALYSIS}

\subsection{Approach}

One useful performance analysis conducted in the past examined the capability of one specific NEP propulsion system for various missions, including some studied here. The bulk of assumptions made with regard to both the SP-100 and thruster performance at the time were more optimistic than those presented in this study. Judging from the results of that study, it was felt that a single point design examination would probably not reveal fully the performance potential of the NEP. So, steps were taken in this study to explore the maximum NEP capability by optimizing NEP and mission parameters as best one can for each mission studied.

\subsection{NEP Mission Performance Parameters}

\subsubsection{Launch Vehicles}

Currently, the largest NASA launch vehicle available for interplanetary travel is the upgraded Titan IV(SRMU)/Centaur capable of lifting about $9,500 \mathrm{~kg}$ to escape. Shuttle-C/Centaur with a lift capability of $-15,000 \mathrm{~kg}$ was under development for a 1995 delivery but the development program has since been canceled and NASA has initiated a NLS (National Launch System) development program instead. Although, the characteristics of the heavy launch vehicles under the NLS program are unknown, its capability is anticipated to equal or surpass Shuttle-C. The date of availability is projected to be in the late 1990 's. Since about year 2005 is the earliest date a NEP mission can be contemplated, its availability is quite probable. For lack of a better choice, the performance analysis considers Shuttle-C/Centaur capability as representative of a heavy launcher and is noted as HLV/Centaur in this report.

\subsubsection{Earth Escape Options}

Two options can be considered for a NEP spacecraft to escape Earth and to begin interplanetary travel. They are: 1) to use an all chemical launch to escape and to tum on the NEP power after the escape condition is achieved or 2) to use a combination of chemical and NEP to spiral out of earth orbit.

This study regards the first option to be a simpler and preferable option to be adopted if a mission can be conducted using currently available launch vehicles. Past NEP mission concepts advocated the second option in which chemical rockets are used to deliver a NEP spacecraft to an intermediate starting Earth orbit followed by a NEP spiral to complete the escape. From the standpoint of mass performance, the spiral escape is more advantageous because the specific impulse of EP is at least one order of magnitude higher compared to that of a chemical launch vehicle. However, there are several drawbacks: 1) the operation of a nuclear powered vehicle in earth orbit can be controversial and complicates mission operations; 2) adverse impacts of Van Allen radiation on the flight system health exist, 3) mass penalty due to 1) and 2) is implied; 4) 1 to 2 years is required for the escape, thus that much longer flight time and thrust time are implied.

Unlike the SEI class of missions, the payload of a science mission is modest and the ability to deliver a larger mass may not necessarily be the overriding factor in designing a mission. The approach taken in this study is that earth spiral escape will be considered only if it becomes necessary. 


\subsubsection{Trajectory Optimization}

The maximization of NEP mission performance is made by optimizing key NEP parameters. Power (P0) and specific impulse (ISP) are the two key NEP parameters considered. This optimization requires an equation representing the NEP system mass as a function of PO and ISP. The linear relationship of the SRPS mass to $P 0$ as shown by the equations in Figure 4-2 can readily be incorporated in the analysis. However, the thrust system mass dependence on power and ISP is not as simple. The number of thrusters and power processors required depend on the thrust time in addition to the performance data presented in Table 4-1 and the mass scaling equation. The authors, based on a priori knowledge of the missions, converted the thrust subsystem data to an approximate linear function and represented the overall NEP mass vs power as follows.

$\mathrm{M}_{\mathrm{NEP}}=\left[1700+40^{*} \mathrm{P0}\right](\mathrm{kg})$

This is used merely to size the near optimal NEP system, but not in the actual performance assessment. Actual synthesis of the NEP system is made based on the NEP system described in the previous section. More elaborate mass representation and refined parameter optimization can be devised when such an effort is warranted.

The JPL low thrust optimization code is adequately formulated to calculate the performance pertaining to the heliocentric portion of the mission. However, the same cannot be said about the formulation with respect to the planetary phase of the mission. Computer programs to simulate and optimize trajectory and thrust history in a planetary phase do not exist. Approximate $\Delta V$ requirements are calculated using the method of Edelbaum ${ }^{32}$ and are accounted for in the performance assessment. This account is made by converting the calculated total $\Delta V$ requirement into an equivalent final circular capture orbit requirement. By so doing, the thrust time requirement in the planetary phase as well as its implication on fuel requirement is generated using the spiral performance formulation of Melbourne et. al. ${ }^{33}$. Note that Table 3-8 shows the equivalent final orbit size for each of the proposed mission scenarios. This deficiency, although not serious from the performance assessment point of view, is unfortunate because the maneuverability at the target planet is touted as the main benefit of NEP application and yet there is no reliable tool to validate the concept.

\subsection{Mission Scenarios and Performance}

This section presents mission scenarios and performance features of both the ballistic and NEP options in a comparative mode. Use of both launch vehicles, Titan IV(SRMU)/ Centaur and Shuttle$\mathrm{C} /$ Centaur are considered in the performance comparison. Although the payload assumed is of the Mariner Mark II class, the parametric results presented should be useful in understanding the NEP potential even if other payload goals are considered.

Extensive data showing the NEP performance capability and the system requirements versus heliocentric flight times are provided in section 5.9 for all the missions studied. The purpose of this data display is to provide sufficient information necessary to understand the sensitivity of mission feasibility with respect to various NEP system component uncertainties.

\subsubsection{Pluto Orbiter/Probe}

\section{Ballistic Mission Concept}

Pluto is the last of the major planets yet to be explored with a spacecraft. NASA is currently interested in a reconnaissance mission arriving before the suspected collapse of the Pluto atmosphere. In recent years, two options for fast flybys have been proposed. The Pluto-350 option uses a 2001 EJ gravity assist trajectory (13-15 year trip) and delivers a $350 \mathrm{~kg}$ spacecraft to briefly encounter the Pluto-Charon system at a flyby speed of about $13 \mathrm{~km} / \mathrm{s}$. A second option, delivers a $<100 \mathrm{~kg}$ microspacecraft in 7-10 years using a Titan IV(SRMU)/Centaur with two additional Star 48 kick motors. The flyby speed is 25 
$\mathrm{km} / \mathrm{s}$. Because of the weak gravity of Pluto to aid in orbit capture, an orbiter mission is extremely difficult to implement using a ballistic mode unless a very long flight time, approaching $\mathbf{4 0}$ years, is acceptable.

\section{NEP Mission Concept}

In contrast to the chemical mode, the very weak gravitational pull of Pluto is an attractive feature for the NEP mission design. Due to the effectiveness of NEP propulsion far away from the Sun, it is possible for the spacecraft to arrive at Pluto with a $V_{\infty}$ of 0 , thus a rendezvous state is nearly achieved and will require only a modest amount of $\Delta V$ thereafter to explore both Pluto and Charon. A rendezvous mission with Charon comes first before the spacecraft spirals into a tight orbit about Pluto. Performance permitting, a lander may be deployed at both bodies. A lander can provide in-situ measurements of the surface as well as the atmosphere during its descent.

\section{NEP Performance Summary}

Results of the performance analysis are presented in Tables 5-1.1 and 5-1.2. The performance capability and NEP system requirements versus heliocentric flight times are provided. The performance achieved using both launch vehicles, a Titan IV(SRMU)/Centaur and a HLV/Centaur, are shown. Summary notes commenting on the results are appended to the table for easier comprehension.

Performance Using Titan IV(SRMU)/Centair + NEP: An orbiter mission to Pluto and Charon (no probes) is feasible with a 14.5 year trip. The sensitivity of the performance is roughly $300 \mathrm{~kg}$ per year of trip time. The dry NEP system (@ P0 $60 \mathrm{~kW}$, and ISP $\approx 400 \mathrm{sec}$.) weighs $\sim 4000 \mathrm{~kg}$. The mission may tolerate $\sim 15 \%$ of NEP system mass uncertainty, if the flight time is increased to 16.5 years (trip time longer than 15 years is probably unsatisfactory). If the mass growth of the total flight system can be controlled, after considering the impacts of all potential complications anticipated in a NEP mission, this may be an early NEP mission candidate.

The reasons for an early application of NEP on PLO are: 1) the science content of the mission is greatly improved over the ballistic counterpart, orbiter vs fast flyby; 2 ) it appears to be feasible with the currently available launch vehicle; 3 ) the trip time is comparable to the ballistic flyby mission (13-15 years) and not too objectionable; and 4) there is a need for arriving at Pluto before 2020-2025.

Performance Using HLV/Centaur + NEP An 11.5-year orbiter (no probes) mission is feasible. An additional year of flight time allows the delivery of a $414 \mathrm{~kg}$ lander to Pluto or Charon. With a 13.5 year flight a lander on both can be accommodated. A reasonable performance margin exists.

\section{NEP Earth-Pluto Heliocentric Trajectory}

An example heliocentric transfer trajectory (ecliptic projection) is shown in Figure 5-1. A "thrust coast - thrust" is the common thrust profile needed for rendezvous and $v_{\infty}=0$ planetary encounter trajectories. Due to the low level of NEP thrust and the near zero earth escape energy, the spacecraft begins with a spiral about the sun for a while until it gathers enough energy to head towards the outer planets.

\section{NEP - Ballistic Performance Comparison}

Figure 5-2 presents the payload mass delivered as a function of heliocentric transfer time. The figure illustrates clearly the superiority of NEP over chemical propulsion irrespective of the payload requirements, and implies that no significant exploration of Pluto can be made without NEP. 


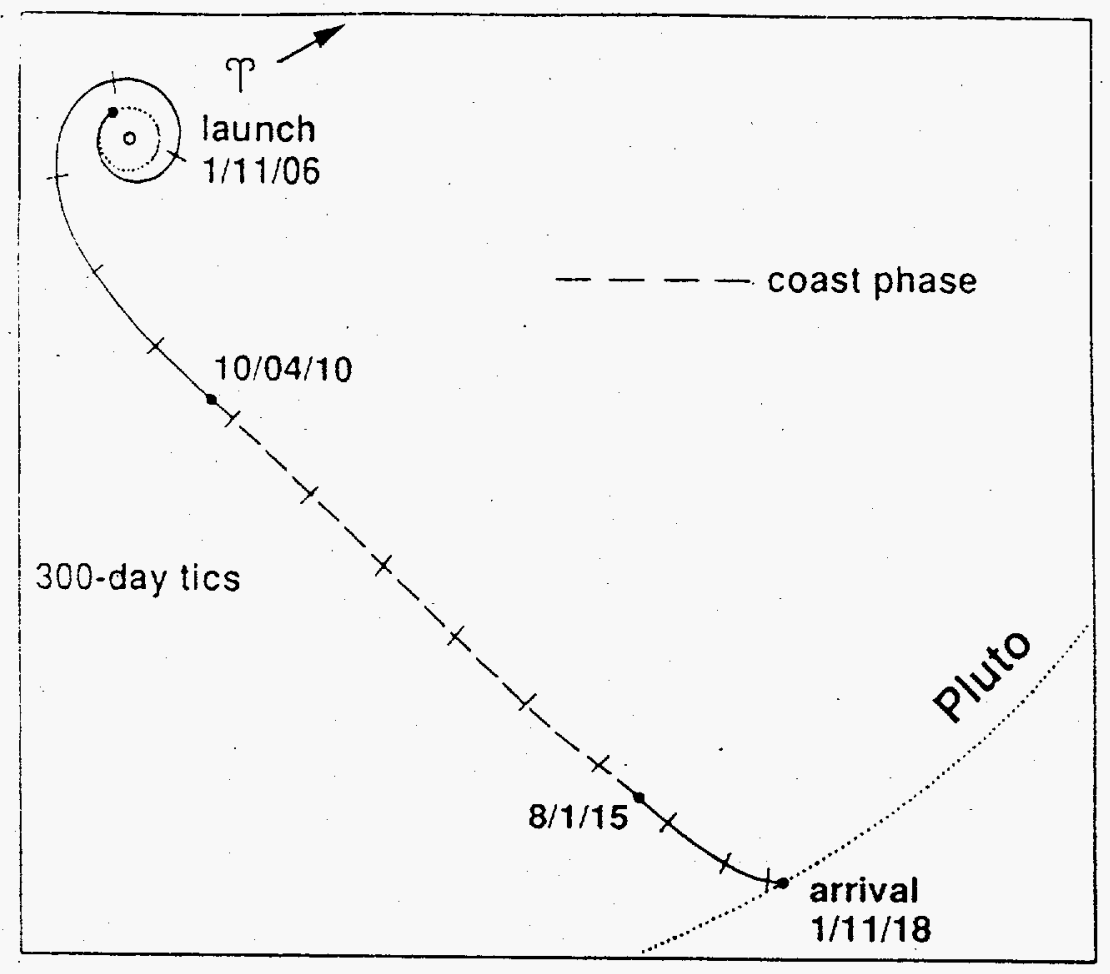

Figure 5-1 2006 Earth-Pluto Trajectory

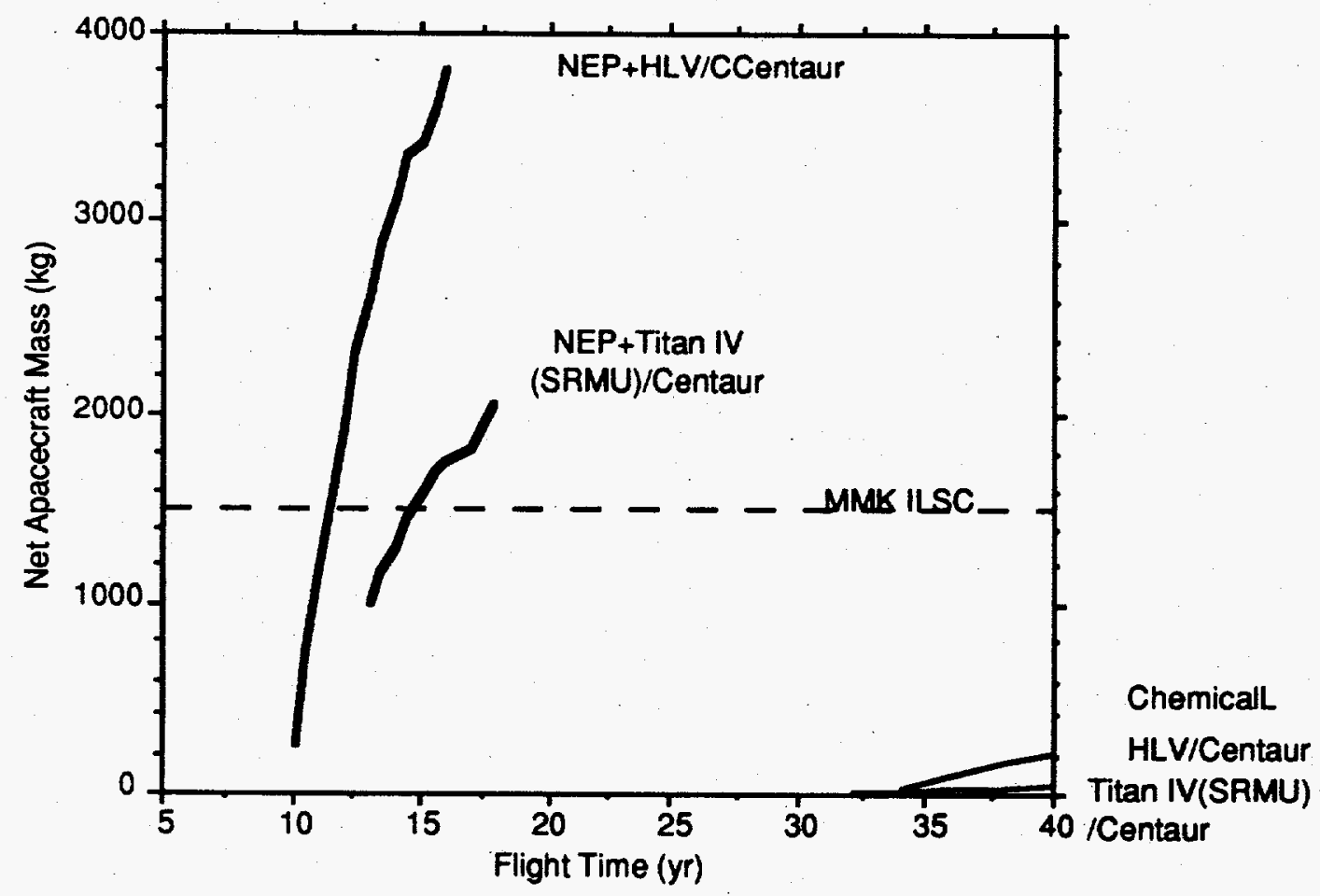

Figure 5-2 Pluto Orbiter Performance Comparison 


\subsubsection{Neptune Orbiter/Probe}

\section{Ballistic Mission Concept}

A 2002 launch with a multiple Venus, Earth and Jupiter (VVEJ) gravity assist trajectory, was considered for this mission to deliver the required mass. The main drawback attributed to this chemical mission option is the long flight time; $\geq 18$ years. The orbital design at Neptune is also severely restricted by the on-board $\Delta V$ capability of a Mariner Mark II (MMK II) spacecraft. Like Galileo and Cassini, the orbital sequences are built around satellite (Triton) gravity assists to save $\Delta \mathrm{V}$ and acquire Triton science at each of the many $(\approx 45)$ swingbys. The flyby speed at Triton is 4 to $5 \mathrm{~km} / \mathrm{s}$. The viable mission opportunities are very few because the occurrence of a favorable planetary (Earth, Venus and Jupiter) alignment is rare. Typically, a good launch opportunity involving a Jupiter swingby is available for about two consecutive years, but after that, a favorable Jupiter-Neptune alignment will not recur for about another 13 years.

\section{NEP Mission Concept}

The proposed NEP mission consists of the delivery of a spacecraft to orbit about Neptune and Triton, the delivery of a probe into the planet's atmosphere and fast flybys of Neptune's small satellites and ring systems. If a sufficient performance margin exists, the option of a Triton lander may be considered.

The NEP spacecraft is targeted in a posigrade manner to a low periapsis altitude after deploying the atmospheric probe on Neptune approach. The spacecraft does the probe relay link near periapsis and implements subsequent orbital maneuvers, including: posigrade to retrograde orbit change; Triton rendezvous; spiral into and out of $500 \mathrm{~km}$ orbit at Triton; and an inward spiral toward the ring zone for ring science and close range observations of Neptune. Along the way, flyby encounters with other satellites will be made. Unfortunately, due to the retrograde motion of Triton, this scenario is rather awkward and the encounters of minor satellites and rings will be fast flybys.

A more satisfactory scenario would be to deliver a separate Triton Orbiter equipped with its own chemical rocket at Neptune appioach so that the NEP spacecraft can spiral inward in a posigrade manner to rendezvous with other satellites and rings as well as delivering the Neptune probe. This is possible in principle with a NEP but would perhaps require an earth spiral escape and a higher power than the $100 \mathrm{~kW}$ class NEP.

\section{NEP Performance Summary}

Tables 5-2.1 and 5-2.2 summarize the NEP performance capability as a function of flight time and provides the associated mission parameters for this mission. The performance potential is summarized as follows.

Performance Using Titan IV(SRMU)/Centaur + NEP: An orbiter mission to Neptune using Titan IV(SRMU)/Centaur takes 17 years and shows a small improvement of performance with flight time. No comfortable performance margin exists and the flight time is not sufficiently better than the ballistic option to be attractive.

Performance Using HLV/Centaur + NEP A $1400 \mathrm{~kg}$ orbiter, with a $376 \mathrm{~kg}$ atmospheric probe and a small $(50 \mathrm{~kg})$ Triton lander can be delivered to Neptune in 12 years. With a larger $400 \mathrm{~kg}$ lander, a flight time of 13.5 years is needed. The performance sensitivity is $\sim 600 \mathrm{~kg}$ per year of trip time. The dry NEP system mass is about $6000 \mathrm{~kg}$ (@ P0 $100 \mathrm{~kW}$, ISP $=8000-10000$ sec.) The mission is totally feasible and offers a significant improvement over the ballistic mode in science and flight time.

\section{NEP Earth-Neptune Heliocentric Trajectory}

An example heliocentric transfer trajectory (ecliptic projection) is shown in Figure 5-3 


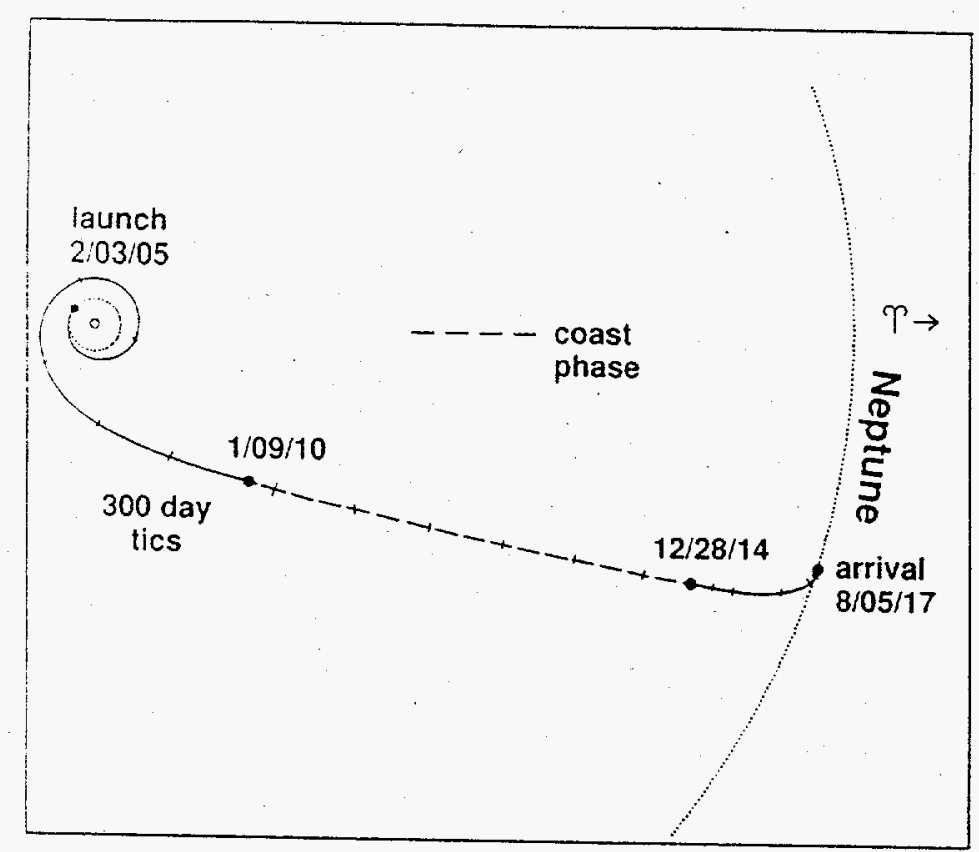

Figure 5-3 2005 Earth-Neptune Trajectory

\section{NEP - Ballistic Performance Comparison}

Figure 5-4 presents the performance comparison of NEP and chemical options.

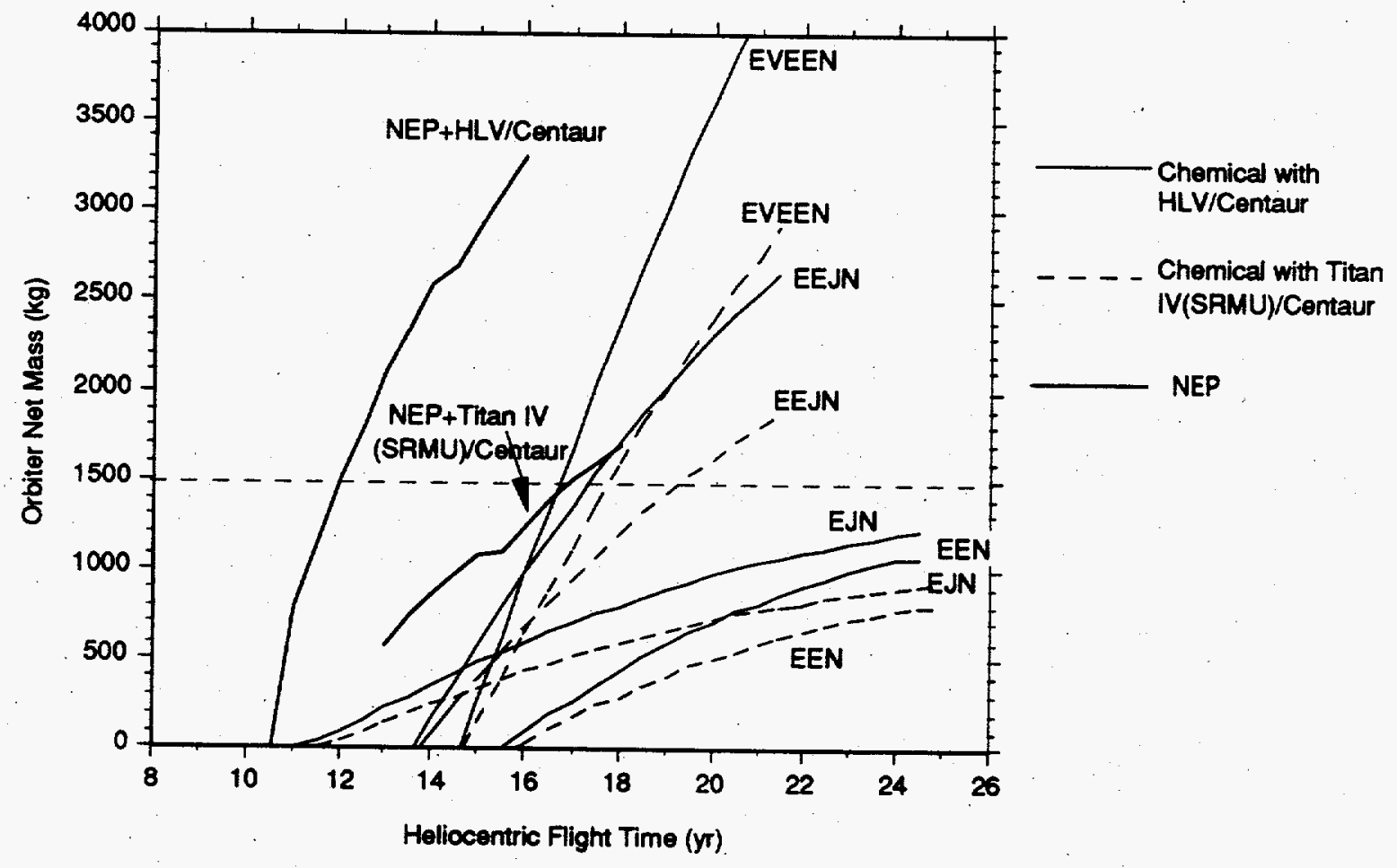

Figure 5-4 Neptune Orbiter/Probe Performance Comparison 
A fairly comprehensive set of gravity assist trajectories (e.g. EEJN, where the first letter stands for launch planet and the last letter denotes the arrival planet) are included for the latter. Due to the differences in the attainable mission scenarios between the two, no fair comparison is possible. This comparison, intentionally giving the chemical option many benefits of doubts, is made in order that the upperbound possibility of chemical option be compared to the NEP option. These benefits include assumptions such as perfect planetary alignment (which never occurs) and much less demanding mission scenarios at the target. The example injected orbit for ballistic option is 3 by $100 R_{N}$, with a navigational allowance of $500 \mathrm{~m} / \mathrm{s}$ for the total mission which is known to be very optimistic. By comparison the NEP scenario is much more ambitious as described in the previous paragraph.

The figure shows clear NEP flight time advantage. Even under the most optimistic assumptions, the ballistic flight time for NEO/P is $>16$ years using HLV/Centaur. It also shows that trajectories with a Jupiter flyby are the only viable ones and that the scarcity of launch opportunities (about two consecutive years for every 13 years, the Jupiter-Neptune synodic period) is implied. NEP flight can be made once every year.

\subsubsection{Uranus Orbiter/Probe}

\section{Ballistic Mission Concept}

Only a very preliminary ballistic mission concept 22 exists for this mission. The reference ballistic mission considers an Earth-Jupiter gravity assist trajectory and takes 15 years to reach Uranus. After delivering a Uranus probe the spacecraft makes all observations from a loose elliptic orbit. The limited onboard $\Delta \mathrm{V}$ capability and the absence of a useful gravity assist satellite dictates this observational condition. The science return is restricted mostly to distant flybys of satellites and observations of atmosphere and rings. Again, the mission dependence on Jupiter gravity assists results in sparse mission opportunities.

\section{NEP Mission Concept}

The NEP mission scenario differs from the case of NEO/P in that no major satellite (such as Triton) of interest is in retrograde motion to complicate the scenario. Upon arrival, the NEP spacecraft spirals gradually into the vicinity of the planet to about five Uranus radii. In the process, the NEP spacecraft releases an atmospheric probe, performs the relay link and subsequently rendezvous with each of the outer five moons (which vary in radius from 320 to $1010 \mathrm{~km}$ ). The probe can be released from a loose orbit, perhaps from the orbit of Uranus's furthermost satellite, Oberon, at 23.4 Uranus radii. However, the probe cannot be propelled into the atmosphere at the time it is released, because that would prevent establishment of a relay link with the spacecraft. The probe has to remain in this orbit until the NEP spacecraft reduces its orbit and can be in view of the probe during its descent through the atmosphere. Therefore, the probe will carry a motor to start the deployment kick of about $2.3 \mathrm{~km} / \mathrm{s}$. In short, this scenario offers the grand tour of Uranus satellites and rings and delivers an atmospheric probe.

\section{NEP Performance Summary}

Tables 5-3.1 and 5-3.2 summarizes the NEP performance capability as a function of flight time and provides the associated NEP system parameters. Key observations made from the table are as follows.

Performance Using Titan IV(SRMU)/Centaur + NEP: NEP performance is unsatisfactory with this launch vehicle, partially because the mission scenarios prescribed are ambitious. However, with a large investment implied by a NEP one would not want to opt for a minimal orbiter scenario as assumed in the ballistic option.

Performance Using HLV/Centaur + NEP: This mission scenario, proposing a satellite grand tour, the delivery of an atmospheric probe, plus tight circular orbiter is feasible with a (HLV/Centaur + NEP) system. The mission can be performed satisfactorily in 10.5 to 14 years with an ample performance margin. The optimal power is at about $100 \mathrm{~kW}$ and the specific impulse at a 8000 seconds level. 


\section{NEP Earth-Uranus Heliocentric Trajectory}

An example heliocentric transfer trajectory (ecliptic projection) is shown in Figure 5-5

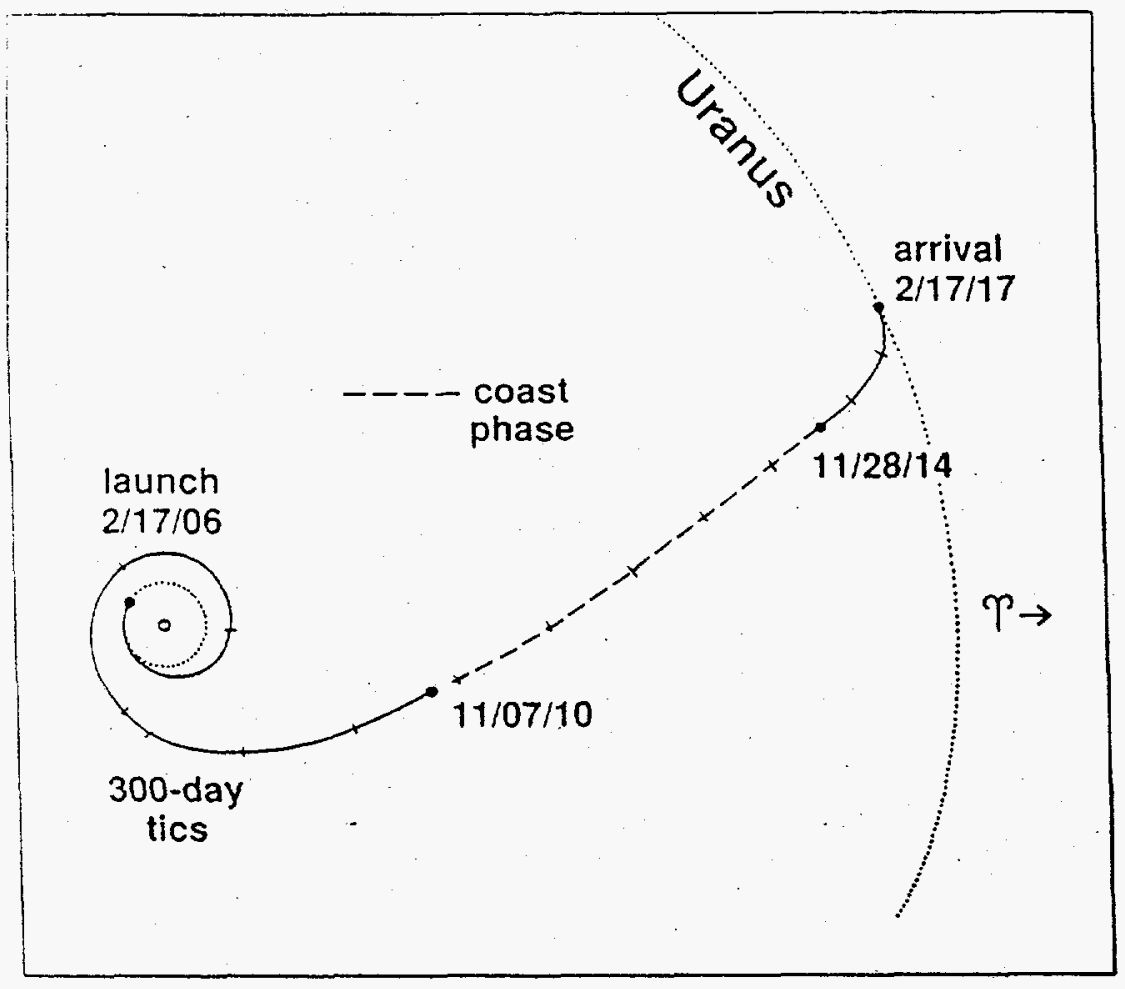

Figure 5-5 2006 Earh-Uranus Trajectory

\section{NEP - Ballistic Performance Comparison}

Figure 5-6 presents the performance comparison of NEP and chemical propulsion options. Various gravity assist trajectories are considered for the latter. On the surface NEP superiority is not very distinguished as in the case of Pluto or Neptune but one needs to bear in mind that a profound difference in science content between the two mission scenarios are involved as discussed above. The assumptions of perfect Venus, Earth and Jupiter alignment ; easy 3 by $100 R_{N}$ orbit capture; and small navigational allowance of $300 \mathrm{~m} / \mathrm{s}$ do illustrate the extent of comparative advantage accorded to ballistic option. The NEP scenario in essence is an ambitious grand tour of the Uranus planetary system.

The conclusion offered here is that, a minimal science mission of Uranus is possible with a 13 to 15 year flight time ballistically depending on whether one uses a Titan IV(SRMU)/Centaur or a HLV/Centaur but a comprehensive grand tour is achieved if NEP is mated with a HLV/Centaur. The performance of NEP using Titan IV(SRMU)/Centaur does not look attractive.

\subsubsection{Jupiter Grand Tour}

\section{Ballistic Mission Concept}

JGT is a NEP unique mission concept from its inception. It is possible to envision a ballistic mission with similar grand mission objectives but one quickly reaches the conclusion it is not possible to attain such a goal unless multiple launches are employed (see the performance comparison in the later section). 


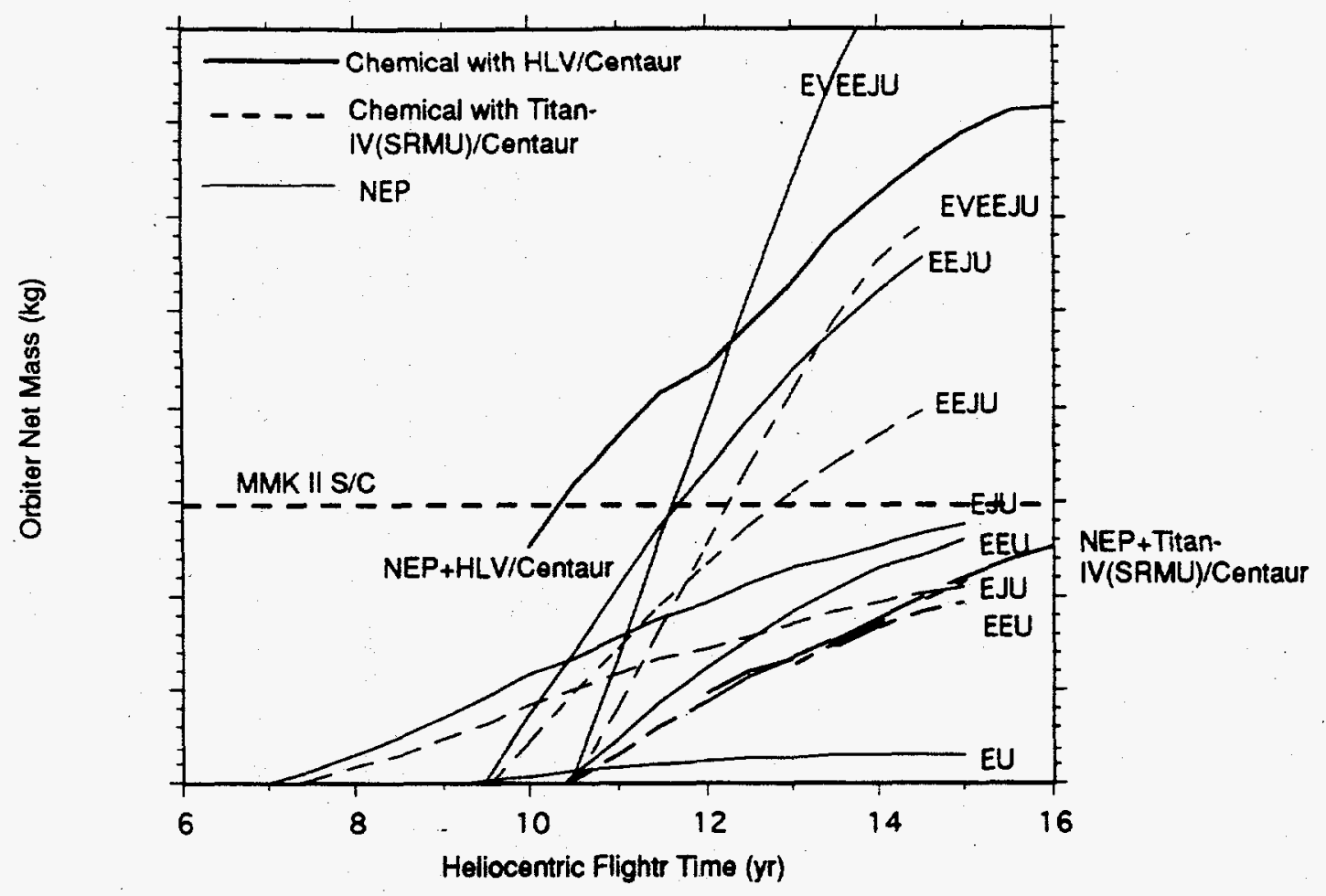

Figure.5-6 Uranus Orbiter/Probe Performance Comparison

\section{NEP Mission Concept}

Upon arrival at Jupiter $\left(v_{\infty}=0\right)$, the NEP driven spacecraft spirals in the Jovian gravity field to approach Callisto with zero $V_{\infty}$ which is followed by a spiral descent in the Callisto gravity field down to a $500 \mathrm{~km}$ circular altitude orbit. After accomplishing the Callisto science the spacecraft spirals out of the satellite sphere of influence and reenter the Jovian field for the next leg of the mission; to orbit about Ganymede; and then to Europa and IO. Encounters with other non-Galilean satellites are expected between these major encounters.

In cases where the performance exceeds the orbiter requirement, landers may be added or two JPO spacecraft (JPO-1 and JPO-2. with a total mass of $2500 \mathrm{~kg}$ ) 22 equipped with their respective chemical propulsion systems may be accommodated. They are to be injected into two different polar orbits on Jupiter approach before the spiral towards Callisto begins. JPO-1 which emphasizes Jupiter environment science is targeted to a final orbit of $1.014 \mathrm{RJ}$ by $20 \mathrm{RJ}$ polar orbit. JPO-2 is aimed at lo intensive investigations and is to be placed in a $5 R_{J}$ by $82 R J$ polar orbit.

\section{NEP Performance Summary}

Table 5-4.1 and 5-4.2 summarize the NEP performance capability as a function of flight time and provides the associated NEP system parameters. Key observations made from the table are as follows.

Performance Using Titan IV(SRMU)/Centaur + NEP: An orbiter class tour of all satellites (no probes) is feasible with a flight time of 5 to 6.5 years. This trip time is probably acceptable ( $\approx$ Galileo flight time), considering the significant amount of science return expected. The NEP system parameters are similar to 
those required for the PLO. The performance appears to peak at about a 7-year flight time, and the margin available is only about $10 \%$.

Performance Using HLV/Centaur + NEP: The orbiter class tour of all satellites is feasible with a flight time of 3.5 years. However, noting the rapid growth in the payload capability with the longer flight time, longer flight time options are recommended. The addition of two $1000 \mathrm{~kg}$ (includes the retro-motor) landers for two of the Galilean satellites or addition of two small fields and particles spacecraft $(2500 \mathrm{~kg}$ total ) are possible by choosing a longer 5-6 year trajectory.

\section{NEP Earth-Jupiter Heliocentric Trajectory}

An ex amplc heliocentric transfer trajectory (ecliptic projection) is shown in Figure 5-7.

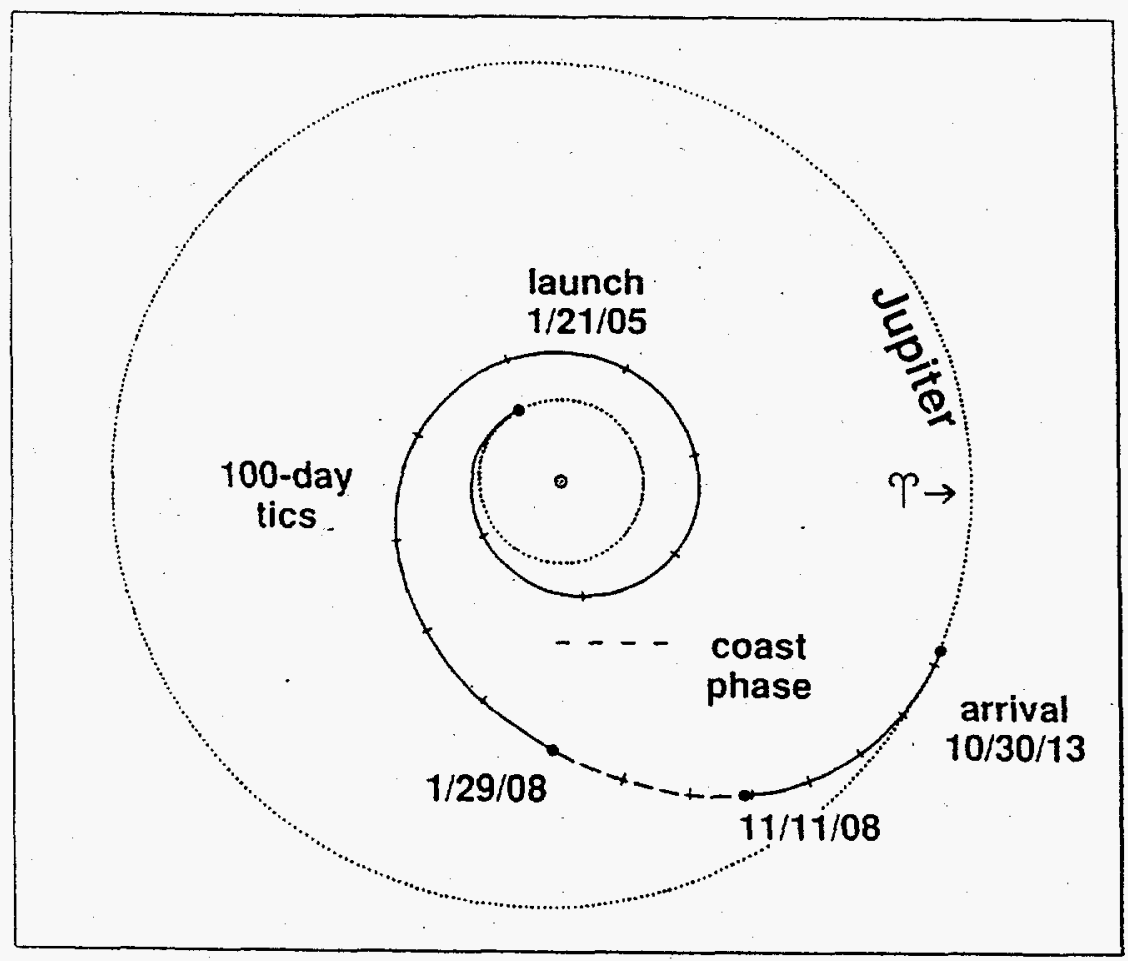

Figure 5-7 2005 Earth-Jupiter Trajectory

\section{NEP - Ballistic Performance Comparison}

Figure 5-8 presents the performance comparison of NEP and chemical options. Again various trajectory options are included for the ballistic option. The data points corresponding to ballistic options are for the most part for single-satellite orbiter missions. If a HLV/Centaur is used it may be possible ballistically to consider visiting two satellites, but only a small orbiter $(-200 \mathrm{~kg})$ can be delivered. Whether one uses HLV/Centaur or Titan IV(SRMU)/Centaur, a single satellite orbiter ballistic performance is far worse than the NEP capability of a four-satellite mission. There is no question as to the large advantages NEP offer in this mission.

A steep increase in NEP performance is seen when the flight time approaches 5 years, indicating a jump in the trajectory branch. With such a flight time, if a (NEP + HLV/Centaur) is available, the original grand concept of JGT including two JPO (fields and particles) spacecraft can be implemented. 


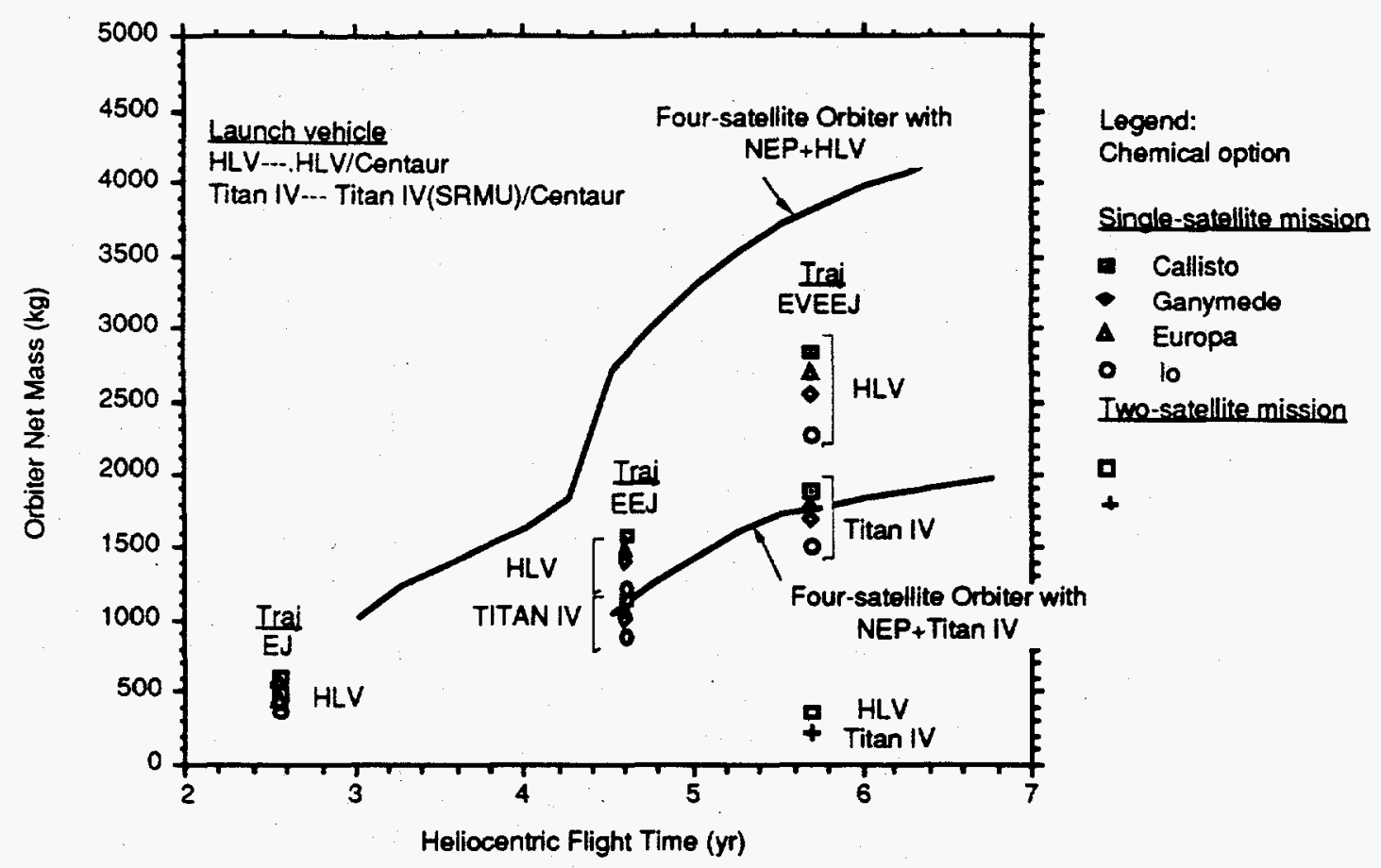

Figure 5-8 Jupiter Grand Tour Performance Comparison

\subsubsection{Multiple Mainbelt Asteroid Rendezvous}

\section{Ballistic Mission Concept}

To rendezvous with more than one asteroid within a reasonable time is difficult whether a Titan IV or $\mathrm{HLV}$ is used. The ballistic rendezvous mission needs to rely on Mars gravity assists to boost the mass performance. With a single Mars gravity assist, the flight time ranges from 2 to 5 years. This confines the accessibility to asteroids in the inner-belt $(-2-2.5 \mathrm{AU})$. In order to attain Mid-belt targets or to further reduce $\Delta \mathrm{V}$ requirements for the purpose of acquiring a second target, two Mars gravity assists are called for and extends the mission time to first target to 5 to 7 years.

\section{NEP Mission Concept}

The fuel efficiency of NEP offers ideal conditions for touring in the mainbelt. Once the spacecraft is delivered to the asteroid belt, there are opportunities to get to the next target without expending a large amount of fuel if a reasonable transfer time ( $\approx$ half the orbital period of asteroids) between asteroids is allocated.

Since Vesta and Ceres are two asteroids most frequently mentioned as desirable targets, an example MMBAR mission with a rendezvous sequence built around these asteroids was created to demonstrate the potential of NEP in performing this type of mission.

The proposed NEP mission concept consists of sending one orbiter spacecraft and optionally a number of landers or penetrators to orbit about six asteroids of prime interests. The spacecraft will observe an asteroid for a nominal duration ( $\approx 60$ days minimum) from a rendezvous state $(\approx 0$ relative velocity) and then proceeds to the next target.

\section{NEP Performance Summary}

Table 5-5.1 and 5-5.2 summarizes the performance of a typical MMBAR mission attainable with a NEP. Note that different target sequences are suggested here depending on the launch vehicle used. Although 
it is not necessary, the intent here was to illustrate the possibility of obtaining a shorter total mission time case when a HLV is given. Key observations made from the table are as follows.

Performance Using Titan IV(SRMU)/Centaur + NEP: This mission, involving six highly desirable targets (Amulthea-Vesta-Luthetia-Ceres-Leto-Eugenia), is within the reach of a (Titan IV(SRMU)/Centaur + NEP) system. Although only a small performance cushion is indicated in Table 5-5.1, there are a number of ways that are available to maintain the viability of this mission such as; change of targets, longer flight time or even reducing the number of targets. Note also that flybys of asteroids of opportunity are available to further enhance the science retum.

This is perhaps the best choice for the first NEP mission because it uses an available launch vehicle and for all the reason mentioned earlier.

Performance Using HLV/Centaur + NEP: Given a (HLV/Centaur + NEP), the anticipation at the outset of the analysis was that, MMBAR can be accomplished in a much shorter flight time than when a Titan IV(SRMU)/Centaur is used. Table 5-5.2 shows that the mission time is reduced by about two years but with no significant increase in payload margin. The indication is that shortening the flight time beyond the natural boundary or aiming for hard-to-access targets because they are more desirable are accompanied by a stiff performance penalty. The natural transfer time between asteroids is about half the orbital period of the asteroids, i.e. 1.5 years in the inner belt to 2.5 years in the mid-belt. On the average, 2 years per target is expected.

\section{NEP Multiple Mainbelt Asteroid Trajectory}

An example heliocentric transfer trajectory (ecliptic projection) of a MMBAR mission is shown in Figure 5-9. This is a thirteen year six target mission. Details of this example are given in Table 5-5.1.

Earth - Amulthea (SX,24km) - Vesta (V,288 km) - Luthetia (M, $54 \mathrm{~km})$ Ceres $(G, 516 \mathrm{~km})$ - Leto $(\mathrm{S}, 64 \mathrm{~km})$ - Eugenia(C, $122 \mathrm{~km})$

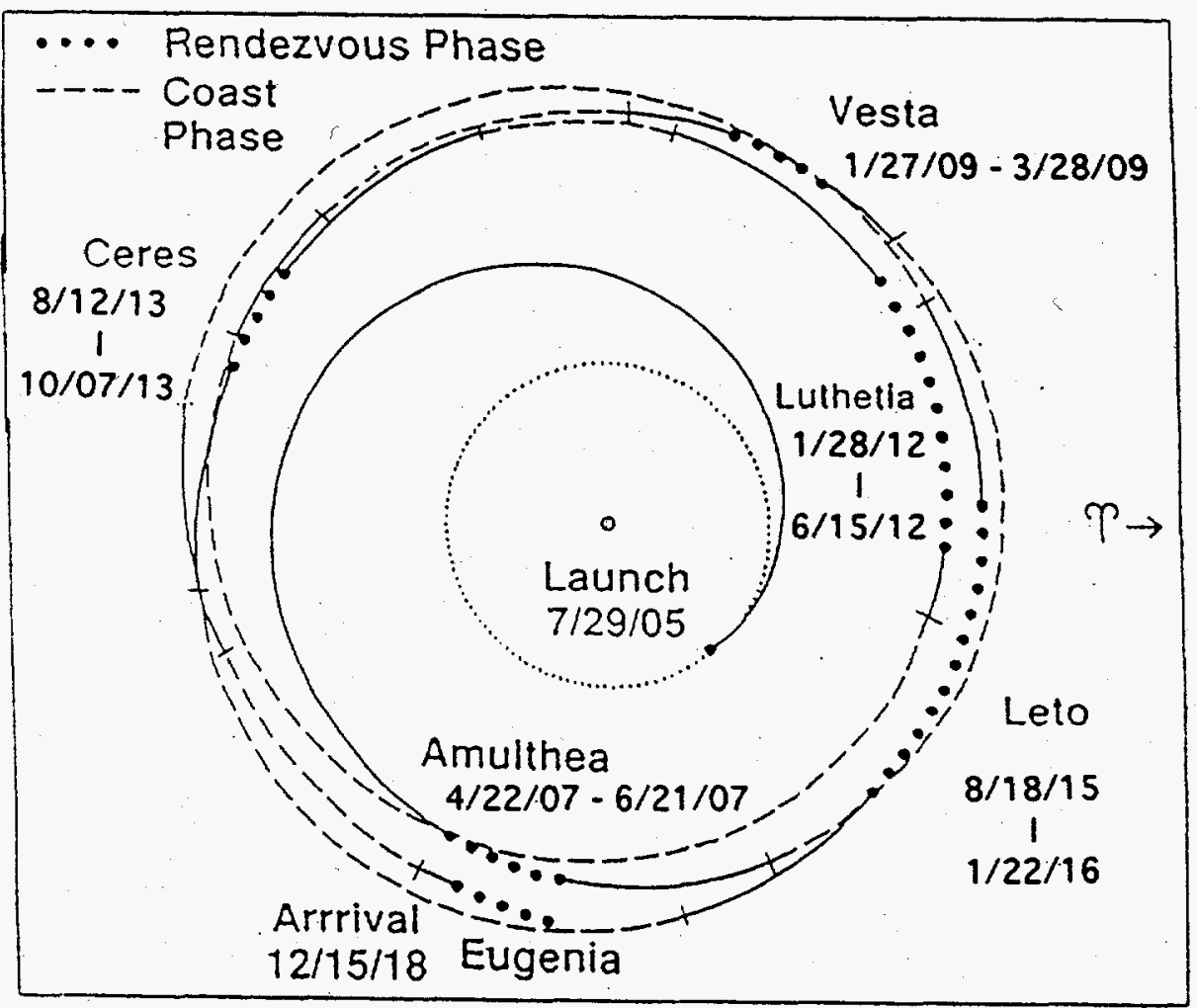

Figure 5-9 2005 Multiple Asteroid Rendezvous Trajectory 


\section{NEP - Ballistic Performance Comparison}

The past studies of Yen and Sauer 32,33 provide an overview of ballistic mission possibilities. These indicate that, using a Titan IV(SRMU)/Centaur, a MMK II spacecraft may be able to rendezvous with two asteroids but one of which will be basically a target of opportunity and unlikely to be a very interesting object. This scenario will involve double swingbys of Mars and take about eight years to attain. The capability of a chemical propulsion system also limits the encounters only to asteroids residing in the innermost belt. This situation is slightly improved if a HLV/Centaur is made available, may be in terms of choice of targets, but the maximum number of targets still will be limited to two for the MMK II class of spacecraft.

The NEP MMBAR example contains six prime (size and diverse class, see performance summary) targets and takes about 10 years with a HLV/Centaur launch and slightly longer with a Titan IV(SRMU)/Centaur launch.

The superiority of NEP over the ballistic option resides in the large number of asteroids accessed, the time it takes to do it and its ability to access choice targets. Other attractive features of an NEP operated MMBAR mission are: 1) the first science is returned relatively quickly in about two years; 2) it will be repeated at about the same rate; 3 ) the mission is risk tolerant in that even with a partially failed NEP system the mission may be redesigned to go on, instead of being a total loss.

\subsubsection{Comet Nucleus Sample Return}

\section{Ballistic Mission Concept.}

An example CNSR concept is that of ROSETTA ${ }^{36-37}$ (was an ESANASA cooperative mission). The mission scenario at a comet, spanning a minimal of 90 days, consists of: 1) high resolution imaging with the objective of selecting a landing site; 2) landing of MMK II spacecraft (1350 kg) equipped with sampling devices and an in-situ laboratory $(\sim 430 \mathrm{~kg})$, and Earth retum aero-capsule $(-300 \mathrm{~kg})$; and 3$)$ collection of $\sim 20 \mathrm{~kg}$ of samples. After the sample collection, the MMK II spacecraft with Earth return aero-capsule will be ejected from the comet to return to Earth. The return speed at Earth is large $\left(v_{\infty}>10\right.$ $\mathrm{km} / \mathrm{s})$. Only the aero-capsule is released for direct Earth entry on return. Preservation of sample, control of g-load and entry corridor control during the high speed atmosphere entry are some of the challenges dictating the design of the re-entry capsule.

\section{NEP Mission Concept}

One NEP version of the mission may be as follows: 1) NEP, main spacecraft, lander-sampler and earth return capsule (aerocapsule) will be sent to rendezvous with a desirable (relatively active/new) comet; 2) the main spacecraft is used for round trip guidance, control, command and communications; it also performs the high resolution imaging needed for landing site selection; 3 ) the main spacecraft remains with the NEP; 4) the lander vehicle with aerocapsule lands on the comet and collects samples, the lander $(500 \mathrm{~kg})$ will be left on the comet; 4) the acquired samples and earth-return capsule $(-450 \mathrm{~kg})$ are designed 10 ascend from the comet and dock with the main spacecraft to travel back to Earth using the NEP ; 5) upon arrival at Earth the sample capsule may be released for direct atmospheric entry or captured via on-orbit recovery.

This scenario differs from the ballistic mode in that the MMK II spacecraft is used for the round trip. The Earth return mass of $1800 \mathrm{~kg}$ (instead of only $300 \mathrm{~kg}$ for the ballistic case) will approach Earth at zero relative velocity $\left(v_{\infty}=0 \mathrm{~km} / \mathrm{s}\right)$ and will have the option to spiral into Earth orbit.

\section{NEP Performance Summary}

Table 5-6 summarizes the performance of a CNSR mission attainable with a NEP for five relatively active short period comets. Only the missions using (HLV/Centaur + NEP) are shown. The 
analysis so far indicates that the performance using Titan IV(SRMU)/Centaur cannot deliver the payload assumed in a reasonable time (e.g. $<10$ years). Due to the eccentric nature of the comet orbit, the performance is sensitive to the launch and return dates which must be optimized and are shown in Table 56.

Performance Using HLV/Centaur + NEP: An acceptable performance will require a flight time of 6.7 to 7.6 years for the example targets used. This flight time is associated with a class of trajectory (called indirect, see Figure 5-10) requiring about 1.5 revolutions about the sun in going to the comet. No significant performance margin exists. Additional margin if needed will require another class of indirect trajectory involving $1.5 \mathrm{rev}$ about the sun for both the outbound and the inbound legs and would take nearly two years longer. The NEP system requirement for this mission is different from the others included in the study. The ISP is relatively low ( $<5000$ seconds), and the thrust time is short; $\sim 4$ years compared to other missions. The reason for the above behavior can be attributed to the eccentric nature of the comet orbits.

\section{NEP Comet Nucleus Sample Return Mission Trajectory}

An example heliocentric transfer trajectory (ecliptic projection) is shown in Figure 5-10.

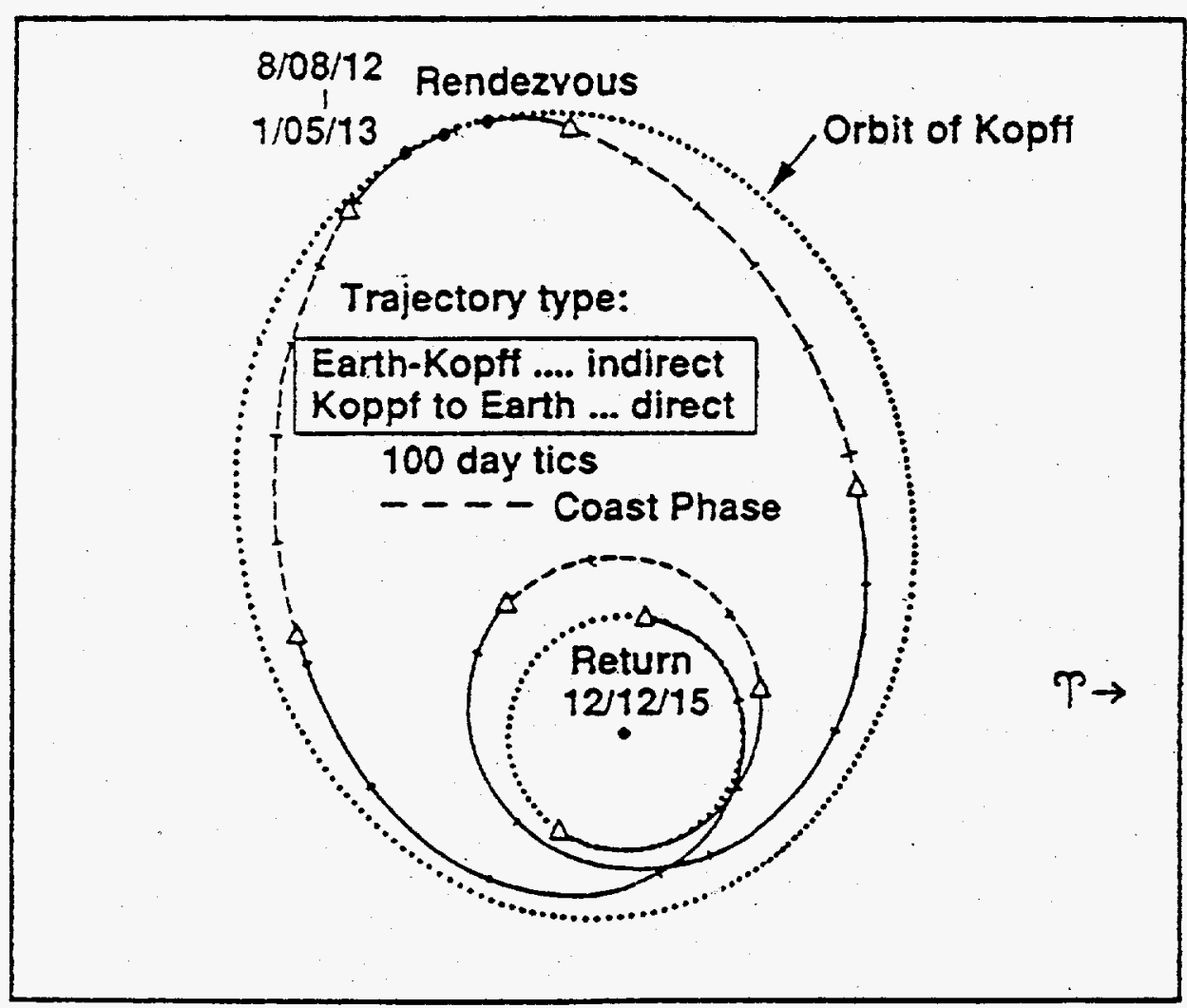

Figure 5-10 2008 Kopff Nucleus Sample Return Trajectory

\section{NEP - Ballistic Performance Comparison}

Ballistic CNSR performance possibilities have been studied quite extensively by Saver ${ }^{38}$. A lack of opportunity to access desirable targets appears to be the main drawback. The capability of the presently available Titan IV(SRMU)/Centaur is such that a mission is possible only with multiple gravity assists of Venus and Earth resulting in flight times of 8.5 to 10.5 years. If a HLV/Centaur class launch vehicle 
becomes available, CNSR missions to a few relatively easily accessible comets are possible using a $\Delta \mathrm{V}$ EGA trajectory with a typical round trip time of 7 to 8 years.

One anticipated benefit of NEP is to gain frequent opportunities to access a greater number of comets of interest (active, fresh comets). Additionally, the preservation of the sample during the return trip is made easier with the ample power of NEP. If direct earth entry can be avoided with an in orbit recovery, NEP will be helpful in attaining the key science goal of "pristine sample preservation" by not subjecting the sample to a high shock environment of a direct earth entry.

\subsubsection{1-AU Solar Polar Orbiter}

\section{Ballistic Mission Concept}

This is one of the most difficult missions to achieve using chemical propulsion. There are two distinct orbital requirements, inclination of $90^{\circ}$ and a 1-AU circular orbit. The use of Jupiter gravity assist can easily deliver the first requirement, but the maneuver required to circularize the elliptic orbit which connects the Earth and Jupiter orbits is a very large one, approximately $18 \mathrm{~km} / \mathrm{s}$. Past investigations concluded that by using repeated Earth gravity assists and flight times of over eight years, a 1-AU circular orbit with an inclination of $60^{\circ}$ is the best achievable orbital configuration, irrespective of the launch vehicle used, either Titan IV or HLV.

\section{NEP Mission Concept}

There are several conceptually different classes of NEP trajectories that can be used to attain a 1 AU solar polar orbit. However, there have been substantial difficulties in achieving convergence to arrive at satisfactory trajectories. So far only one class of flight path has been found which can deliver more than the required $\sim 2500 \mathrm{~kg}$ payload using a (HLV/Centaur + NEP). The NEP is used to thrust the spacecraft about the sun until it reaches a large solar distance $(-5 \mathrm{AU})$ where the bulk of the inclination change burns are made. Starting at that point the spacecraft starts to move inward toward the sun, the elliptic orbital shape is changed by continuous thrusting until it reaches 1-AU circular (see Figure 5-11).

\section{NEP Performance Summary}

Table 5-7 summarizes the NEP mission parameters as a function of flight time and the associated NEP system parameters.

Performance Using Titan IV(SRMU)/Centaur + NEP: This option was not considered in view of the difficulty of the mission. It is expected not to be a viable option for the science payload considered.

Performance Using HLV/Centaur + NEP: The mission may be achieved in 7.75 years using a $96 \mathrm{~kW}$ SRPS and thrusters with a specific impulse of 7200 seconds. The performance improvement with an increase in flighi time is not significant and the mission will not be able to tolerate a NEP system mass growth of more than about $10 \%$. The conclusion is that the mission is difficult and the applicability of the near term NEP for this mission depends on the ability to control the system mass. Note that the NEP requirements are comparable to the outer planet missions but may require longer SP-100 life. . Although no concrete solar monitoring duration has been specified it is anticipated that, at a minimum, a period of one solar cycle $\sim 11$ years will be desirable which in turn imposes a long lifetime $(\sim 20$ years $)$ on the power plant design.

\section{1-AU Solar Polar Orbiter Mission Trajectory}

The heliocentric trajectory for the 7.75 year mission is shown in Figure 5-11. Two plots, an equatorial plane projection of the orbit and the other showing the out-of-plane orbit components, are given to illustrate the changes of orbit shape and orientation with time. Until $\sim 1200$ days into the mission the 
spacecraft travels near the equatorial plane but after 1200 days orbit inclination changes are made and the spacecraft begins to get out of the plane.
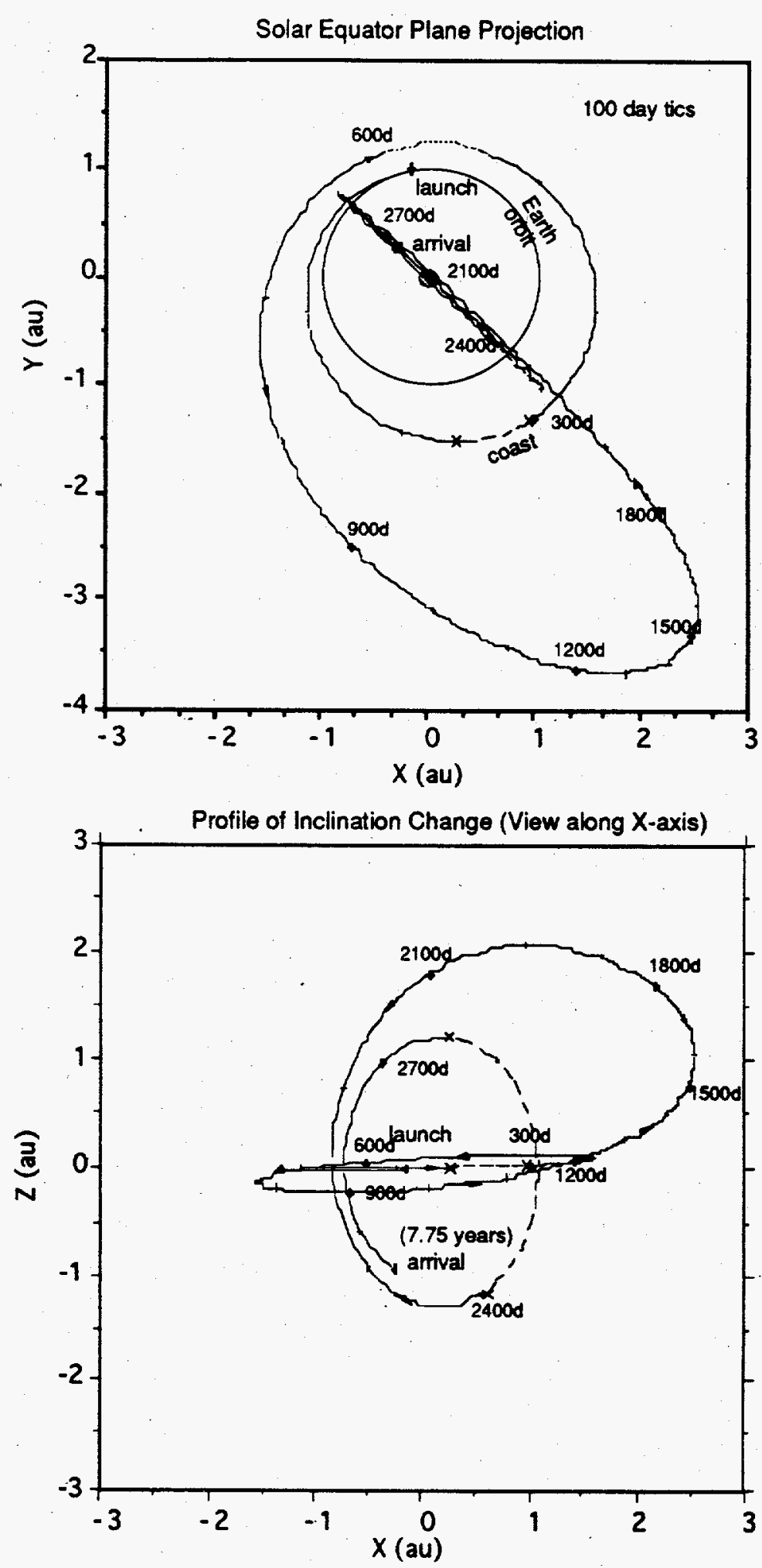

Figure 5-11 1-AU Solar Polar Orbiter Mission Heliocentric Trajectory 


\section{NEP-Ballistic Performance Comparison}

The performance capability of (HLV/Centaur + NEP) versus flight time, given in Table 5-7 is plotted in Figure 5-12. The figure does not include ballistic data points because no viable ballistic option has been found to date.

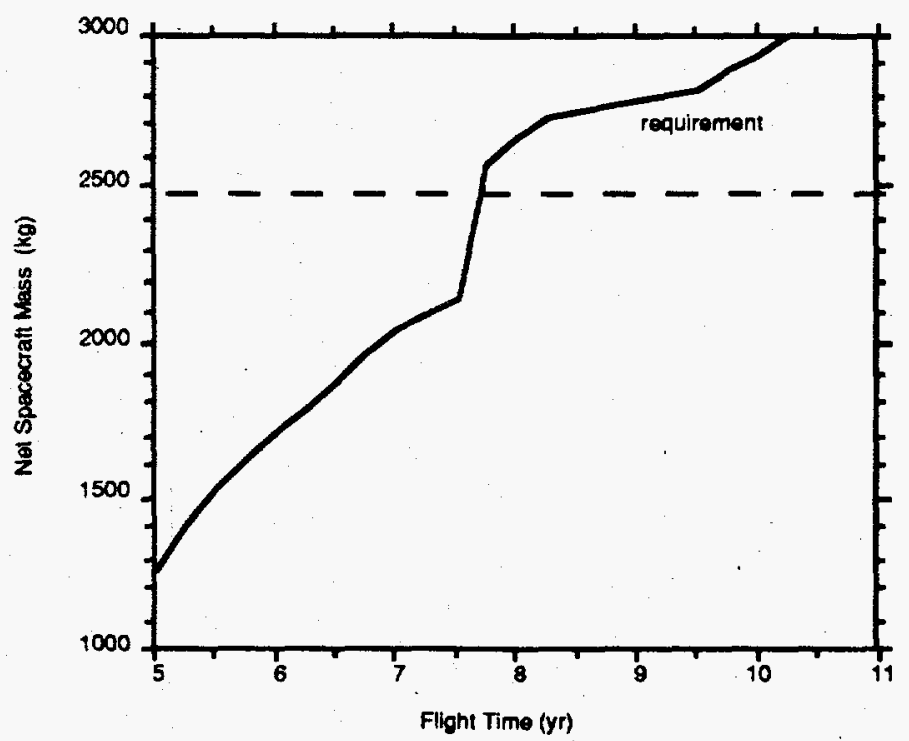

Figure 5-12 1-AU Solar Polar Performance vs. Flight Time

\subsubsection{Pioneer Interstellar Probe}

\section{Ballistic Mission Concept}

The challenge of the mission is to reach $200 \mathrm{AU}$ in less than 25 years. The most effective location to apply a $\Delta V$ to boost the spacecraft solar system escape speed is near the sun (as close as a few solar radii). The current mission scenario calls for first, a swingby of Jupiter to bring about the close flyby of the sun at 4 solar radii (Rs), followed by a $\Delta V$ of $4-6 \mathrm{~km} / \mathrm{s}$ at a solar range of $4-7 \mathrm{Rs}$. The flight system design challenges facing such a concept are thermal control, spacecraft life and flight system mass control.

\section{NEP Mission Scenario}

A NEP interstellar probe would spiral out from a nuclear-safe $1000 \mathrm{~km}$ parking orbit and continue to thrust until a desired solar system escape velocity ( 12.5 AU/year) is attained. A direct earth escape will result in insufficient performance Conceptually, a fuel efficient NEP can continuously thrust until a large solar system escape velocity is attained but the reality of the assumed NEP system mass characteristic is such that an attractive acceleration level cannot be attained to do the mission in a shorter time.

\section{NEP Performance Summary}

Table 5-8 summarizes NEP mission performance as a function of flight time and provides the associated NEP system parameters. The power levels from $200 \mathrm{~kW}$ to $1 \mathrm{MW}$ are shown. Two important parameters in the table are the MPL (net spacecraft mass) and the Mo (initial mass at $1000 \mathrm{~km}$ Earth parking orbit). The MPL represents a mission requirement and the M0 represents a launch vehicle requirement. 


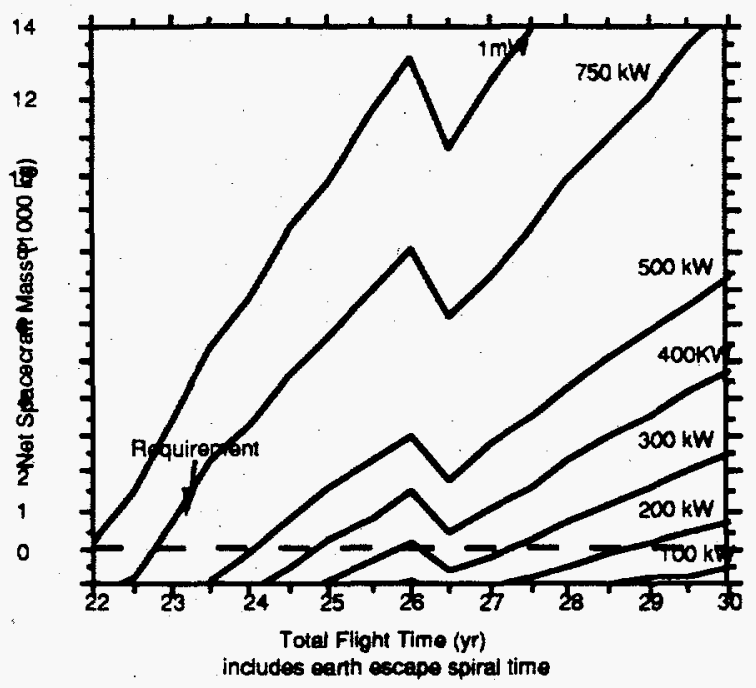

Figure 5-13.1 Pioneer Interstellar Probe Performance

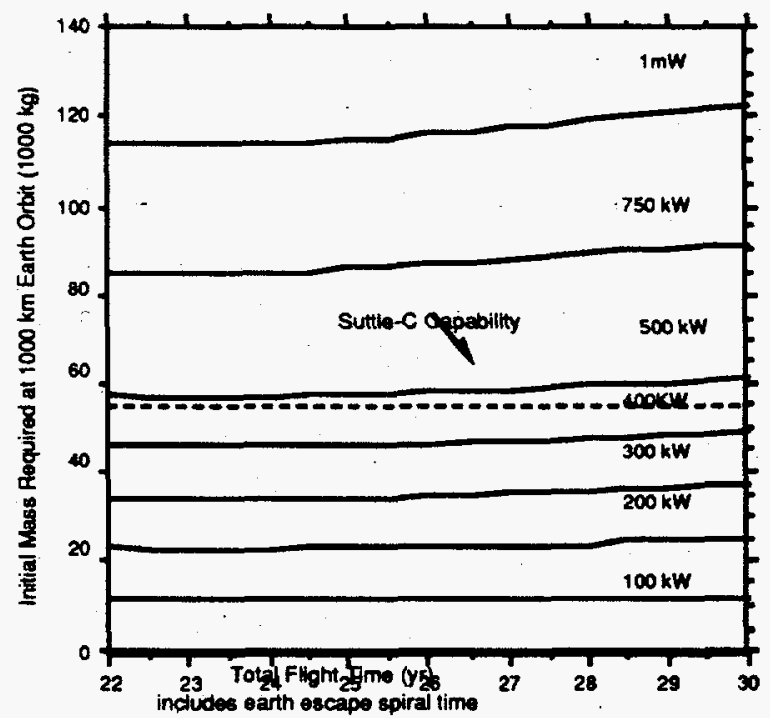

Figure 5-13.2 Pioneer Interstellar Probe Initial Mass Requirements

Current net spacecraft mass requirement is approximately $1000 \mathrm{kgs}$ and the assumed HLV delivery capability to a $1000 \mathrm{~km}$ parking orbit is $-55,000 \mathrm{kgs}$. Figures 5-13.1 and 5-13.2 show the plots of MPL and Mo vs. flight times. These figures lead one to conclude that the Pioneer Interstellar Probe with a flight time of 25 years (minimum requirement) will require a nominal power of $400 \mathrm{~kW}$ and an initial mass of $45000 \mathrm{~kg}$ at $1000 \mathrm{~km}$ Earth orbit. Figure 5-13.1 indicates that given a $1 \mathrm{MW}$ NEP and an arbitrarily large lift capability the shortest mission time attainable is 22 years. But the message from Figure 5-13.2 is that the assumed $\mathrm{HLV}$ is not able to launch a NEP system greater than $500 \mathrm{~kW}$. It is noteworthy that Mo varies little with the flight time. 


\section{NEP Pioneer Interstellar Probe Mission Trajectory}

The NEP trajectory for this mission is shown in Figure 5-14. Two plots showing the trajectory of the early phase, shortly after spiral Earth escape, and the trajectory up to the thrust cut-off are included. The path after the thrust cut-off is a near straight line extension into interstellar space.
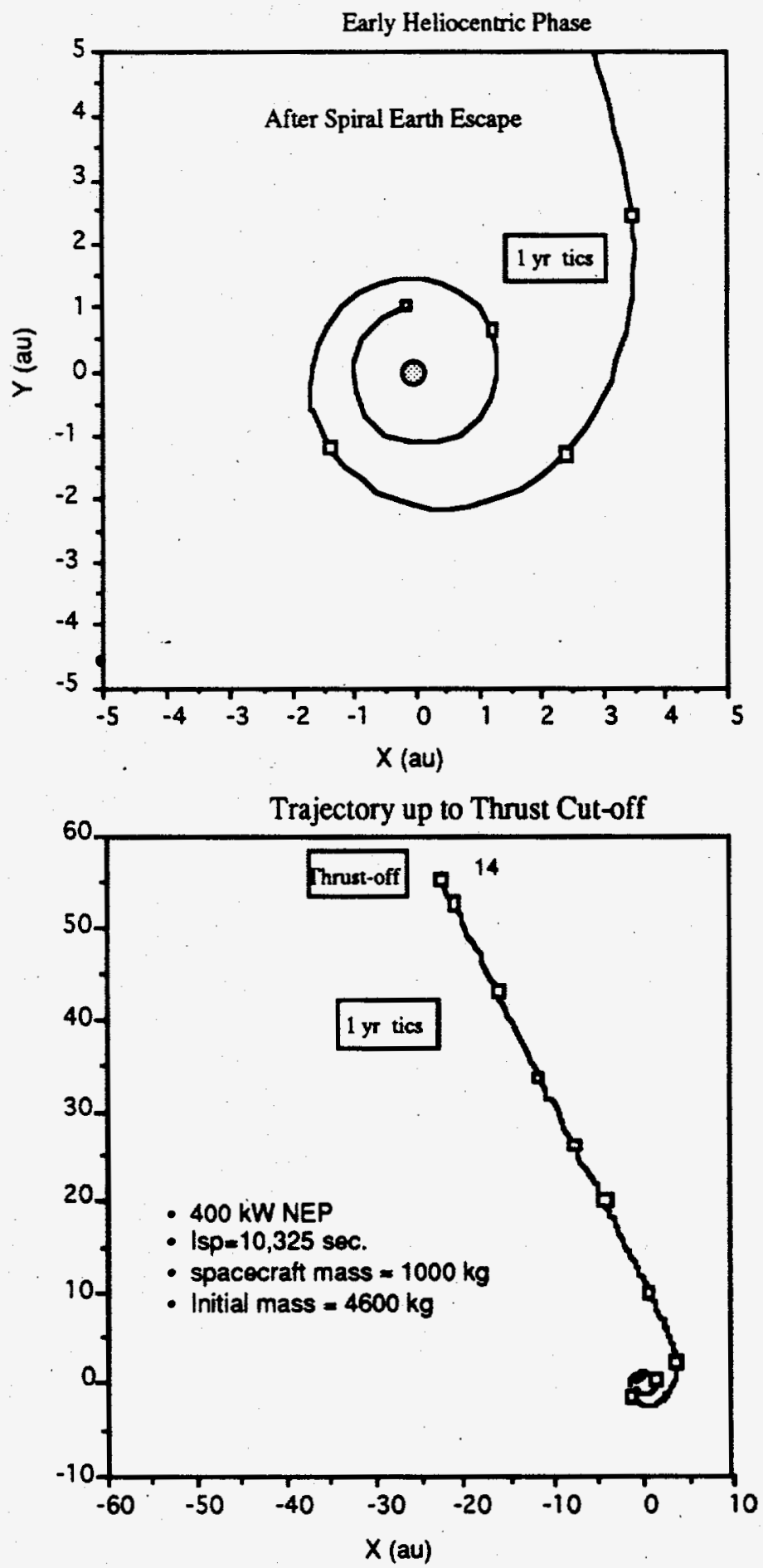

Figure 5-14 NEP Pioneer Interstellar Mission Trajectory 


\section{NEP - Ballistic Performance Comparison}

Given a HLV/Centaur, the chemical option can deliver a $200-400 \mathrm{~kg}$ spacecraft in 25 years to $200 \mathrm{AU}$ using a sun-grazing trajectory. Relative to the ballistic option, the NEP may be able to deliver slightly more mass $(1000 \mathrm{~kg}$ ) with a $400 \mathrm{~kW}$ NEP system and a 14 year full-power thrust and a $>25$ year-life capability. However, the NEP technology of year 2000 represented here appears to only marginally satisfy this mission requirement. The issue is the mission time. Ggiven an advanced propulsion system such as a NEP, the expectation is that a faster mission should be within reach but this expectation cannot be met with a NEP system which has an overall specific mass of $>50 \mathrm{~kg} / \mathrm{kW}$. The reason is that such a system simply is not able to attain the high acceleration level needed (even with a zero payload) to do a fast interstellar trip. A more advanced mega-watt system with much smaller specific mass will be more appropriate for this mission.

\subsubsection{Performance Data (Tables)}

Results of the performance analysis is tabulated in this section. The performance capability and NEP system requirements versus heliocentric flight times are provided. The performance achieved using both launch vehicles, a Titan IV(SRMU)/Centaur and a HLV/Centaur, are shown. Summary notes commenting on the results are appended to the table for easier comprehension. The purpose of this rather extensive data display is to provide sufficient information to understand the sensitivity of mission feasibility with respect to various NEP system component uncertainties. Given a NEP system which is massive (e.g. $5500 \mathrm{~kg} @ 100 \mathrm{~kW}$ ) compared to the payload delivered (e.g. $1500 \mathrm{~kg}$ ), a $25 \%$ uncertainty in the NEP system mass assumption (or the level of confidence in system representation), which is not uncommon at an early stage of design, can totally invalidate the mission concept unless a sufficient performance margin is indicated in the performance tables.

The performance summary tables follows one format for all the missions discussed and the nomenclature used for the tables are as follows.

\section{Nomenclature for performance summary tables}

FT Heliocentric transfer time

FT1 Flight time including planetary spiral phase

VHL Earth escape $V_{\infty}$

PO Input power to PPU

ISP Specific impulse

$P_{R} \quad$ Thruster power rating

$\mathrm{P}_{\mathrm{O}} \quad$ Operating power level of thruster

$\mathrm{T}_{\mathrm{L}} \quad$ Thruster life at operating power

$T_{\mathbf{p}} \quad$ Total propulsion time

$\mathrm{N}_{\mathrm{O}} \quad$ No.of operating thrusters per segmented engine
$N_{I} \quad$ Total no. of thrusters (ion sources) installed

$\mathrm{N}_{\text {PPU }} \quad$ No. of power processor units installed

$M_{0} \quad$ Launch mass minus adapter

MP Propellant mass

MPP SRPS mass

$M_{\text {TH }} \quad$ Thrust subsystem dry mass (including tankage)

$M_{\text {NEP }} \quad$ Total NEP system mass $(d r y)=\left(M_{P P}+M_{T H}\right)$

$M_{P L} \quad$ Maximum payload delivered to final orbit

$V_{A C}$ Total characteristic velocity of the mission 
Table 5-1.1 Pluto Orbiter/P(optional) Performance Summary Requirement: $\mathrm{MPL} \geq 1400 \mathrm{~kg}$

PLO/P with (Titan IV(SRMU)/Centaur +NEP)

\begin{tabular}{|c|c|c|c|c|c|c|c|c|c|c|c|c|c|c|c|c|c|c|}
\hline $\begin{array}{c}\mathrm{PT} \\
(\mathrm{yr})\end{array}$ & $\begin{array}{l}\text { FII } \\
(y r)\end{array}$ & $\begin{array}{l}\text { VHL } \\
(\mathrm{km} / \mathrm{s}\end{array}$ & 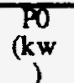 & $\begin{array}{c}\mathbf{S P} \\
(\mathrm{sec})\end{array}$ & $\begin{array}{c}P_{R} \\
(\mathbf{k w})\end{array}$ & $\underset{(k w)}{P_{0}}$ & $\begin{array}{l}\mathrm{I}_{\mathrm{L}} \\
(\mathrm{yr})\end{array}$ & $\begin{array}{c}T_{P} \\
(y r)\end{array}$ & $\mathbf{N}_{0}$ & $\mathbf{N}_{\mathrm{I}}$ & $\mathrm{N}_{\mathrm{PPU}}$ & $\begin{array}{l}M_{0} \\
(\mathbf{k g})\end{array}$ & $\underset{(\mathrm{kg}}{\mathrm{M}_{\mathrm{g}}}$ & $\underset{\left(k_{B}\right)}{M_{p}}$ & $\underset{(\mathbf{g})}{\mathrm{M}_{\mathrm{TH}}}$ & $\underset{(\mathrm{kg})}{\mathrm{M}_{\mathrm{N}}}$ & $\underset{\left(k_{B}\right)}{M_{f}}$ & $\underset{(\mathrm{km} / 2)}{\mathrm{V}_{\mathrm{AC}}}$ \\
\hline 13.5 & 13.5 & 2.4 & 58 & 8095 & 13 & 12 & 1.32 & 7.8 & 5 & 40 & 2 & 8315 & 3134 & 2844 & 1162 & 4006 & 1175 & 37.6 \\
\hline 14.0 & 14.0 & 2.4 & 57 & 8238 & 14 & 11 & 1.37 & 7.9 & 5 & 40 & 2 & 8303 & 3009 & 2829 & 1143 & 3972 & 1322 & 36.4 \\
\hline 14.5 & 14.5 & 2.4 & 56 & 8358 & 14 & 11 & 1.41 & 8.0 & 5 & 40 & 2 & 8301 & 2905 & 2815 & 1127 & 3942 & 1454 & 35.3 \\
\hline 15.0 & 15.0 & 2.4 & 56 & 8461 & 14 & 14 & 1.15 & 8.0 & 4 & 36 & 2 & 8314 & 2822 & 2804 & 1079 & 3883 & 1609 & 34.4 \\
\hline 15.5 & 15.5 & 2.3 & 55 & 8556 & 14 & 14 & 1.18 & 8.1 & 4 & 36 & 2 & 8351 & 2763 & 2800 & 1070 & 3870 & 1718 & 33.7 \\
\hline 16.0 & 16.0 & 1.0 & 58 & 9390 & 16 & 15 & 1.22 & 10.3 & 4 & 44 & 2 & 8967 & 3075 & 2989 & 1192 & 4181 & 1711 & 38.7 \\
\hline 16.5 & 16.5 & 1.0 & 57 & 9617 & 16 & 14 & 1.28 & 10.6 & 4 & 44 & 2 & 8952 & 2964 & 2980 & 1172 & 4152 & 1836 & 37.9 \\
\hline 17.0 & 17.0 & 1.1 & 56 & 9812 & 16 & 14 & 1.33 & 10.9 & 4 & 44 & 2 & 8931 & 2856 & 2968 & 1152 & 4120 & 1955 & 37.1 \\
\hline 17.5 & 17.5 & 1.2 & 55 & 9979 & 17 & 14 & 1.38 & 11.1 & 4 & 44 & 2 & 8909 & 2755 & 2953 & 1134 & 4087 & 2067 & 36.2 \\
\hline 18.0 & 18.0 & 1.2 & 54 & 10121 & 17 & 13 & 1.43 & 11.2 & 4 & 40 & 2 & 8887. & 2662 & 2937 & 1083 & 4020 & 2205 & 35.3 \\
\hline $\begin{array}{l}\cdot \mathrm{M} \\
\cdot \mathrm{F} \\
\cdot \mathrm{N}\end{array}$ & 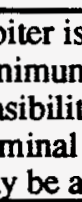 & is 1 & 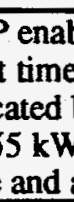 & (nen: & 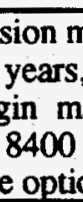 & 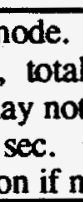 & 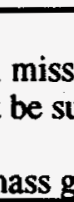 & & & & & & & & & & , & \\
\hline
\end{tabular}

Table 5-1.2 Pluto Orbiter/P(optional lander) Performance Summary (continued) Requirement: $\quad$ MPL $\geq 1410 \mathrm{~kg}$

PLO/P with (HLV/Centaur +NEP)

\begin{tabular}{|c|c|c|c|c|c|c|c|c|c|c|c|c|c|c|c|c|c|c|}
\hline $\begin{array}{c}F T \\
(y r)\end{array}$ & $\begin{array}{l}\text { FII } \\
\text { (yr) }\end{array}$ & $\begin{array}{l}\text { VHL } \\
(\mathrm{km} / \mathrm{s}\end{array}$ & $\begin{array}{c}105 \\
(\mathbf{k w})\end{array}$ & $\begin{array}{c}\mathrm{SPP} \\
(\mathrm{sec})\end{array}$ & $\underset{(\mathbf{k} w)}{\mathbf{P}_{\mathbf{R}}}$ & $\left(\begin{array}{l}P_{0} \\
\mathbf{w}\end{array}\right)$ & $\begin{array}{l}\mathrm{r}_{\mathrm{L}} \\
(\mathrm{yr})\end{array}$ & $\begin{array}{c}T_{P} \\
(y r)\end{array}$ & $\mathbf{N}_{0}$ & $\mathbf{N}_{\mathbf{I}}$ & $\mathrm{N}_{\mathrm{FPU}}$ & $\begin{array}{l}M_{0} \\
(x)\end{array}$ & $\underset{\left(\mathrm{KB}_{\mathrm{B}}\right.}{\mathrm{M}_{\mathrm{P}}}$ & $\underset{(\mathrm{kg})}{\mathrm{M}_{\mathrm{P}}}$ & $\underset{(\mathbf{g})}{\mathbf{M}_{\mathrm{TH}}}$ & $\underset{\mathbf{R}_{\mathbf{g}}}{\mathrm{M}_{\mathrm{N}}}$ & $\underset{\left(k_{g}\right)}{M_{F}}$ & $\begin{array}{l}V_{A C} \\
(k+n / n)\end{array}$ \\
\hline 11.0 & 11.0 & 1.8 & 103 & 6884 & 11 & 11 & 1.14 & 6.8 & 9 & 72 & 2 & 13659 & 6671 & 3682 & 2132 & 5813 & 1175 & 45.2 \\
\hline 11.5 & 11.5 & 1.8 & 103 & 7157 & 12 & 11 & 1.19 & 7.0 & 9 & 72 & 2 & 13706 & 6337 & 3691 & 2097 & 5788 & 1581 & 43.6 \\
\hline 12.0 & 12.0 & 1.8 & 102 & 7396 & 12 & 11 & 1.24 & 7.2 & 9 & 72 & 2 & 13734 & 6034 & 3691 & 2062 & 5753 & 1947 & 42.0 \\
\hline 12.5 & 12.5 & 1.7 & 101 & 7602 & 13 & 13 & 1.15 & 7.3 & 8 & 64 & 2 & 13761 & 5770 & 3684 & 1961 & 5645 & 2346 & 40.5 \\
\hline 13.0 & 13.0 & 1.7 & 100 & 7777 & 13 & 12 & 1.18 & 7.4 & 8 & 64 & 2 & 13798 & 5547 & 3675 & 1933 & 5608 & 2643 & 39.2 \\
\hline 13.5 & 13.5 & 1.7 & 99 & 7929 & 13 & 12 & 1.22 & 7.5 & 8 & 64 & 2 & 13857 & 5368 & 3668 & 1910 & 5578 & 2911 & 38.1 \\
\hline 14.0 & 14.0 & 1.6 & 99 & 8070 & 13 & 12 & 1.24 & 7.7 & 8 & 64 & 2 & 13955 & 5238 & 3669 & 1894 & 5563 & 3154 & 37.2 \\
\hline 14.5 & 14.5 & 1.4 & 99 & 8245 & 14 & 12 & 1.27 & 7.9 & 8 & 64 & 2 & 14163 & 5197 & 3697 & 1894 & 5591 & 3375 & 37.0 \\
\hline 15.0 & 15.0 & 0.8 & 100 & 8736 & 15 & 14 & 1.16 & 9.1 & 7 & 70 & 2 & 14610 & 5371 & 3826 & 1971 & 5797 & 3442 & 39.3 \\
\hline 15.5 & 15.5 & 0.7 & 98 & 9077 & 15 & 14 & 1.23 & 9.7 & 7 & 70 & 2 & 14647 & 5240 & 3839 & 1941 & 5780 & 3627 & 39.4 \\
\hline 16.0 & 16.0 & 0.7 & 96 & 9335 & 16 & 14 & 1.29 & 10.2 & 7 & 70 & 2 & 14638 & 5067 & 3831 & 1909 & 5740 & 3831 & 38.9 \\
\hline $\begin{array}{l}\cdot I \\
\cdot I \\
\cdot I\end{array}$ & & - & 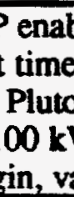 & 1 & 72 & & & & & & & & & & & & & \\
\hline
\end{tabular}


Table 5-2.1 Neptune Orbiter/P Performance Summary Requirement: $\quad \mathrm{MPL}_{\mathrm{PL}} \geq 1400 \mathrm{~kg}$

NEO/P with (Titan IV(SRMU)/Centaur + NEP)

\begin{tabular}{|c|c|c|c|c|c|c|c|c|c|c|c|c|c|c|c|c|c|c|}
\hline $\begin{array}{l}\mathrm{FT} \\
(\mathrm{yr})\end{array}$ & $\begin{array}{l}\text { FII } \\
(y r)\end{array}$ & VHL & $\underset{(k w)}{10}$ & $\underset{(\mathrm{sec})}{\mathrm{SPP}}$ & $\begin{array}{c}P_{\mathrm{R}} \\
(\mathrm{kW})\end{array}$ & $P_{(\mathrm{k} w)}$ & $\begin{array}{l}\mathrm{T}_{\mathrm{L}} \\
(\mathrm{yt})\end{array}$ & $\underset{(y r)}{T_{P}}$ & $N_{0}$ & $N_{1}$ & $N_{\text {pqu }}$ & $\begin{array}{l}\mathrm{M}_{0} \\
(\mathrm{~kg})\end{array}$ & $\mathrm{Mg}_{\mathrm{g}}$ & $\mathrm{M}_{\mathrm{fqg}}$ & $\underset{\left(\mathbf{k}_{\mathrm{g}}\right)}{\mathrm{M}_{\mathbf{H}}}$ & 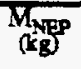 & $\mathrm{M}_{(\mathrm{g})}$ & $V_{(\mathrm{km} / \mathrm{s})}$ \\
\hline 16.0 & 16.9 & 1.1 & 58 & 10000 & 17 & 14 & 1.31 & 11.6 & 4 & 48 & 2 & 8923 & 3035 & 3054 & 1219 & 4274 & 1238 & 41.0 \\
\hline 16.5 & 17.5 & 1.2 & 56 & 10000 & 17 & 14 & 1.37 & 11.8 & 4 & 44 & 2 & 8901 & 2963 & 3010 & 1159 & 4170 & 1392 & 40.0 \\
\hline 17.0 & 18.0 & 1.3 & 54 & 10000 & 17 & 13 & 1.42 & 11.9 & 4 & 44 & 2 & 8878 & 2891 & 2971 & 1137 & 4108 & 1503 & 38.9 \\
\hline 17.5 & 18.6 & 1.3 & 52 & 10000 & 17 & 13 & 1.46 & 12.0 & 4 & 44 & 2 & 8856 & 2823 & 2936 & 1116 & 4052 & 1605 & 37.9 \\
\hline 18.0 & 19.1 & 1.4 & 50 & 10000 & 17 & 13 & 1.51 & 12.1 & 4 & 44 & 2 & 8837 & 2760 & 2905 & 1099 & 4003 & 1698 & 37.0 \\
\hline
\end{tabular}

Table 5-2.2 Neptune Orbiter/P Performance Summary (continued) Requirement: $\quad$ MPL $\geq 1400 \mathrm{~kg}$

NEO/P with (HLV/Centaur + NEP)

\begin{tabular}{|c|c|c|c|c|c|c|c|c|c|c|c|c|c|c|c|c|c|c|}
\hline $\begin{array}{l}F r \\
(y r)\end{array}$ & $\begin{array}{l}\text { FIT } \\
(\mathrm{yr})\end{array}$ & $\begin{array}{l}\text { VHL } \\
(\mathrm{km} / \mathrm{s}\end{array}$ & $\underset{(\mathbf{k} w)}{10}$ & $\underset{(\mathrm{sec})}{\mathrm{SP}}$ & $\underset{(\mathbf{k} w)}{P_{\mathbf{R}}}$ & $\underset{(\mathrm{kw})}{\mathrm{P}_{0}}$ & $\begin{array}{l}T_{L} \\
(y r)\end{array}$ & $\begin{array}{l}T_{p} \\
(y r)\end{array}$ & $N_{0}$ & $N_{1}$ & $N_{\text {PFU }}$ & $\begin{array}{l}\mathrm{M}_{0} \\
(\mathbf{k g})\end{array}$ & $\mathrm{M}_{\mathbf{p}}$ & $\mathrm{M}_{\mathrm{kg}}$ & 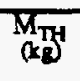 & $\begin{array}{l}\mathrm{M}_{\mathrm{NEP}} \\
(\mathbf{g})\end{array}$ & $\mathrm{M}_{\mathrm{kg}}$ & $\begin{array}{l}\mathrm{V}_{\mathrm{AC}} \\
\mathrm{imm} / \mathrm{km}\end{array}$ \\
\hline 11.5 & 12.0 & 1.9 & 102 & 7565 & 13 & 11 & 1.27 & 7.7 & 9 & 72 & 2 & 13548 & 6185 & 3741 & 2078 & 5818 & 1169 & \\
\hline 12.0 & 12.5 & 1.9 & 101 & 7826 & 13 & 13 & 1.17 & 7.9 & 8 & 72 & 2 & 13580 & 5901 & 3745 & 2046 & 5790 & 1513 & \\
\hline 12.5 & 13.1 & 1.9 & 101 & 8051 & 13 & 13 & 1.22 & 8.1 & 8 & 72 & 2 & 13623 & 5664 & 3746 & 2018 & 5763 & 1820 & \\
\hline 13.0 & 13.6 & 1.8 & 100 & 8248 & 14 & 12 & 1.25 & 8.3 & 8 & 72 & 2 & 13693 & 5477 & 3748 & 1996 & 5744 & 2096 & \\
\hline 13.5 & 14.1 & 1.7 & 100 & 8428 & 14 & 12 & 1.29 & 8.4 & 8 & 72 & 2 & 13813 & 5349 & 3760 & 1982 & 5742 & 2346 & \\
\hline 14.0 & 14.7 & 1.5 & 101 & 8637 & 14 & 14 & 1.14 & 8.8 & 7 & 70 & 2 & 14073 & 5331 & 3806 & 1970 & 5776 & 2590 & \\
\hline 14.5 & 15.2 & 0.8 & 102 & 9136 & 15 & 15 & 1.19 & 10.0 & 7 & 77 & 2 & 14592 & 5517 & 3949 & 2059 & 6007 & 2692 & \\
\hline 15.0 & 15.8 & 0.8 & 100 & 9476 & 16 & 14 & 1.26 & 10.7 & 7 & 77 & 2 & 14615 & 5358 & 3959 & 2026 & 5985 & 2896 & \\
\hline 15.5 & 16.4 & 0.8 & 98 & 9755 & 16 & 14 & 1.33 & 11.1 & 7 & 77 & 2 & 14599 & 5167 & 3953 & 1992 & 5944 & 3112 & \\
\hline 16.0 & 16.9 & 0.8 & 96 & 9996 & 17 & 16 & 1.19 & 11.5 & 6 & 78 & 2 & 14575 & 4978 & 3940 & 1968 & 5908 & 3313 & \\
\hline
\end{tabular}

- Minimum flight time $=12$ years, total mission time $\sim 14$ years.

- Addition of a Triton lander possible @ FT = 13.5 years, total mission time $\sim 15.5$ years.

- Can tolerate NEP mass uncertainty of $30 \% @ \mathrm{FT}=15.5$ years, but thrust time and mission time is longer.

- Nominal $P O \approx 100 \mathrm{~kW}$, ISP $\approx 8000$ seconds.

- Viable option 
Table 5-3.1 Uranus Orbiter/Probe Performance Summary

Requirement: $M_{P L} \geq 1410 \mathrm{~kg}$ (note: probe released from high altitude orbit-24 $R_{u}$ )

UO/P with (Titan IV/Centaur +NEP)

\begin{tabular}{|c|c|c|c|c|c|c|c|c|c|c|c|c|c|c|c|c|c|c|}
\hline $\begin{array}{l}\text { FT } \\
(y r)\end{array}$ & $\begin{array}{l}\text { FII } \\
(y r)\end{array}$ & VHL & $\begin{array}{c}P 0 \\
(k w)\end{array}$ & $\underset{(\mathrm{sec})}{\mathrm{SPP}}$ & $\underset{(\mathbf{k} w)}{\mathbf{P}_{\mathbf{R}}}$ & $\underset{(\mathbf{k} w)}{P_{\mathrm{o}}}$ & $\begin{array}{c}T_{L} \\
(y r)\end{array}$ & $\begin{array}{c}\mathrm{T}_{\mathrm{p}} \\
(\mathrm{yr})\end{array}$ & $N_{0}$ & $N_{1}$ & $N_{\text {PPU }}$ & $\begin{array}{l}\mathrm{M}_{0} \\
(\mathbf{k g})\end{array}$ & $\begin{array}{l}M_{p} \\
(\mathbf{k g})\end{array}$ & $\mathrm{M}_{(\mathbf{p g}}$ & $\underset{(\mathbf{R g})}{\mathrm{M}_{\mathrm{T}}}$ & $\frac{M_{(\mathbf{N E P}}}{\left(\mathbf{K}_{\mathrm{g}}\right)}$ & $\mathrm{Mpg}_{(\mathrm{gg}}$ & $V_{(\mathrm{km} / \mathrm{A})}$ \\
\hline 14.0 & 16.4 & 1.3 & 52 & 10000 & 17 & 13 & 1.47 & 12.1 & 4 & 44 & 2 & 8846 & 2828 & 2936 & 1116 & 4051 & 886 & 39.6 \\
\hline 14.5 & 17.1 & 1.4 & 50 & 10000 & 17 & 17 & 1.15 & 12.3 & 3 & 42 & 2 & 8811 & 2757 & 2895 & 1074 & 3969 & 1004 & 38.6 \\
\hline 15.0 & 17.7 & 1.5 & 48 & 10000 & 17 & 16 & 1.19 & 12.5 & 3 & 42 & 2 & 8782 & 2694 & 2860 & 1054 & 3914 & 1093 & 37.8 \\
\hline 15.5 & 18.3 & 1.5 & 46 & 10000 & 17 & 15 & 1.23 & 12.6 & 3 & 39 & 2 & 8763 & 2642 & 2832 & 1012 & 3844 & 1196 & 37.0 \\
\hline 16.0 & 18.9 & 1.6 & 45 & 10000 & 17 & 15 & 1.26 & 12.7 & 3 & 39 & 2 & 8757 & 2603 & 2810 & 999 & 3809 & 1264 & 36.4 \\
\hline 16.5 & 19.5 & 1.5 & 44 & 10000 & 17 & 15 & 1.28 & 12.9 & 3 & 39 & 2 & 8774 & 2584 & 2797 & 990 & 3787 & 1322 & 36.0 \\
\hline 17.0 & 20.1 & 0.8 & 42 & 10000 & 17 & 14 & 1.36 & 14.8 & 3. & 42 & 2 & 9021 & 2814 & 2825 & 1017 & 3841 & 1285 & 38.5 \\
\hline 17.5 & 20.8 & 0.8 & 40 & 10000 & 17 & 13 & 1.41 & 15.2 & 3 & 42 & 3 & 9005 & 2774 & 2799 & 1165 & 3964 & 1186 & 37.9 \\
\hline 18.0 & 21.4 & 0.9 & 39 & 10000 & 17 & 13 & 1.46 & 15.5 & 3 & 42 & 3 & 8985 & 2728 & 2774 & 1144 & 3919 & 1257 & 37.3 \\
\hline
\end{tabular}

Table 5-3.2 Uranus Orbiter/Probe Performance Summary (continued)

Requirement: $M_{P L} \geq 1410 \mathrm{~kg}$ (note: probe released from high altitude orbit-24 $R_{u}$ )

UO/P with (HLV/Centaur +NEP)

\begin{tabular}{|c|c|c|c|c|c|c|c|c|c|c|c|c|c|c|c|c|c|c|}
\hline $\begin{array}{c}F T \\
(y r)\end{array}$ & $\begin{array}{l}\text { Frit } \\
(\mathrm{yr})\end{array}$ & $\begin{array}{l}\text { VHLL } \\
(\mathrm{km} / \mathrm{s}\end{array}$ & $\underset{(\mathrm{kw})}{\mathrm{PO}}$ & $\underset{(\mathrm{sec})}{\mathrm{TSP}}$ & $\underset{(\mathbf{k w})}{P_{\mathbf{R}}}$ & $\mathbf{P}_{\mathbf{0}}$ & $\begin{array}{l}T_{L} \\
(y r)\end{array}$ & $\begin{array}{l}T_{p} \\
(y r)\end{array}$ & $\mathbf{N}_{0}$ & $\mathrm{~N}_{1}$ & $N_{\text {PFU }}$ & $\begin{array}{l}\mathrm{M}_{0} \\
(\mathrm{~kg})\end{array}$ & (ng & 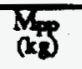 & $\mathrm{M}_{\mathrm{T}} \mathrm{M}_{\mathrm{g}}$ & $M_{\alpha E P}$ & $\mathrm{M}_{\mathrm{Rg}}$ & $\underset{(\mathbf{l n} / 2)}{V_{A C}}$ \\
\hline 10.0 & 11.4 & 1.9 & 99 & 8086 & 13 & 12 & 1.24 & 8.0 & 8 & 72 & 2 & 13511 & 5474 & 3704 & 1986 & 5689 & 1267 & 42.5 \\
\hline 10.5 & 12.1 & 1.9 & 98 & 8365 & 14 & 14 & 1.14 & 8.3 & 7 & 70 & 2 & 13534 & 5231 & 3703 & 1936 & 5639 & 1583 & 41.4 \\
\hline 11.0 & 12.7 & 1.9 & 97 & 8601 & 14 & 14 & 1.18 & 8.6 & 7 & 70 & 2 & 13626 & 5071 & 3713 & 1917 & 5630 & 1844 & 40.5 \\
\hline 11.5 & 13.3 & 1.6 & 98 & 8831 & 15 & 14 & 1.20 & 8.9 & 7 & 70 & 2 & 13885 & 5045 & 3760 & 1921 & 5681 & 2078 & 40.3 \\
\hline 12.0 & 13.9 & 0.8 & 101 & 9356 & 16 & 14 & 1.24 & 10.3 & 7 & $\pi$ & 2 & 14588 & 5328 & 3942 & 2031 & 5973 & 2206 & 42.9 \\
\hline 12.5 & 14.6 & 0.8 & 98 & 9752 & 16 & 14 & 1.32 & 11.0 & 7 & $\pi 7$ & 2 & 14590 & 5134 & 3952 & 1992 & 5944 & 2431 & 42.6 \\
\hline 13.0 & 15.3 & 0.9 & 96 & 10093 & 17 & 16 & 1.20 & 11.6 & 6 & 78 & 2 & 14553 & 4913 & 3945 & 1961 & 5906 & 2653 & 41.9 \\
\hline 13.5 & 16.0 & 0.9 & 94 & 10388 & 17 & 16 & 1.26 & 12.0 & 6 & 72 & 2 & 14510 & 4704 & 3931 . & 1873 & 5805 & 2920 & 41.1 \\
\hline 14.0 & 16.6 & 1.0 & 92 & 10640 & 18 & 15 & 1.32 & 12.3 & 6 & 72 & 2 & 14472 & 4521 & 3915 & 1841 & 5756 & 3114 & 40.2 \\
\hline 14.5 & 17.3 & 1.0 & 90 & 10855 & 18 & 18 & 1.14 & 12.6 & 5 & 70 & 2 & 14451 & 4371 & 3901 & 1797 & 5698 & 3301 & 39.5 \\
\hline 15.0 & 17.9 & 1.0 & 89 & 11050 & 18 & 18 & 1.18 & 12.9 & 5 & 70 & 2 & 14460 & 4258 & 3896 & 1777 & 5673 & 3448 & 38.9 \\
\hline 15.5 & 18.5 & 0.9 & 88 & 11274 & 19 & 18 & 1.21 & 13.4 & 5 & 70 & 2 & 14535 & 400 & 3917 & 1767 & 5684 & 3570 & 38.8 \\
\hline 16.0 & 19.2 & 0.6 & 88 & 11761 & 20 & 18 & 1.27 & 14.7 & 5 & 75 & 2 & 14712 & 4223 & 4012 & 1809 & 5821 & 3587 & 40.1 \\
\hline$\cdot 1$ & . & 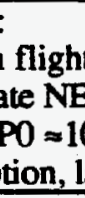 & & & & 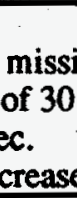 & & & & & & & & & & & & \\
\hline
\end{tabular}


Table 5-4.1 Jupiter Grand Tour Performance Summary

Requirement: MPL $\geq 1434 \mathrm{~kg}$ (Excess performance are for probes or for two JPO spacecraft)

JGT with Titan IV(SRMU)/Centaur +NEP

\begin{tabular}{|c|c|c|c|c|c|c|c|c|c|c|c|c|c|c|c|c|c|c|}
\hline $\begin{array}{c}F T \\
(y r)\end{array}$ & $\begin{array}{l}\text { FII } \\
\text { (yr) }\end{array}$ & $\begin{array}{l}\text { VHL } \\
(\mathrm{km} / \mathrm{s}\end{array}$ & $\underset{(k w)}{P 0}$ & $\begin{array}{c}\text { ISP } \\
(\mathrm{sec})\end{array}$ & $\underset{(\mathbf{k} \mathbf{w})}{\mathbf{P}_{\mathbf{R}}}$ & ${ }_{(\mathrm{k} w)}^{P_{0}}$ & $\begin{array}{l}T_{L} \\
(y r)\end{array}$ & $\begin{array}{c}T_{p} \\
(y r)\end{array}$ & $\mathrm{N}_{0}$ & $N_{1}$ & $\mathrm{X}_{\mathrm{PPU}}$ & $\begin{array}{l}\mathrm{M}_{0} \\
(\mathbf{k g})\end{array}$ & $\mathrm{Mp}_{\mathbf{k g}}$ & (kpg & $\underset{\left(\mathbf{R}_{\mathrm{g}}\right)}{\mathrm{M}_{\mathrm{H}}}$ & $\underset{(\mathrm{NEP})}{\mathrm{M}_{\mathrm{NEP}}}$ & $\mathrm{M}_{\mathrm{(g}}$ & 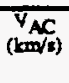 \\
\hline 4.50 & 7.5 & 2.3 & 62 & 7579 & 13 & 12 & 1.16 & 6.6 & 5 & 40 & 2 & 8363 & 3232 & 2855 & 1205 & 4059 & 1072 & 36.3 \\
\hline 4.75 & 8.2 & 2.3 & 60 & 8122 & 14 & 12 & 1.28 & 7.4 & 5 & 40 & 2 & 8340 & 3030 & 2857 & 1168 & 4025 & 1285 & 36.0 \\
\hline 5.00 & 8.9 & 2.3 & 58 & 8653 & 14 & 12 & 1.41 & 8.2 & 5 & 40 & 2 & 8339 & 2869 & 2864 & 1138 & 4003 & 1467 & 35.8 \\
\hline 5.25 & 9.6 & 25 & 55 & 9164. & 15 & 14 & 1.26 & 8.9 & 4 & 36 & 2 & 8204 & 2654 & 2847 & 1061 & 3908 & 1642 & 35.1 \\
\hline 5.50 & 10.4 & 2.8 & 53 & 9650 & 16 & 13 & 1.39 & 9.6 & 4 & 36 & 2 & 8055 & 2463 & 2828 & 1022 & 3850 & 1742 & 34.5 \\
\hline 5.75 & 11.1 & 2.9 & 50 & 10081 & 17 & 13 & 1.52 & 10.3 & 4 & 36 & 2 & 7919 & 2308 & 2811 & 988 & 3799 & 1812 & 34.1 \\
\hline 6.00 & 11.7 & 3.1 & 49 & 10424 & 17 & 16 & 1.22 & 10.8 & 3 & 36 & 2 & 7823 & 2198 & 2801 & 964 & 3765 & 1860 & 33.7 \\
\hline 6.25 & 12.3 & 3.1 & 48 & 10655 & 18 & 16 & 1.27 & 11.2 & 3 & 36 & 2 & 7789 & 2138 & 2799 & 952 & 3750 & 1901 & 33.5 \\
\hline 6.50 & 12.6 & 3.1 & 48 & 10778 & 18 & 16 & 1.29 & 11.4 & 3 & 36 & 2 & 7820 & 2124 & 2807 & 949 & 3756 & 1940 & 33.5 \\
\hline 6.75 & 12.9 & 2.9 & 48 & 10812 & 18 & 16 & 1.27 & 11.5 & 3 & 36 & 2 & 7920 & 2154 & 2826 & 957 & 3783 & 1983 & 33.7 \\
\hline - - $\mathrm{Na}$ & 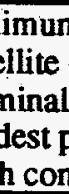 & an & 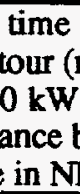 & & & & & & & & & & & & & & & \\
\hline
\end{tabular}

Table 5-4.2 Jupiter Grand Tour Performance Summary (continued)

Requirement: MPL $\geq 1434 \mathrm{~kg}$ (Excess performance are for probes or for two JPO spacecraft )

JGT with (HLV/Centaur + NEP)

\begin{tabular}{|c|c|c|c|c|c|c|c|c|c|c|c|c|c|c|c|c|c|c|}
\hline $\begin{array}{l}F T \\
(y r)\end{array}$ & $\begin{array}{l}\text { FII } \\
(\mathrm{yr})\end{array}$ & $\begin{array}{l}\text { VHL } \\
(\mathrm{km} / \mathrm{s}\end{array}$ & $\begin{array}{c}100 \\
(\mathbf{k w})\end{array}$ & $\underset{(\mathrm{sec})}{\mathrm{SPP}}$ & $\begin{array}{c}P_{R} \\
(\mathbf{k w})\end{array}$ & 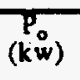 & $\begin{array}{l}T_{L} \\
(y r)\end{array}$ & $\begin{array}{c}\mathrm{T}_{\mathrm{P}} \\
(\mathrm{yr})\end{array}$ & $\mathbf{N}_{0}$ & $\mathbf{N}_{1}$ & $N_{\text {PPU }}$ & $\begin{array}{l}M_{0} \\
\left(\mathbf{k g}_{\mathbf{B}}\right)\end{array}$ & $\mathrm{k}_{\mathrm{p}}$ & (kp & 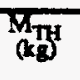 & $\mathrm{M}_{\mathrm{NEP}}$ & $\underset{(\mathrm{rg})}{\mathrm{M}_{\mathrm{pg}}}$ & 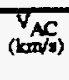 \\
\hline 3.25 & 7.1 & 5.8 & 48 & 8145 & 14 & 12 & 1.28 & 6.8 & 4 & 28 & 2 & 6971 & 2232 & 2587 & 895 & 3482 & 1257 & 30.8 \\
\hline 3.50 & 7.6 & 5.7 & 49 & 8418 & 14 & 12 & 1.31 & 7.1 & 4 & 28 & 2 & 7142 & 2219 & 2615 & 898 & 3513 & 1410 & 30.7 \\
\hline 3.75 & 7.7 & 5.5 & 52 & 8347 & 14 & 13 & 1.21 & 7.0 & 4 & 32 & 2 & 7577 & 2385 & 2681 & 977 & 3658 & 1534 & 30.9 \\
\hline 4.00 & 7.7 & 5.1 & 58 & 8143 & 14 & 12 & 1.34 & 6.8 & 5 & 35 & 2 & 8178 & 2658 & 2774 & 1072 & 3846 & 1674 & 31.4 \\
\hline 4.25 & 7.7 & 4.6 & 66 & 7845 & 13 & 11 & 1.35 & 6.4 & 6 & 36 & 2 & 9079 & 3103 & 2918 & 1190 & 4108 & 1868 & 32.2 \\
\hline 4.50 & 7.4 & 1.6 & 104 & 7521 & 13 & 12 & 1.23 & 6.6 & 9 & 63 & 2 & 13891 & 5517 & 3692 & 1959 & 5651 & 2723 & 37.3 \\
\hline 4.75 & 8.0 & 1.7 & 101 & 8018 & 13 & 13 & 1.21 & 7.3 & 8 & 64 & 2 & 13760 & 5131 & 3673 & 1900 & 5573 & 3056 & 36.7 \\
\hline 5.00 & 8.7 & 1.9 & 97 & 8491 & 14 & 14 & 1.17 & 7.9 & 7 & 63 & 2 & 13600 & 4781 & 3649 & 1828 & 5477 & 3342 & 36.1 \\
\hline 5.25 & 9.4 & 2.0 & 93 & 8918 & 15 & 13 & 1.27 & 8.5 & 7 & 63 & 2 & 13438 & 4486 & 3625 & 1772 & 5397 & 3555 & 35.5 \\
\hline 5.50 & 9.9 & 2.1 & 91 & 9270 & 15 & 15 & 1.17 & 9.0 & 6 & 60 & 2 & 13327 & 4270 & 3609 & 1705 & 5314 & 3743 & 35.1 \\
\hline 5.75 & 10.4 & 2.1 & 89 & 9525 & 16 & 15 & 1.22 & 9.3 & 6 & 60 & 2 & 13314 & 4150 & 3610 & 1682 & 5292 & 3872 & 34.9 \\
\hline 6.00 & 10.8 & 20 & 89 & 9688 & 16 & 15 & 1.24 & 9.6 & 6 & 60 & 2 & 13421 & 4123 & 3631 & 1680 & 5311 & 3987 & 34.9 \\
\hline 6.25 & 11.1 & 1.8 & 91 & 9772 & 16 & 15 & 1.23 & 9.8 & 6 & 60 & 2 & 13692 & 4203 & 3682 & 1701 & 5384 & 4105 & 35.1 \\
\hline 6.50 & 11.3 & 0.9 & 97 & 9809 & 16 & 16 & 1.15 & 10.1 & 6 & 66 & .2 & 14514 & 4602 & 3848 & 1841 & 5689 & 4223 & 36.7 \\
\hline $\begin{array}{r}\text { sh } \\
\text { - } \mathrm{M} \\
\text { - } \mathrm{P}\end{array}$ & 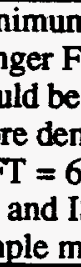 & 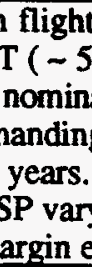 & 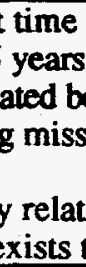 & $t$ & & & & OI & & & & & & & . & $\mathbf{k g}$ & & $\begin{array}{l}\text { FT } \\
\text { ble }\end{array}$ \\
\hline
\end{tabular}


Table 5-5.1 Multiple Mainbelt Asteroid Rendezvous Performance Summary

Requirement: MPL $\geq 1395 \mathrm{~kg}$ (Excess performance are for penetrators)

Rendezvous Sequence

\begin{tabular}{|c|c|c|c|c|c|c|c|}
\hline BODY & Earth & 113-Amulthea & 4-Vesta & 21- Luthetia & 1-Ceres & 68-Leto & 45-Eugenia \\
\hline $\begin{array}{l}\text { RENDEZV- } \\
\text { OUS DATE }\end{array}$ & $\begin{array}{l}7 / 29 \\
2005\end{array}$ & $\begin{array}{l}4 / 22 \\
2007\end{array}$ & $1 / 27$ & $\begin{array}{l}1 / 28 \\
2012\end{array}$ & $\begin{array}{l}8 / 08 \\
2013\end{array}$ & $\begin{array}{l}8 / 18 \\
2015\end{array}$ & $\begin{array}{l}\text { T2/15 } \\
2018\end{array}$ \\
\hline TYPE & & $\mathbf{S X}$ & Vera & $\mathrm{M}$ & G & S & C \\
\hline RADIUS & & 24 & 288 & 34 & 516 & 64 & 122 \\
\hline
\end{tabular}

MMBAR with (Titan IV(SRMU)/Centaur +NEP)

\begin{tabular}{|c|c|c|c|c|c|c|c|c|c|c|c|c|c|c|c|c|c|c|}
\hline$F T$ & FII & $\mathrm{V}_{\mathrm{km} / \mathrm{s} / \mathrm{s}}$ & ${ }_{(\mathrm{kw})}^{10}$ & $\underset{(\mathrm{sec})}{\mathrm{ISP}}$ & $\underset{(\mathbf{k w})}{P_{R}}$ & $\underset{(\mathrm{kw})}{\mathrm{P}_{0}}$ & $\begin{array}{l}T_{L} \\
(y t)\end{array}$ & $\begin{array}{l}T_{\mathrm{P}} \\
(\mathrm{yr})\end{array}$ & $N_{0}$ & $N_{1}$ & $N_{P D U}$ & $\begin{array}{l}\mathrm{M}_{0} \\
(\mathbf{( k g )})\end{array}$ & 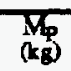 & $\mathrm{M}_{\mathrm{P} P \mathrm{P}}$ & $\underset{(\mathrm{Kg})}{\mathrm{M}_{\mathrm{TH}}}$ & $\overbrace{(\mathrm{NEP})}$ & $\begin{array}{c}\mathrm{M}_{\mathrm{kg}} \\
(\mathrm{kg})\end{array}$ & $\begin{array}{l}V_{A C} \\
\left(\operatorname{lom}^{\prime} / 3\right)\end{array}$ \\
\hline$\sum_{2}$ & 13.4 & 2.9 & 40 & 5335 & 9 & 8 & 1.26 & 5.0 & 5 & 25 & 2 & 7931 & 3185 & 2348 & 890 & 3238 & 1508 & \\
\hline
\end{tabular}

- Multiple rendezvous with highly desirable targets can be adequately accomplished with Titan IV/Centaur.

- Although mass margin is not apparent in the table, it may be obtained with alternative targets and longer

FT

or dropping one target.

- Nominal $P 0=40 \mathrm{~kW}$, ISP $=5300 \mathrm{sec}$. (small compared to other missions)

- Thrust time is modest, but FT is long (on the average 2 years per target) to carry out six rendezvous.

- Best mission for a first NEP application.

Table 5-5.2 Multiple Mainbelt Asteroid Rendezvous Performance Summary (continued) Requirement: MPL $\geq 1395 \mathrm{~kg}$ (Excess performance is for penetrators)

\section{Rendezvous Sequence}

\begin{tabular}{|c|c|c|c|c|c|c|c|}
\hline BODY & Eanh & 4-Vesta & 9-Metis & 21-Luthelis & Io2-1Manam & 1-Ceres & 68-Leto \\
\hline RENDEZN- & $1 / 01$ & 8720 & 901 & 1708 & $3 / 31$ & 8714 & Tims \\
\hline TYPE & & $v$ & $s$ & $M$ & C & G & 5 \\
\hline RABIOS & & 288 & 79 & 34 & 45 & 316 & 64 \\
\hline
\end{tabular}

MMBAR with (HLV/Centaur +NEP)

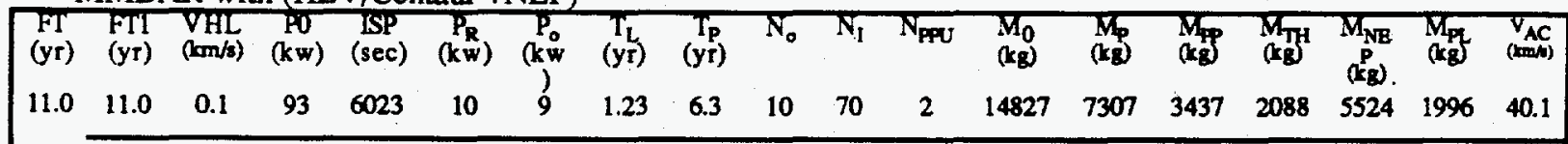

- Performance improvement over (Titan IV(SRMU)/Centaur + NEP) case is not significant.

- Note the larger $V_{A C}$ relative to the (Titan IV/Centaur + NEP) case. The $\Delta V$ cost for doing the mission faster or getting to target with unfavorable phasing is high,

- Nominal $P 0=90 \mathrm{~kW}$, ISP $=6000 \mathrm{sec}$.

- Ample performance margins 
Table 5-6 Comet Nucleus Sample Return Performance Summary

Example Missions - Encounter Dates

\begin{tabular}{|cccc|}
\hline Comet & Launch Date & Comet Date & Earth Return Date \\
\hline Tempel 2 (T2) & $4 / 05 / 2005$ & $9 / 20 / 2008$ & $2 / 24 / 2011$ \\
Tempel 2 (T2) & $6 / 24 / 2004$ & $8 / 26 / 2008$ & $2 / 24 / 2011$ \\
Tsuchinshan 2 (TS2) & $11 / 03 / 2005$ & $5 / 23 / 2008$ & $7 / 20 / 2012$ \\
Tsuchinshan 2 (TS2) & $12 / 16 / 2004$ & $12 / 01 / 2008$ & $7 / 20 / 2012$ \\
Kohoutek (KOH) & $8 / 29 / 2007$ & $8 / 01 / 2010$ & $5 / 31 / 2014$ \\
Kohoutek (KOH) & $10 / 28 / 2006$ & $11 / 13 / 2010$ & $5 / 31 / 2014$ \\
Kopff (KOPF) & $2 / 24 / 2009$ & $7 / 19 / 2012$ & $12 / 20 / 2015$ \\
Kopff (KOPF) & $5 / 19 / 2008$ & $8 / 08 / 2012$ & $12 / 20 / 2015$ \\
Wild 2 (W2) & $10 / 22 / 2009$ & $3 / 05 / 2013$ & $8 / 20 / 2016$ \\
Wild 2 (W2) & $2 / 01 / 2009$ & $3 / 22 / 2013$ & $9 / 19 / 2016$ \\
\hline
\end{tabular}

Requirement: $\quad \mathrm{MPL}_{\mathrm{PL}} \approx 1800 \mathrm{~kg}$ (note: $500 \mathrm{~kg}$ lander $/$ sampler left on comet)

Performance of CNSR with (HLV/Centaur +NEP)

\begin{tabular}{|c|c|c|c|c|c|c|c|c|c|c|c|c|c|c|c|c|c|c|}
\hline $\begin{array}{l}\text { Comet } \\
\text { T2 }\end{array}$ & $\begin{array}{l}\text { FT } \\
\text { (yr) } \\
5.9\end{array}$ & $\begin{array}{c}\mathrm{VF} / \mathrm{k}) \\
(\mathrm{km} / \mathrm{s})\end{array}$ & $\begin{array}{c}\mathbf{P 0} \\
(\mathrm{kw}) \\
86\end{array}$ & $\begin{array}{l}\text { ISP } \\
(\mathrm{sec}) \\
4749\end{array}$ & 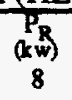 & 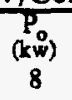 & $\begin{array}{l}T_{L} \\
(y r) \\
1.16\end{array}$ & $\begin{array}{c}T_{p} \\
(y r) \\
3.4\end{array}$ & $\begin{array}{l}N_{0} \\
11\end{array}$ & $\begin{array}{l}N_{I} \\
44\end{array}$ & $\begin{array}{c}N_{\text {PPU }} \\
2\end{array}$ & $\begin{array}{c}M_{0} \\
k 8 g) \\
12651\end{array}$ & $\begin{array}{c}\mathrm{M}_{\mathrm{P}} \\
\mathbf{( 2 8 8} \\
5824\end{array}$ & $\begin{array}{l}\text { M } \\
\text { (198) } \\
3353\end{array}$ & $\begin{array}{l}\mathrm{M}_{\mathrm{TH}} \\
(\mathrm{kg}) \\
1671\end{array}$ & $\begin{array}{l}M_{\text {MEP }} \\
(\mathrm{Rg}) \\
5024\end{array}$ & $\begin{array}{c}M_{p} \\
(k g) \\
1303\end{array}$ & 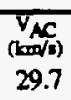 \\
\hline $\mathrm{T} 2$ & 6.7 & 0.8 & 92 & 4773 & 8 & 8 & 1.18 & 3.8 & 12 & 48 & 2 & 14589 & 6866 & 3476 & 1851 & 5328 & 1895 & 30.6 \\
\hline TS2 & 6.8 & 3.1 & 83 & 4525 & 8 & 8 & 1.14 & 2.7 & 11 & 33 & 2 & 11838 & 4994 & 3291 & 1475 & 4766 & 1578 & 25.2 \\
\hline TS2 & 7.6 & 0.9 & 96 & 5067 & 8 & 8 & 1.20 & 3.9 & 12 & 60 & 2 & 14559 & 6659 & 3562 & 1971 & 5533 & 1867 & 31.3 \\
\hline $\mathrm{KOH}$ & 6.7 & 3.5 & 76 & 4560 & 8 & 7 & 1.25 & 2.7 & 11 & 33 & 2 & 10996 & 4513 & 3156 & 1376 & 4531 & 1452 & 24.6 \\
\hline $\mathrm{KOH}$ & 7.6 & 0.8 & 95 & 5076 & 8 & 8 & 1.22 & 3.9 & 12 & 60 & 2 & 14570 & 6548 & 3539 & 1951 & 5491 & 2031 & 30.6 \\
\hline KOPF & 6.8 & 2.4 & 91 & 4493 & 7 & 7 & 1.22 & 2.9 & 13 & 39 & 2 & 12884 & 5800 & 3450 & 1665 & 5115 & 1469 & 27:2 \\
\hline KOPF & 7.6 & 0.9 & 94 & 4960 & 8 & 8 & 1.20 & 3.9 & 12 & 60 & 2 & 14562 & 6674 & 3521 & 1955 & 5476 & 1912 & 30.9 \\
\hline W2 & 6.8 & 2.0 & 95 & 4493 & 7 & 7 & 1.17 & 2.8 & 13 & 52 & 2 & 13464 & 6057 & 3540 & 1838 & 5378 & 1529 & 27.1 \\
\hline W2 & 7.6 & 0.8 & 94 & 4983 & 8 & 8 & 1.20 & 3.8 & 12 & 48 & 2 & 14567 & 6548 & 3525 & 1842 & 5367 & 2152 & 30.0 \\
\hline $\begin{array}{l}\text { Comn } \\
\text { - Dire } \\
\text { 2nd } \\
\text { - Indi } \\
\text { - Nor } \\
\text { - Lar }\end{array}$ & & 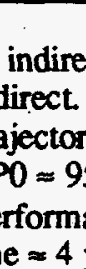 & 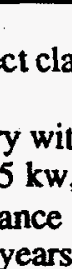 & & & & & & & & & & & & it. & & d. & \\
\hline
\end{tabular}


Table 5-7 1-AU Solar Polar Orbiter Performance Summary

Requirement: $\quad M_{P L} \geq 2500 \mathrm{~kg}$

1-AU Solar Polar Orbiter with (HLV/Centaur +NEP)

\begin{tabular}{|c|c|c|c|c|c|c|c|c|c|c|c|c|c|c|c|c|c|}
\hline $\begin{array}{c}F T \\
(y r)\end{array}$ & $\begin{array}{l}\text { VHL } \\
(\mathrm{km} / \mathrm{s}\end{array}$ & $\begin{array}{c}\mathrm{PO} \\
(\mathrm{kw})\end{array}$ & $\begin{array}{l}\underset{(S P P}{(s e c)}) \\
\text { (a) }\end{array}$ & 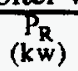 & ${ }_{(\mathrm{rw})}^{P_{0}}$ & $\begin{array}{c}T_{L} \\
(y r)\end{array}$ & $\mathrm{T}_{\mathrm{yr}}$ & $\mathrm{N}_{0}$ & $N_{1}$ & $N_{\text {FU }}$ & $\begin{array}{l}\mathrm{M}_{0} \\
(\mathrm{~kg})\end{array}$ & $\begin{array}{l}\mathrm{Mp}_{\mathrm{p}} \\
(\mathbf{k g})\end{array}$ & 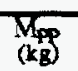 & $\mathrm{M}_{\left(\mathrm{kg}_{\mathrm{H}}{ }^{\prime}\right.}$ & $M_{(\mathbf{N g P})}$ & $\mathrm{M}_{(\mathrm{kg})}$ & $\begin{array}{l}V_{(\mathrm{A})} \\
\left(\mathrm{m}^{\prime}\right)\end{array}$ \\
\hline 5.00 & 2.1 & 92 & 5530 & 9 & 9 & 1.2 & 5.0 & 10 & 60 & 2 & 13292 & 6768 & 3305 & 1943 & 5248 & 1276 & 38.6 \\
\hline 5.25 & 2.0 & 92 & 5704 & 10 & 9 & 1.2 & 5.3 & 10 & 60 & 2 & 13406 & 6718 & 3335 & 1943 & 5278 & 1410 & 38.9 \\
\hline 5.50 & 2.0 & 93 & 5874 & 10 & 9 & 1.2 & 5.5 & 10 & 60 & 2 & 13504 & 6670 & 3365 & 1942 & 5307 & 1527 & 39.2 \\
\hline 5.75 & 1.9 & 93 & 6043 & 10 & 9 & 1.2 & 5.8 & 10 & 60 & 2 & 13589 & 6622 & 3395 & 1942 & 5336 & 1631 & 39.6 \\
\hline 6.00 & 1.8 & 94 & 6212 & 10 & 9 & 1.3 & 6.0 & 10 & 60 & 2 & 13661 & 6572 & $: 25$ & 1941 & 5366 & 1723 & 40.0 \\
\hline 6.25 & 1.8 & 94 & 6355 & 11 & 10 & 1.2 & 6.2 & 9 & 63 & 2 & 13741 & 6527 & 3455 & 1969 & 5424 & 1790 & 40.2 \\
\hline 6.50 & 1.7 & 95 & 6483 & 11 & 11 & 1.2 & 6.3 & 9 & 63 & 2 & 13824 & 6485 & 3485 & 1972 & 5457 & 1882 & 40.3 \\
\hline 6.75 & 1.6 & 96 & 6625 & 11 & 11 & 1.2 & 6.5 & 9 & 63 & 2 & 13887 & 6429 & 3514 & 1973 & 5487 & 1971 & 40.4 \\
\hline 7.00 & 1.6 & 96 & 6795 & 11 & 11 & 1.2 & 6.7 & 9 & 63 & 2 & 13907 & 6345 & 3543 & 1970 & 5513 & 2049 & 40.6 \\
\hline 7.25 & 1.6 & 97 & 7014 & 12 & 11 & 1.2 & 7.0 & 9 & 72 & 2 & 13883 & 6229 & 3574 & 2039 & 5613 & 2041 & 41.0 \\
\hline 7.50 & 1.7 & 97 & 7297 & 12 & 12 & 1.1 & 7.4 & 8 & 72 & 2 & 13817 & 6078 & 3610 & 2026 & 5636 & 2103 & 41.5 \\
\hline 7.75 & 1.8 & 96 & 7234 & 12 & 12 & 1.1 & 6.9 & 8 & 64 & 2 & 13747 & 5698 & 3549 & 1916 & 5465 & 2584 & 38.0 \\
\hline 8.00 & 1.7 & 96 & 7378 & 12 & 12 & 1.2 & 7.1 & 8 & 64 & 2 & 13804 & 5648 & 3569 & 1912 & 5482 & 2674 & 38.1 \\
\hline 8.25 & 1.7 & 96 & 7556 & 13 & 12 & 1.2 & 7.4 & 8 & 64 & 2 & 13844 & 5600 & 3592 & 1907 & 5499 & 2745 & 38.4 \\
\hline 8.50 & 1.6 & 96 & 7750 & 13 & 12 & 1.2 & 7.7 & 8. & 64 & 2 & 13904 & 5578 & 3621 & 1904 & 5525 & 2801 & 39.0 \\
\hline 8.75 & 1.6 & 96 & 7936 & 13 & 12 & 1.3 & 8.1 & 8 & 72 & 2 & 13974 & 5567 & 3652 & 1972 & 5624 & 2783 & 39.6 \\
\hline 9.00 & 1.5 & 96 & 8094 & 13 & 12 & 1.3 & 8.3 & 8 & 72 & 2 & 13999 & 5523 & 3673 & 1968 & 5641 & 2835 & 39.8 \\
\hline 9.25 & 1.5 & 96 & 8252 & 14 & 14 & 1.1 & 8.6 & 7 & 70 & 2 & 14015 & 5478 & 3695 & 1946 & 5641 & 2896 & 40.1 \\
\hline 9.50 & 1.5 & 96 & 8409 & 14 & 14 & 1.2 & 8.9 & 7 & 70 & 2 & 14030 & 5435 & 3717 & 1942 & 5659 & 2936 & 40.4 \\
\hline 9.75 & 1.5 & 96 & 8563 & 14 & 14 & 1.2 & 9.1 & 7 & 70 & 2 & 14051 & 5402 & 3742 & 1940 & 5681 & 2968 & 40.8 \\
\hline 10.0 & 1.4 & 96 & 8710 & 15 & 14 & 1.2 & 9.4 & 7 & 70 & 2 & 14098 & 5388 & 3771 & 1941 & 5711 & 2999 & 41.1 \\
\hline
\end{tabular}


Table 5-8. Interstellar Probe Performance Summary Requirement: $\quad M_{P L} \geq 1000 \mathrm{~kg}$

PIP with (HLV/Centaur + NEP)

\begin{tabular}{|c|c|c|c|c|c|c|c|c|c|c|c|c|c|c|c|c|c|c|}
\hline $\begin{array}{c}F T \\
(\mathrm{yr}) \\
22.0\end{array}$ & $\begin{array}{l}\text { FI1 } \\
\text { (yr) } \\
20.6\end{array}$ & $\begin{array}{l}F 12 \\
(y r) \\
1.4\end{array}$ & $\begin{array}{l}P 0 \\
(\mathbf{k w w}) \\
1000\end{array}$ & $\begin{array}{c}\text { ISP } \\
(\text { sec) } \\
8879\end{array}$ & $\begin{array}{c}P_{R} \\
(\mathbf{k w}) \\
15\end{array}$ & $\begin{array}{c}P_{0} \\
(\mathbf{k w}) \\
15\end{array}$ & $\begin{array}{l}T_{L} \\
(y r) \\
1.1\end{array}$ & $\begin{array}{l}\mathrm{Tp}_{\mathrm{p}} \\
\text { (yr) } \\
11.8\end{array}$ & $\begin{array}{l}N_{0} \\
68\end{array}$ & $\begin{array}{l}N_{1} \\
884\end{array}$ & $\begin{array}{c}\mathrm{N}_{\mathrm{PPU}} \\
2\end{array}$ & $\begin{array}{c}\mathrm{M}_{0} \\
(\mathrm{~kg}) \\
114151\end{array}$ & $\begin{array}{c}\underset{\left(M_{p}\right)}{M_{p}} \\
68878\end{array}$ & $\begin{array}{c}\mathrm{M}_{\mathrm{PP}} \\
(\mathrm{kg}) \\
21999\end{array}$ & $\begin{array}{c}\mathrm{M}_{\mathrm{TH}} \\
(\mathrm{kg}) \\
22202\end{array}$ & $\begin{array}{c}\mathrm{M}_{\mathrm{NEP}} \\
(\mathrm{kg}) \\
\mathbf{4 4 2 0 1}\end{array}$ & $\begin{array}{l}\mathrm{M}_{\mathrm{P}} \\
(\mathrm{kg}) \\
1072\end{array}$ & $\begin{array}{l}V_{A C} \\
(8 m / n) \\
80.5\end{array}$ \\
\hline 22.5 & 21.1 & 1.4 & 1000 & 9131 & 15 & 15 & 1.1 & 12.1 & 66 & 924 & 2 & 113754 & 66853 & 21999 & $22360^{\circ}$ & 44358 & 2543 & 79.3 \\
\hline 23.0 & 21.5 & 1.5 & 1000 & 9375 & 16 & 15 & 1.2 & 12.5 & 65 & 910 & 2. & 113592 & 65091 & 21999 & 22078 & 44076 & 4425 & 78.2 \\
\hline 23.5 & 22.0 & 1.5 & 1000 & 9614 & 16 & 16 & 1.2 & 12.8 & 63 & 882 & 2 & 113627 & 63547 & 21999 & 21696 & 43694 & 6386 & 77.2 \\
\hline 24.0 & 22.4 & 1.6 & 1000 & 9851 & 16 & 16 & 1.1 & 13.1 & 61 & 915 & 2 & 113831 & 62185 & 21999 & 21854 & 43853 & 7793 & 76.3 \\
\hline 24.5 & 22.9 & 1.6 & 1000 & 10087 & 17 & 17 & 1.1 & 13.5 & 60 & 900 & 2 & 114181 & 60979 & 21999 & 21614 & 43613 & 9589 & 75.6 \\
\hline 25.0 & 23.3 & 1.7 & 1000 & 10325 & 17 & 17 & 1.2 & 13.9 & 59 & 944 & 2 & 114658 & 59903 & 21999 & 21893 & 43892 & 10863 & 74.8 \\
\hline 25.5 & 23.8 & 1.7 & 1000 & 10564 & 18 & 18 & 1.1 & 14.3 & 57 & 912 & 2 & 115241 & 58932 & 21999 & 21529 & 43528 & 12781 & 74.2 \\
\hline 26.0 & 24.2 & 1.8 & 1000 & 10802 & 18 & 18 & 1.1 & 14.7 & 56 & 952 & 2 & 115909 & 58042 & 21999 & 21791 & 43790 & 14077 & 73.6 \\
\hline 26.5 & 24.7 & 1.8 & 1000 & 11037 & 18 & 18 & 1.2 & 15.2 & 55 & 935 & 3 & 116635 & 57211 & 21999 & 25689 & 47688 & 11736 & 73.0 \\
\hline 27.0 & 25.1 & 1.9 & 1000 & 11264 & 19 & 19 & 1.2 & 15.6 & 54 & 918 & 3 & 117401 & 56422 & 21999 & 25471 & 47469 & 13510 & 72.4 \\
\hline 27.5 & 25.6 & 1.9 & 1000 & 11481 & 19 & 19 & 1.2 & 16.0 & 53 & 954 & 3 & 118190 & 55665 & 21999 & 25710 & 47709 & 14816 & 71.7 \\
\hline 28.0 & 26.0 & 2.0 & 1000 & 11688 & 19 & 19 & 1.2 & 16.3 & 52 & 936 & 3 & 118995 & 54937 & 21999 & 25489 & 47487 & 16571 & 71.0 \\
\hline 28.5 & 26.5 & 2.0 & 1000 & 11884 & 20 & 20 & 1.2 & 16.7 & 51 & 969 & 3 & 119814 & 54238 & 21999 & 25708 & 47707 & 17869 & 70.2 \\
\hline 29.0 & 26.9 & 2.1 & 1000 & 12070 & 20 & 20 & 1.1 & 17.0 & 50 & 950 & 3 & 120646 & 53570 & 21999 & 25483 & 47482 & 19594 & 69.5 \\
\hline 29.5 & 27.4 & 2.1 & 1000 & 12246 & 20 & 20 & 1.1 & 17.3 & 49 & 931 & 3 & 121493 & 52937 & 21999 & 25262 & 47261 & 21295 & 68.7 \\
\hline 30.0 & 27.8 & 2.2 & 1000 & 12414 & 21 & 20 & 1.2 & 17.6 & 49 & 931 & 3 & 122356 & 52339 & 21999 & 25207 & 47206 & 22811 & 68.0 \\
\hline 22.5 & 21.1 & 1.4 & 750 & 9131 & 15 & 15 & 1.2 & 12.1 & 50 & 700 & 2 & 85316 & 30140 & 18009 & T6843 & 34852 & 324 & 79.3 \\
\hline 23.0 & 21.5 & 1.5 & 750 & 9375 & 16 & 15 & 1.2 & 12.5 & 49 & 686 & 2 & 85194 & 48818 & 18009 & 16601 & 34610 & 1765 & 78.2 \\
\hline 23.5 & 22.0 & 1.5 & 750 & 9614 & 16 & 16 & 1.1 & 12.8 & 47 & 658 & 2 & 85220 & 47660 & 18009 & 16255 & 34264 & 3296 & 77.2 \\
\hline 24.0 & 22.4 & 1.6 & 750 & 9851 & 16 & 16 & 1.1 & 13.1 & 46 & 690 & 2 & 85373 & 46639 & 18009 & 16435 & 34445 & 4290 & 76.3 \\
\hline 24.5 & 22.9 & 1.6 & 750 & 10087 & 17 & 17 & 1.1 & 13.5 & 45 & 675 & 2 & 85636 & 45734 & 18009 & 16224 & 34233 & 5669 & 75.6 \\
\hline 25.0 & 23.3 & 1.7 & 750 & 10325 & 17 & 17 & 1.2 & 13.9 & 44 & 704 & 2 & 85994 & 44927 & 18009 & 16398 & 34408 & 6658 & 74.8 \\
\hline 25.5 & 23.8 & 1.7 & 750 & 10564 & 18 & 17 & 1.2 & 14.3 & 43 & 688 & 2 & 86431 & 44199 & 18009 & 16194 & 34204 & 8028 & 74.2 \\
\hline 26.0 & 24.2 & 1.8 & 750 & 10802 & 18 & 18 & 1.1 & 14.7 & 42 & 714 & 2 & 86932 & 43532 & 18009 & 16356 & 34366 & 9035 & 73.6 \\
\hline 26.5 & 24.7 & 1.8 & 750 & 11037 & 18 & 18 & 1.1 & 15.2 & 41 & 697 & 3 & 87476 & 42908 & 18009 & 19243 & 37252 & 7316 & 73.0 \\
\hline 27.0 & 25.1 & 1.9 & 750 & 11264 & 19 & 19 & 1.1 & 15.6 & 40 & 720 & 3 & 88051 & 42317 & 18009 & 19386 & 37395 & 8339 & 72.4 \\
\hline 27.5 & 25.6 & 1.9 & 750 & 11481 & 19 & 19 & 1.2 & 16.0 & 40 & 720 & 3 & 88643 & 41749 & 18009 & 19334 & 37343 & 9550 & 71.7 \\
\hline 28.0 & 26.0 & 2.0 & 750 & 11688 & 19 & 19 & 1.2 & 16.3 & 39 & 702 & 3 & 89246 & 41203 & 18009 & 19129 & 37139. & 10905 & 71.0 \\
\hline 28.5 & 26.5 & 2.0 & 750 & 11884 & 20 & 20 & 1.1 & 16.7 & 38 & 722 & 3 & 89861 & 40679 & 18009 & 19253 & 37262 & 11920 & 70.2 \\
\hline 29.0 & 26.9 & 2.1 & 750 & 12070 & 20 & 20 & 1.2 & 17.0 & 38 & 722 & 3 & 90485 & 40178 & 18009 & 19207 & 37216 & 13091 & 69.5 \\
\hline 29.5 & 27.4 & 2.1 & 750 & 12246 & 20 & 20 & 1.1 & 17.3 & 37 & 703 & 3 & 91120 & 39703 & 18009 & 19000 & 37010 & 14407 & 68.7 \\
\hline 30.0 & 27.8 & 2.2 & 750 & 12414 & 21 & 20 & 1.2 & 17.6 & 37 & 703 & 3 & 91767 & 39254 & 18009 & 18959 & 36968 & 15544 & 68.0 \\
\hline
\end{tabular}


Table 5-8 Interstellar Probe Performance Summary (continued)

Requirement: $\quad \mathrm{M}_{\mathrm{PL}} \geq 1000 \mathrm{~kg}$

PIP with (HLV/Centaur + NEP)

\begin{tabular}{|c|c|c|c|c|c|c|c|c|c|c|c|c|c|c|c|c|c|c|}
\hline $\begin{array}{l}\text { FT } \\
(y r) \\
22.0\end{array}$ & $\begin{array}{l}\text { FrI } \\
(y r) \\
20.6\end{array}$ & $\begin{array}{l}\text { FI2 } \\
(\mathrm{ys}) \\
1.4\end{array}$ & $\begin{array}{c}80 \\
(\mathbf{k w}) \\
500\end{array}$ & $\begin{array}{c}\text { ISP } \\
(\mathrm{sec}) \\
8879\end{array}$ & $\underset{\left(\begin{array}{c}\mathbf{k} w) \\
15\end{array}\right)}{\mathrm{P}_{\mathrm{R}}}$ & $\underset{(\mathrm{kw})}{\mathrm{Po}}$ & $\begin{array}{l}T_{L} \\
(y r) \\
1.1\end{array}$ & $\begin{array}{l}T_{P} \\
(y r) \\
11.8\end{array}$ & $\begin{array}{l}N_{0} \\
34\end{array}$ & 442 & $\begin{array}{c}N_{\text {PPU }} \\
2\end{array}$ & $\begin{array}{c}\mathrm{M}_{0} \\
(\mathbf{k g}) \\
57076\end{array}$ & $\begin{array}{c}\mathrm{M}_{\mathrm{p}} \\
(\mathrm{kg}) \\
34439\end{array}$ & $\begin{array}{c}M_{\mathrm{PP}} \\
(\mathrm{kg}) \\
13986\end{array}$ & $\begin{array}{c}\mathrm{M}_{\mathrm{TH}} \\
\mathbf{k g})^{2} \\
11127\end{array}$ & $\begin{array}{c}\mathrm{M}_{\mathrm{NEP}} \\
25113\end{array}$ & $\begin{array}{l}\mathrm{MP}_{\mathrm{Pg}} \\
(\mathrm{kg}) \\
-2476\end{array}$ & 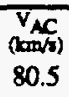 \\
\hline 22.5 & 21.1 & 1.4 & 500 & 9131 & 15 & 15 & 1.1 & 12.1 & 33 & 462 & 2 & 56877 & 33427 & 13986 & 11206 & 25191 & -1741 & 79.3 \\
\hline 23.0 & 21.5 & 1.5 & 500 & 9375 & 16 & 15 & 1.2 & 12.5 & 33 & 462 & 2 & 56796 & 32546 & 13986 & 11125 & 25110 & -860 & 78.2 \\
\hline 23.5 & 220 & 1.5 & 500 & 9614 & 16 & 16 & 1.2. & 12.8 & 32 & 448 & 2 & 56814 & 31774 & 13986 & 10934 & 24919 & 121 & 77.2 \\
\hline 24.0 & 22.4 & 1.6 & 500 & 9851 & 16 & 16 & 1.2 & 13.1 & 31 & 465 & 2 & 56916 & 31093 & 13986 & 11017 & 25003 & 820 & 76.3 \\
\hline 24.5 & 22.9 & 1.6 & 500 & 10087 & 17 & 17 & 1.1 & 13.5 & 30 & 450 & 2 & 57091 & 30490 & 13986 & 10833 & 24819 & 1782 & 75.6 \\
\hline 25.0 & 23.3 & 1.7 & 500 & 10325 & 17 & 17 & 1.2 & 13.9 & 30 & 450 & 2 & 57329 & 29952 & 13986 & 10784 & 24769 & 2608 & 74.8 \\
\hline 25.5 & 23.8 & 1.7 & 500 & 10564 & 18 & 17 & 1.2 & 14.3 & 29 & 464 & 2 & 57621 & 29466 & 13986 & 10859 & 24845 & 3310 & 74.2 \\
\hline 26.0 & 24.2 & 1.8 & 500 & 10802 & 18 & 18 & 1.1 & 14.7 & 28 & 476 & 2 & 57955 & 29021 & 13986 & 10921 & 24907 & 4026 & 73.6 \\
\hline 26.5 & 24.7 & 1.8 & 500 & 11037 & 18 & 18 & 1.2 & 15.2 & 28 & 476 & 3 & 58318 & 28606 & 13986 & 12943 & 26929 & 2783 & 73.0 \\
\hline 27.0 & 25.1 & 1.9 & 500 & 11264 & 19 & 19 & 1.2 & 15.6 & 27 & 459 & 3 & 58701 & 28211 & 13986 & 12761 & 26747 & 3743 & 72.4 \\
\hline 27.5 & 25.6 & 1.9 & 500 & 11481 & 19 & 19 & 1.2 & 16.0 & 27 & 459 & 3 & 59095 & 27833 & 13986 & 12726 & 26712 & 4551 & 71.7 \\
\hline 28.0 & 26.0 & 2.0 & 500 & 11688 & 19 & 19 & 1.2 & 16.3 & 26 & 468 & 3 & 59498 & 27469 & 13986 & 12770 & 26756 & 5273 & 71.0 \\
\hline 28.5 & 26.5 & 2.0 & 500 & 11884 & 20 & 19 & 1.2 & 16.7 & 26 & 468 & 3 & 59907 & 27119 & 13986 & 12738 & 26724 & 6064 & 70.2 \\
\hline 29.0 & 26.9 & 2.1 & 500 & 12070 & 20 & 20 & 1.1 & 17.0 & 25 & 475 & 3 & 60323 & 26785 & 13986 & 12767 & 26753 & 6785 & 69.5 \\
\hline 29.5 & 27.4 & 2.1 & 500 & 12246 & 20 & 20 & 1.2 & 17.3 & 25 & 475 & 3 & 60747 & 26469 & 13986 & 12738 & 26724 & 7554 & 68.7 \\
\hline 30.0 & 27.8 & 2.2 & 500 & 12414 & 21 & 20 & 1.2 & 17.6 & 25 & 475 & 3 & 61178 & 26170 & 13986 & 12711 & 26697 & 8312 & 68.0 \\
\hline 22.0 & 20.6 & 1.4 & 400 & 8879 & 15 & 14 & 1.2 & Tा.8 & 28 & 364 & 2 & 45660 & 27551 & $12030^{\circ}$ & 9001 & 21051 & -2942 & 80.5 \\
\hline 22.5 & 21.1 & 1.4 & 400 & 9131 & 15 & 15 & 1.2 & 12.1 & 27 & 351 & 2 & 45502 & 26741 & 12050 & 8815 & 20865 & -2104 & 79.3 \\
\hline 23.0 & 21.5 & 1.5 & 400 & 9375 & 16 & 15 & 1.2 & 12.5 & 26 & 364 & 2 & 45437 & 26036 & 12050 & 8862 & 20912 & -1511 & 78.2 \\
\hline 23.5 & 22.0 & 1.5 & 400 & 9614 & 16 & 16 & 1.1 & 12.8 & 25 & 350 & 2 & 45451 & 25419 & 12050 & 8685 & 20735 & .703 & 77.2 \\
\hline 24.0 & 22.4 & 1.6 & 400 & 9851 & 16 & 16 & 1.2 & 13.1 & 25 & 375 & 2 & 45532 & 24874 & 12050 & 8850 & 20899 & -241 & 76.3 \\
\hline 24.5 & 22.9 & 1.6 & 400 & 10087 & 17 & 17 & 1.1 & 13.5 & 24 & 360 & 2 & 45672 & 24392 & 12050 & 8677 & 20726 & 554 & 75.6 \\
\hline 25.0 & 23.3 & 1.7 & 400 & 10325 & 17 & 17 & 1.2 & 13.9 & 24 & 360 & 2 & 45863 & 23961 & 12050 & 8637 & 20687 & 1215 & 74.8 \\
\hline 25.5 & 23.8 & 1.7 & 400 & 10564 & 18 & 17 & 1.2 & 14.3 & 23 & 368 & 2 & 46096 & 23573 & 12050 & 8670 & 20720 & 1804 & 74.2 \\
\hline 26.0 & 24.2 & 1.8 & 400 & 10802 & 18 & 17 & 1.2 & 14.7 & 23 & 368 & 2 & 46364 & 23217 & 12050 & 8637 & 20687 & 2460 & 73.6 \\
\hline 26.5 & 24.7 & 1.8 & 400 & 11037 & 18 & 18 & 1.2 & 15.2 & 22 & 374 & 3 & 46654 & 22884 & 12050 & 10306 & 22356 & 1413 & 73.0 \\
\hline 27.0 & 25.1 & 1.9 & 400 & 11264 & 19 & 18 & 1.2 & 15.6 & 22 & 374 & 3 & 46960 & 22569 & 12050 & 10277 & 22327 & 2064 & 72.4 \\
\hline 27.5 & 25.6 & 1.9 & 400 & 11481 & 19 & 19 & 1.1 & 16.0 & 21 & 37.8 & 3 & 47276 & 22266 & 12050 & 10284 & 22334 & 2676 & 71.7 \\
\hline 28.0 & 26.0 & 2.0 & 400 & 11688 & 19 & 19 & 1.2 & 16.3 & 21 & 378 & 3. & 47598 & 21975 & 12050 & 10257 & 22307 & 3316 & 71.0 \\
\hline 28.5 & 26.5 & 2.0 & 400 & 11884 & 20 & 19 & 1.2 & 16.7 & 21 & 378 & 3 & 47926 & 21695 & 12050 & 10232 & 22281 & 3949 & 70.2 \\
\hline 29.0 & 26.9 & 2.1 & 400 & 12070 & 20 & 20 & 1.1 & 17.0 & 20 & 380 & 3 & 48258 & 21428 & 12050 & 10224 & 22274 & 4556 & 69.5 \\
\hline 29.5 & 27.4 & 2.1 & 400 & 12246 & 20 & 20 & 1.2 & 17.3 & 20 & 380 & 3 & 48597 & 21175 & 12050 & 10201 & 22251 & 5172 & 68.7 \\
\hline 30.0 & 27.8 & 2.2 & 400 & 12414 & 21 & 20 & 1.2 & 17.6 & 20 & 380 & 3 & 48942 & 20936 & 12050 & 10179 & 22229 & 5778 & 68.0 \\
\hline
\end{tabular}


Table 5-8. Interstellar Probe Performance Summary (continued)

Requirement: $\quad M_{P L} \geq 1000 \mathrm{~kg}$

PIP with (HLV/Centaur + NEP)

\begin{tabular}{|c|c|c|c|c|c|c|c|c|c|c|c|c|c|c|c|c|c|c|}
\hline $\begin{array}{c}F T \\
(y r) \\
22.0\end{array}$ & $\begin{array}{l}\mathrm{FTl} \\
(\mathrm{yr}) \\
20.6\end{array}$ & $\begin{array}{l}\mathrm{Fr} \\
\text { (yr) } \\
1.4\end{array}$ & $\begin{array}{c}\text { po } \\
(\mathbf{k w}) \\
300\end{array}$ & $\begin{array}{c}\text { ISP } \\
\text { (sec) } \\
8879\end{array}$ & $\begin{array}{c}\mathrm{P}_{\mathbf{R}} \\
(\mathrm{kw}) \\
15\end{array}$ & 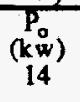 & $\begin{array}{l}T_{L} \\
(y t) \\
1.2\end{array}$ & $\begin{array}{l}\mathrm{T}_{\mathrm{P}} \\
(\mathrm{yr}) \\
11.8\end{array}$ & $\begin{array}{l}\mathrm{N}_{0} \\
21\end{array}$ & $\begin{array}{l}N_{1} \\
273\end{array}$ & $\begin{array}{c}N_{\text {FU }} \\
2\end{array}$ & $\begin{array}{c}\mathrm{M}_{0} \\
(\mathrm{~kg}) \\
34245\end{array}$ & $\begin{array}{c}\mathrm{Mpg}_{\mathrm{pg}} \\
20663\end{array}$ & $\begin{array}{l}M_{p q} \\
(k g) \\
9791\end{array}$ & $\begin{array}{l}\mathrm{M}_{\mathrm{TH}} \\
(\mathrm{kg}) \\
6764\end{array}$ & $\begin{array}{l}M_{\text {NEP }} \\
\left(R_{8}\right)^{2} \\
16555\end{array}$ & $\begin{array}{c}\mathrm{M}_{(\mathrm{g})} \\
-2973\end{array}$ & 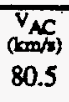 \\
\hline 22.5 & 21.1 & 1.4 & 300 & 9131 & 15 & 15 & 1.2 & 12.1 & 20 & 280 & 2 & 34126 & 20056 & 9791 & 6768 & 16559 & -2489 & 79.3 \\
\hline 23.0 & 21.5 & 1.5 & 300 & 9375 & 16 & 15 & 1.2 & 12.5 & 20 & 280 & 2 & 34078 & 19527 & 9791 & 6719 & 16511 & -1960 & 78.2 \\
\hline 23.5 & 22.0 & 1.5 & 300 & 9614 & 16 & 16 & 1.2 & 12.8 & 19 & 266 & 2 & 34088 & 19064 & 9791 & 6557 & 16348 & -1324 & 77.2 \\
\hline 24.0 & 22.4 & 1.6 & 300 & 9851 & 16 & 16 & 1.2 & 13.1 & 19 & 266 & 2 & 34149 & 18656 & 9791 & 6519 & 16311 & -817 & 76.3 \\
\hline 24.5 & 22.9 & 1.6 & 300 & 10087 & 17 & 17 & 1.1 & 13.5 & 18 & 270 & 2 & 34254 & 18294 & 9791 & 6520 & 16312 & -351 & 75.6 \\
\hline 25.0 & 23.3 & 1.7 & 300 & 10325 & 17 & 17 & 1.2 & 13.9 & 18 & 270 & 2 & 34397 & 17971 & 9791 & 6491 & 16282 & 144 & 74.8 \\
\hline 25.5 & 23.8 & 1.7 & 300 & 10564 & 18 & 17 & 1.2 & 14.3 & 18 & 270 & 2 & 34572 & 17680 & 9791 & 6464 & 16255 & 637 & 74.2 \\
\hline 26.0 & 24.2 & 1.8 & 300 & 10802 & 18 & 18 & 1.2 & 14.7 & 17 & 272 & 2 & 34773 & 17413 & 9791 & 6457 & 16248 & 1112 & 73.6 \\
\hline 26.5 & 24.7 & 1.8 & 300 & 11037 & 18 & 18 & 1.2 & 15.2 & 17 & 272 & 3 & 34991 & 17163 & 9791 & 7670 & 17461 & 366 & 73.0 \\
\hline 27.0 & 25.1 & 1.9 & 300 & 11264 & 19 & 19 & 1.1 & 15.6 & 16 & 288 & 3 & 35220 & 16927 & 9791 & 7785 & 17577 & 717 & 72.4 \\
\hline 27.5 & 25.6 & 1.9 & 300 & 11481 & 19 & 19 & 1.2 & 16.0 & 16 & 288 & 3 & 35457 & 16700 & 9791 & 7765 & 17556 & 1202 & 71.7 \\
\hline 28.0 & 26.0 & 2.0 & 300 & 11688 & 19 & 19 & 1.2 & 16.3 & 16 & 288 & 3 & 35699 & 16481 & 9791 & 7744 & 17536 & 1682 & 71.0 \\
\hline 28.5 & 26.5 & 2.0 & 300 & 11884 & 20 & 19 & 1.2 & 16.7 & 16 & 288 & 3 & 35944 & 16271 & 9791 & 7725 & 17517 & 2156 & 70.2 \\
\hline 29.0 & 26.9 & 2.1 & 300 & 12070 & 20 & 20 & 1.1 & 17.0 & 15 & 285 & 3 & 36194 & 16071 & 9791 & 7681 & 17472 & 2650 & 69.5 \\
\hline 29.5 & 27.4 & 2.1 & 300 & 12246 & 20 & 20 & 1.2 & 17.3 & 15 & 285 & 3 & 36448 & 15881 & 9791 & 7664 & 17455 & 3112 & 68.7 \\
\hline 30.0 & 27.8 & 2.2 & 300 & 12414 & 21 & 20 & 1.2 & 17.6 & 15 & 285 & 3 & 36707 & 15702 & 9791 & 7647 & 17438 & 3567 & 68.0 \\
\hline 22.0 & 20.6 & 1.4 & 200 & 8879 & 15 & 14 & 1.2 & 11.8 & 14 & 182 & 2 & 22830 & 13776 & $7120^{\circ}$ & 4526 & 11646 & -2592 & 80.5 \\
\hline 22.5 & 21.1 & 1.4 & 200 & 9131 & 15 & 14 & 1.2 & 12.1 & 14 & 182 & 2 & 22751 & 13371 & 7120 & 4489 & 11609 & -2229 & 79.3 \\
\hline 23.0 & 21.5 & 1.5 & 200 & 9375 & 16 & 15 & 1.2 & 12.5 & 13 & 182 & 2 & 22718 & 13018 & 7120 & 4457 & 11577 & -1876 & 78.2 \\
\hline 23.5 & 22.0 & 1.5 & 200 & 9614 & 16 & 15 & 1.2 & 12.8 & 13 & 182 & 2 & 22725 & 12709 & 7120 & 4428 & 11548 & -1532 & 77.2 \\
\hline 24.0 & 22.4 & 1.6 & 200 & 9851 & 16 & 15. & 1.2 & 13.1 & 13 & 182 & 2 & 22766 & 12437 & 7120 & 4403 & 11523 & -1194 & 76.3 \\
\hline 24.5 & 22.9 & 1.6 & 200 & 10087 & 17 & 17 & 1.1 & 13.5 & 12 & 180 & 2 & 22836 & 12196 & 7120 & 4364 & 11484 & .844 & 75.6 \\
\hline 25.0 & 23.3 & 1.7 & 200 & 10325 & 17 & 17 & 1.2 & 13.9 & 12 & 180 & 2 & 22932 & 11981 & 7120 & 4344 & 11464 & -513 & 74.8 \\
\hline 25.5 & 23.8 & 1.7 & 200 & 10564 & 18 & 17 & 1.2 & 14.3 & 12 & 180 & 2 & 23048 & 11786 & 7120 & 4326 & 11446 & -185 & 74.2 \\
\hline 26.0 & 24.2 & 1.8 & 200 & 10802 & 18 & 17 & 1.2 & 14.7 & 12 & 180 & 2 & 23182 & 11608 & 7120 & 4310 & 11430 & 143 & 73.6 \\
\hline 26.5 & 24.7 & 1.8 & 200 & 11037 & 18 & 18 & 1.2 & 15.2 & 11 & 187 & 3 & 23327 & 11442 & 7120 & 5179 & 12299 & .414 & 73.0 \\
\hline 27.0 & 25.1 & 1.9 & 200 & 11264 & 19 & 18 & 1.2 & 15.6 & 11 & 187 & 3 & 23480 & 11284 & 7120 & 5164 & 12284 & -89 & 72.4 \\
\hline 27.5 & 25.6 & 1.9 & 200 & 11481 & 19 & 18 & 1.2 & 16.0 & 11 & 187 & 3 & 23638 & 11133 & 7120 & 5151 & 12270 & 235 & 71.7 \\
\hline 28.0 & 26.0 & 2.0 & 200 & 11688 & 19 & 18 & 1.2 & 16.3 & 11 & 187 & 3 & 23799 & 10987 & 7120 & 5137 & 12257 & 555 & 71.0 \\
\hline 28.5 & 26.5 & 2.0 & 200 & 11884 & 20 & 18 & 1.2 & 16.7 & 11 & 187 & 3 & 23963 & 10848 & 7120 & 5124 & 12244 & 871 & 70.2 \\
\hline 29.0 & 26.9 & 2.1 & 200 & 12070 & 20 & 20 & 1.1 & 17.0 & 10 & 190 & 3 & 24129 & 10714 & 7120 & 5138 & 12258 & 1157 & 69.5 \\
\hline 29.5 & $2 i .4$ & 2.1 & 200 & 12246 & 20 & 20 & 1.2 & 17.3 & 10 & 190 & 3 & 24299 & 10587 & 7120 & 5126 & 12246 & 1465 & 68.7 \\
\hline 30.0 & 27.8 & 2.2 & 200 & 12414 & 21 & 20 & 1.2 & 17.6 & 10 & 190 & 3 & 24471 & 10468 & 7120 & 5115 & 12235 & 1768 & 68.0 \\
\hline \multicolumn{19}{|c|}{$\begin{array}{l}\text { - Shuttle-C/Centaur (representative HLV) injection capability to escape is insufficient for this mission } \\
\text { - Mission entails spiral earth escape (starting from a } 1000 \mathrm{~km} \text { circular orbit) } \\
\text { - Delivery capability of Shuttle-C to } 1000 \mathrm{~km} \text { earth orbit is } 50,000 \mathrm{~kg} \text { which restrict the maximum } \\
\text { power to less than } 400 \mathrm{~kW} \text { level } \\
\text { Nominal mission may be } 25 \text { yr flight to } 200 \mathrm{AU} \text {, using } \mathrm{P}_{0}=400 \mathrm{~kW} \& \text { ISP } \approx 10000 \mathrm{sec} \text {. Propulsion time is } 14 \mathrm{yrs} \\
\text { The indication is that the payload delivery can be increased with the increase in power level however the reduction of flight } \\
\text { time approaching } 20 \text { years cannot be realized with the technology level represented in this study (specific mass } \approx 50 \\
\mathrm{~kg} / \mathrm{kW} \text { ) because the intrinsic acceleration level attainable with such a system even with zero payload is inadequate. } \\
\text { Note the non-monotonic MPL increase as a function of flight time. This is the results of integer arithmetic(\# of PPU } \\
\text { increases by one for every } 5 \text { year increase in propulsion time) }\end{array}$} \\
\hline
\end{tabular}




\subsection{NEP SYSTEM REQUIREMENTS}

On the basis of the performance assessment made above, the delineation of NEP system design parameters best suited for various missions are made and summarized in Table 5-9. A range of parameters rather than a single design point is provided. The first entry corresponds to the shortest flight time (except for JGT) and the nominal design point. The second entry represents a fall back design point if the NEP system mass estimates were in error by about a 30\%. One finds the corresponding flight times to be rather unattractive.

Table 5-9. Summary of NEP System Design Parameters

\begin{tabular}{|c|ccccc|}
\hline Mission & UO/P & NEO/P & PLO/ & PLO/P & JGT" \\
\hline Launch Vehicle & HLV & HLV & Titan IV & HLV & Titan IV \\
FT (yr) & $10.5-14$ & $12-15$ & 14.5 & $11.5-14$ & $5-7$ \\
P0 (kW) & $98-92$ & $101-100$ & 56 & $103-99$ & $58-48$ \\
ISP (sec.) & $8400-10000$ & $7800-9500$ & 8400 & $7200-8100$ & $8700-11000$ \\
$N_{0}$ & $7-6$ & $8-7$ & 5 & $9-8$ & $5-3$ \\
NI & $70-72$ & $72-77$ & 40 & $72-64$ & $40-36$ \\
TP (yr) & $8.3-12.3$ & $7.9-10.7$ & 8.0 & $7.0-7.7$ & $8.2-11.5$ \\
Mission Time (yr) & $14-19$ & $14.5-18$ & 16.5 & $13-16$ & $12-15$ \\
\hline
\end{tabular}

\begin{tabular}{|c|cccccc|}
\hline Mission & JGT & MMBAR & MMBAR & CNSR & 1-AU SPO & PIP \\
\hline Launch Vehicle & HLV & Titan IV & HLV & HLV & HLV & HLV \\
& & & & & & (Earth spiral) \\
FT (yr) & $5-6.5$ & 13.5 & 11 & $6.7-7.6$ & $7.6-10$ & 25 \\
P0 (kW) & $97-97$ & 40 & 93 & $92-96$ & 96 & 400 \\
ISP sec.) & $8500-9800$ & 5300 & 6000 & -5000 & $7300-9000$ & 10,000 \\
$N_{0}$ & $7-6$ & 5 & 7 & $12-13$ & $8-7$ & 24 \\
NI & $63-60$ & 25 & 70 & $50-60$ & $64-70$ & 360 \\
T $_{\text {(yr) }}$ & $7.9-10$. & 5 & 6.3 & 4.0 & $7.0-9.5$ & 24 \\
Mission Time (yr) & $11-14$ & 13.5 & 11 & 8 & $20 ?$ & $>25$ \\
\hline
\end{tabular}

\# Missions marginally doable

Given a HLV/Centaur launch vehicle, all missions except the Pioneer Interstellar Probe mission can be performed with a $100 \mathrm{~kW}$ SRPS. The nominal (full-power, life-time) capability of about $(8,15)$ years satisfies the requirements of all planetary missions. Longer lifetime will probablly be required for the 1-AU SPO. The thrusters should be operating at a specific impulse of about 8000 seconds for JGT, the outer planetary missions and the 1-AU SPO. ISP for MMBAR and CNSR are lower, 5000 to 6000 seconds. The thrust subsystem entails fifty to seventy $30-\mathrm{cm}$ thrusters (ion sources) depending on the mission. If the nominal NEP mass characteristics are not met, longer thrust times, longer life times and more thrusters are implied to further compound the design problems.

To do the MMBAR, PLO and JGT missions with a Titan IV/Centaur the optimal power level of the SRPS is about 40-60 kW. A full power time of 8 years is acceptable but the long mission time for PLO dictates a life time of 15 to 16 years. The number of thrusters involved is about 40 maximum.

The design parameters suggested above represent a "near optimal" set of design parameters. The characteristics of low thrust mission performance are such that performance degradation is not severe as one deviates from these design points. If some design parameters are difficuit to accommodate, an imposition of a constraint is a possibility. The degradation in performance due to a constraint, e.g. thrust time, can be made up easily with re-optimized ISP, PO, or FT etc. as long as the constraint is not drastically different from the optimal value. 


\subsection{NEP SYSTEM REQUIREMENTS}

On the basis of the performance assessment made above, the delineation of NEP system design parameters best suited for various missions are made and summarized in Table 5-9. A range of parameters rather than a single design point is provided. The first entry corresponds to the shortest flight time (except for JGT) and the nominal design point. The second entry represents a fall back design point if the NEP system mass estimates were in error by about a $30 \%$. One finds the corresponding flight times to be rather unattractive.

Table 5-9. Summary of NEP System Design Parameters

\begin{tabular}{|c|ccccc|}
\hline Mission & UO/P & NEO/P & PLO/P\# & PLO/P & JGT $^{\#}$ \\
\hline Launch Vehicle & HLV & HLV & Titan IV & HLV & Titan IV \\
FT (yr) & $10.5-14$. & $12-15$ & 14.5 & $11.5-14$ & $5-7$ \\
PO (kW) & $98-92$ & $101-100$ & 56 & $103-99$ & $58-48$ \\
ISP (sec.) & $8400-10000$ & $7800-9500$ & 8400 & $7200-8100$ & $8700-11000$ \\
$N_{0}$ & $7-6$ & $8-7$ & 5 & $9-8$ & $5-3$ \\
$N_{I}$ & $70-72$ & $72-77$ & 40 & $72-64$ & $40-36$ \\
T (yr) & $8.3-12.3$ & $7.9-10.7$ & 8.0 & $7.0-7.7$ & $8.2-11.5$ \\
Mission Time (yr) & $14-19$ & $14.5-18$ & 16.5 & $13-16$ & $12-15$ \\
\hline
\end{tabular}

\begin{tabular}{|c|ccccccc|}
\hline Mission & JGT & MMBAR & MMBAR & CNSR & 1-AU SPO & PIP \\
\hline Launch Vehicle & HLV & Titan IV & HLV & HLV & HLV & HLV \\
& & & & & & (Earth spiral) \\
FT (yr) & $5-6.5$ & 13.5 & 11 & $6.7-7.6$ & $7.6-10$ & 25 \\
P0 (kW) & $97-97$ & 40 & 93 & $92-96$ & 96 & 400 \\
ISP sec.) & $8500-9800$ & 5300 & 6000 & -5000 & $7300-9000$ & 10,000 \\
$N_{0}$ & $7-6$ & 5 & 7 & $12-13$ & $8-7$ & 24 \\
$N_{I}$ & $63-60$ & 25 & 70 & $50-60$ & $64-70$ & 360 \\
TP (yr) & $7.9-10$. & 5 & 6.3 & 4.0 & $7.0-9.5$ & 24 \\
Mission Time (yr) & $11-14$ & 13.5 & 11 & 8 & $20 ?$ & $>25$ \\
\hline
\end{tabular}

\# Missions marginally doable

Given a HLV/Centaur launch vehicle, all missions except the Pioneer Interstellar Probe mission can be performed with a $100 \mathrm{~kW}$ SRPS. The nominal (full-power, life-time) capability of about $(8,15)$ years satisfies the requirements of all planetary missions. Longer lifetime will probablly be required for the 1-AU SPO. The thrusters should be operating at a specific impulse of about 8000 seconds for JGT, the outer planetary missions and the 1-AU SPO. ISP for MMBAR and CNSR are lower, 5000 to 6000 seconds. The thrust subsystem entails fifty to seventy $30-\mathrm{cm}$ thrusters (ion sources) depending on the mission. If the nominal NEP mass characteristics are not met, longer thrust times, longer life times and more thrusters are implied to further compound the design problems.

To do the MMBAR, PLO and JGT missions with a Titan IV/Centaur the optimal power level of the SRPS is about 40-60 kW. A full power time of 8 years is acceptable but the long mission time for PLO dictates a life time of 15 to 16 years. The number of thrusters involved is about $\mathbf{4 0}$ maximum.

The design parameters suggested above represent a "near optimal" set of design parameters. The characteristics of low thrust mission performance are such that performance degradation is not severe as one deviates from these design points. If some design parameters are difficult to accommodate, an imposition of a constraint is a possibility. The degradation in performance due to a constraint, e.g. thrust time, can be made up easily with re-optimized ISP, PO, or FT etc. as long as the constraint is not drastically different from the optimal value. 


\subsection{FLIGHT SYSTEM DESCRIPTION}

\subsection{Flight System Overview}

The NEP planetary mission spacecraft system is conceptually derived from the CRAF/Cassini Mariner Mark II (MMkII) spacecraft design of 15 May 1991. The intent of using the MMkII spacecraft design is to utilize as much heritage as is feasible in order to have greater confidence in the overall mass estimate of the flight system for the purpose of this study.

The MMkII spacecraft is designed for three-axis attitude stabilization using reaction wheels and hydrazine fueled attitude control thrusters to provide control torques about all axes. The spacecraft to Earth communication system utilizes an X-band uplink with an X-band or Ka-band downlink which provides receipt of command transmissions at the spacecraft, the transmission of science and engineering data to the Earth, and radio navigation.

The MMkII spacecraft must be modified for adaptation to a NEP flight system. Primarily, the power, attitude control, and the propulsion subsystems need to be redesigned. While that redesign was not part of this study, mass estimates to accommodate the changes have been included. Clearly, the spacecraft power will be provided by the SP-100 nuclear reactor power subsystem for nominal loads in place of the radioisotope thermoelectric generators (RTGs). A battery can support the flight system loads prior to reactor start-up. The primary chemical propulsion system planned for the large burns of the CRAF and Cassini missions can be removed, because thrust will be provided by the electric propulsion system. However, a small bipropellant propulsion system is maintained for small attitude control maneuvers that may not be able to be provided by the electric propulsion system. Finally, the reaction wheels used for attitude control can be removed.

In order to adapt the MMkIf spacecraft design to a NEP flight system, the bipropellant nitrogen tetroxide/monomethylhydrazine (NTO/MMH) system, designed for control torque maneuvers, requires assessment and redesign as required for pointing and slewing instruments and telecommunications antennas in a timely manner. The high gain antenna will need to be modified with a gimbal so that it can be articulated for minimizing the requirement to point the entire spacecraft to Earth for telecommunications. In addition, the antenna may need to be a deployable Galileo-type high gain antenna for accommodating the launch vehicle adapter and stowage of the flight system in the launch vehicle. The stowed configuration of the spacecraft in Figure 6-2 shows a stowed deployable high gain antenna. Further design studies are required to assess the feasibility of using alternative antennas for specific missions.

The spacecraft is capable of executing on-board stored command sequences for performing trajectory correction maneuvers, pointing of science instruments, collecting and formatting data, and taking self corrective action to on-board problems when there is no immediate earth-based response.

The MMkII spacecraft is attached to an electric propulsion system, powered by the SP-100 nuclear reactor power subsystem, which is extended from the spacecraft by a boom in order to place it at the center of mass of the overall spacecraft. The electric propulsion system is fueled by krypton, and provides the thrust needed to perform the mission. It is also capable of maintaining attitude control of the flight system while in the orbit of the specified target. However, it is assumed that the electric propulsion system cannot provide the required thrust for pointing the instruments or the telecommunications antenna in a timely manner. Thus, the bipropellant propulsion system has been added to provide relatively quick pointing control capability.

Booms are used to extend objects that require location at a distance from the spacecraft main body, such as the magnetometer instrument. Deployable booms are used in order to fit within the limitations of the launch envelope.

A two-axis articulated high precision scan platform is used to mount instruments (for example, the narrow and wide angle cameras) requiring frequent high precision pointing and frequent slewing. A rotating turntable provides a field-of-view (FOV) greater than two pi steradian for fields and particles instruments. Instruments that do not require changes to their look direction are mounted on the spacecraft structure. The 
spacecraft can be modified to carry a probe or penetrator carrying a set of instruments which can be released towards its respective targets.

The mass of the MMkII spacecraft is shown in Table 6-1. The spacecraft lifetime requirement is proportional to the expected mission design. A configuration of the MMkII bus with an outline of the SP100 reactor and electric propulsion system are shown in Figure 6-1. A configuration of the stowed flight system within the Titan IV(SRMU) Centaur payload fairing is shown in Figure 6-2. The conceptual configuration depicted corresponds to the MMBAR mission using a (Titan IV/Centau + NEP).

Table 6-1 Mass Estimate of Spacecraft Subsystems

Table does not include mass estimate for the electric propulsion subsystem, deployable booms, or SP-100 nuclear reactor power subsystem

Subsystem
Structures
Radio Frequency
Power \& Pyro
Command \& Data w/SSR
Attitude Control
Cabling
Chemical Propulsion (dry)
Thermal Control
Mechanical Devices
Antenna
Probe or Penetrator
Probe/Penetrator Support Structure

Mass (kg)

420

45

90

45

120

75

100

40

45

25

200

30

$\begin{array}{ll}\text { Spacecraft Subtotal } & 1235\end{array}$

Contingency (30\%) $\quad 370$

$\begin{array}{ll}\text { Total Mass } & 1605\end{array}$ 


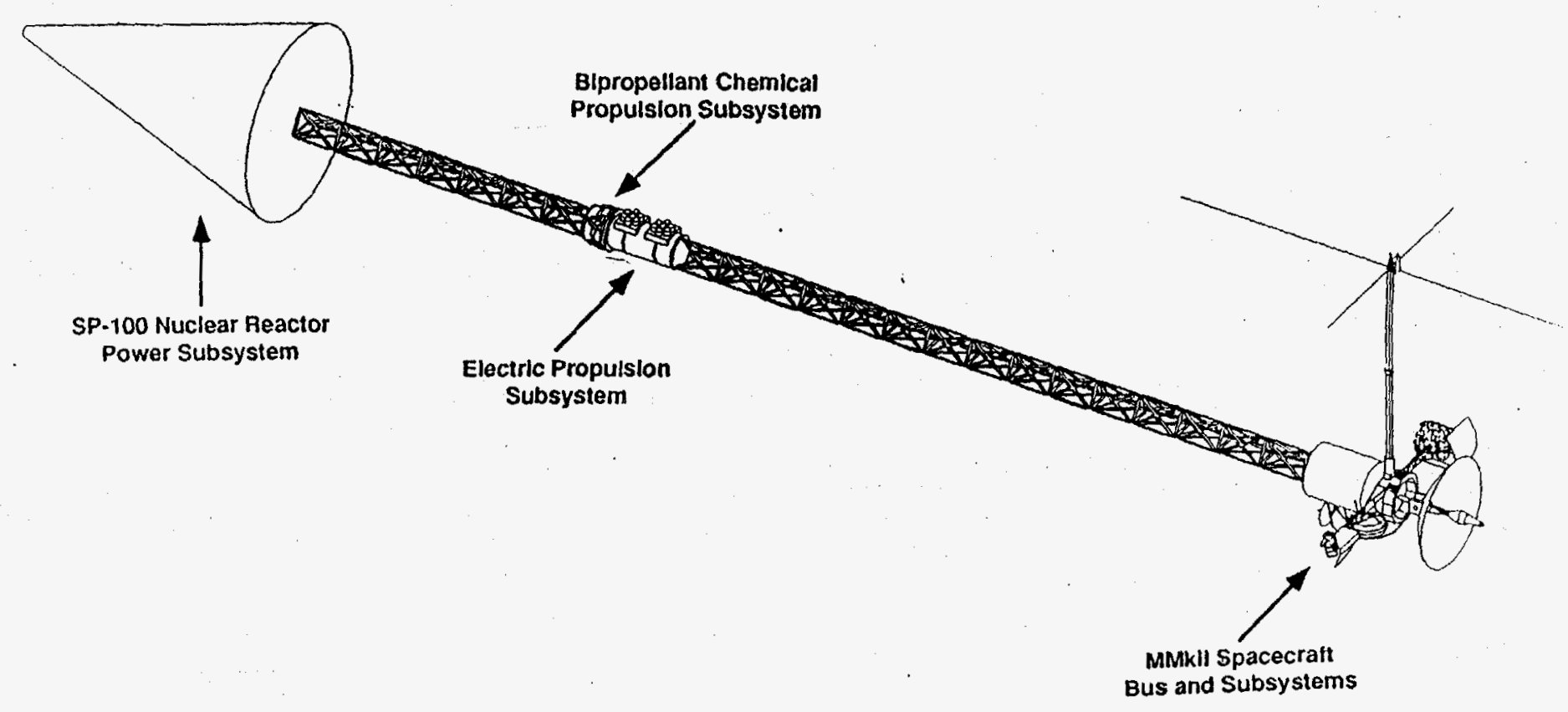

Figure 6-1 Deployed NEP Flight System 

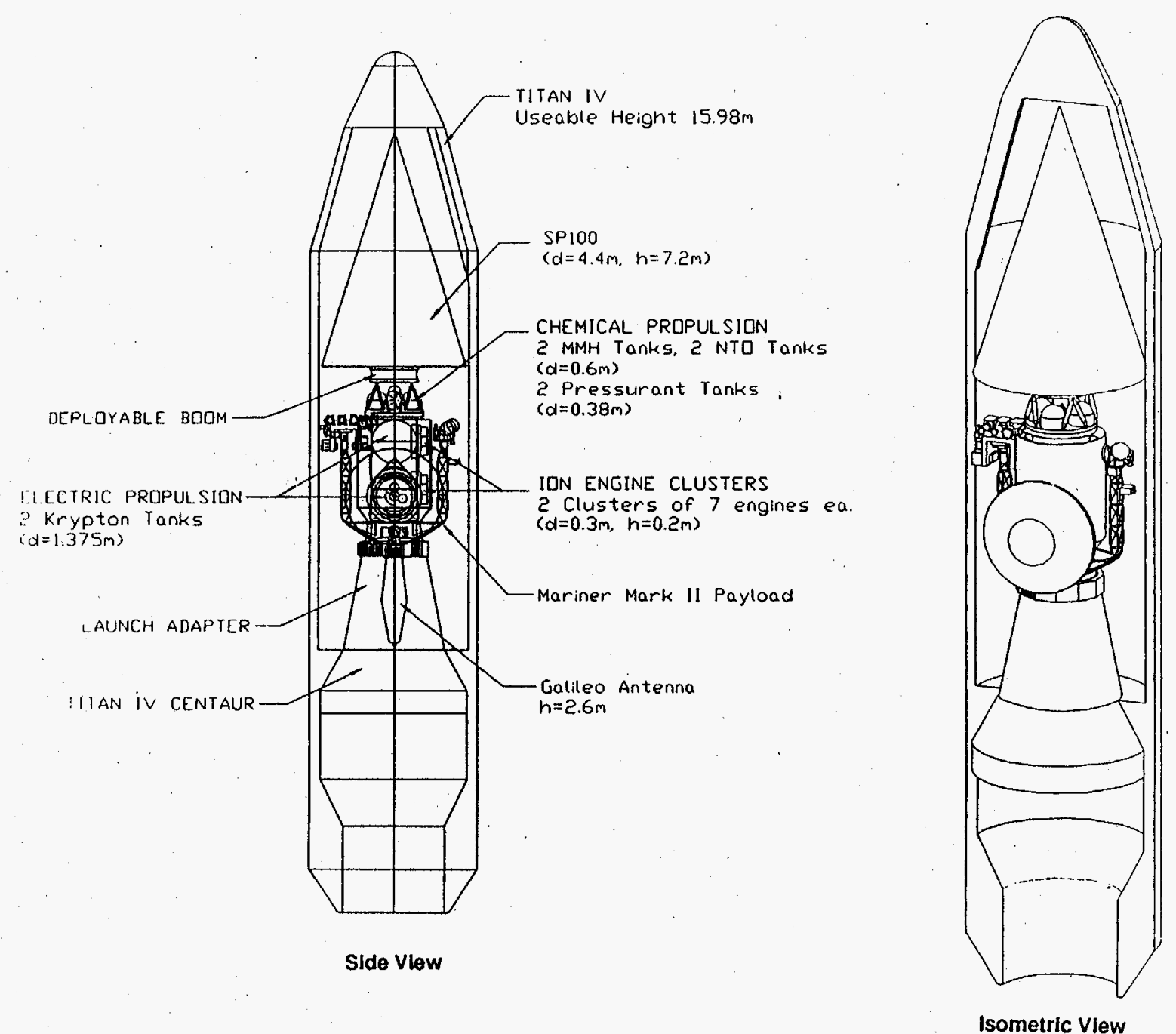

Figure 6-2 Stowed Configuration of NEP Flight System 


\subsection{Subsystem Description.}

\subsubsection{Structure/Configuration}

The structure subsystem mechanically integrates all spacecraft elements into the flight system and provides the launch vehicle (Centaur) adapter. The structure must be designed to support a penetrator or probe, a high precision scan platform for a complement of instruments, and a probe/penetrator relay antenna that is supported from an outrigger on the lower equipment module.

Before the probe/penetrator release, the relay antenna will be unlatched and positioned by the actuator so that its boresight is aligned to view the probe/penetrator. During the course of the encounter, the antenna will point as required to maintain pointing towards the probe/penetrator, up until its limit. The relay attachment to the articulation subsystem is TBD.

The cabling subsystem provides all system wiring interconnections for power, instrumentation, command, grounding, and signal. The system will use GLL-type wire and connectors with shielded wire when required.

\section{Maior Functional Elements}

The structure subsystem consists of the following major elements:

1) Electronics Bus

The bus is part of the primary core structure of the spacecraft and supports the high gain antenna. The bus dual shear plate packaged electronics in a 12 bay configuration and provides a Faraday cage enclosure with appropriate penetrations for cabling.

2) Antenna Structure

The HGA reflector is based on the Galileo deployable HGA. There are two low gain omnidirectional antennas. They are placed strategically on the spacecraft for the purpose of low data rate telecommunications to and from the spacecraft while the flight system is in any attitude orientation.

3) Upper Equipment Module Structure

The upper equipment module will have mounting provisions for a probe or penetrator.

4) Lower Equipment Module Structure

The lower equipment module will have mounting provisions for a relay antenna.

5) High Precision Scan Platform Structure

The high precision scan platform (HPSP) is an aluminum structure which provides for mounting and alignment of remote sensing science instruments, the attitude control target and star tracker, and the fiber optics rotation sensor. Each spacecraft will use the same HPSP although they have different complements of science instrumentation, in addition to the same gimbal system interface, thc same latch truss interface, and the same HPSP outrigger/boom.

6) Tumtable Structure

The turntable is a one-degree-of-freedom structure rotating nominally at one rpm while in orbit about a planet, and a $0.1 \mathrm{rpm}$ during cruise. Short duration's up to $3 \mathrm{rpm}$ while in an orbit will be achievable.

7) Launch Vehicle Adapter

The launch vehicle adapter is an aluminum semi-monoque shell structure which bolts to the Centaur. The scan platforms are latched to the adapter before deployment A pyrotechnic linear separation device provides for separation of the spacecraft from the launch vehicle adapter. Springs provide the impulse to separate the spacecraft from the adapter. The science platform booms are deployed before separation. The magnetometer boom is deployed after separation. 


\subsubsection{Radio Frequency Subsystem}

The MMkII Radio Frequency Subsystem (RFS) is a X/Ka-band and provides for receiving an X-band uplink signal, and transmitting either a non-coherent Ka-band downlink signal whose carrier is phase coherent with the X-band uplink RF carrier.

The spacecraft has a single $4.0 \mathrm{~m}$ diameter Galileo-type deployable antenna in order to meet launch configuration constraints. The HGA is gimbaled in order to point the antenna as needed, because the entire flight system will be too difficult to maneuver for the purpose of telecommunications, and has its boresight along the spacecraft -Z-axis. During most of the mission, the HGA is used for both uplink reception and downlink transmission.

The spacecraft has two low gain antennas (LGAs) with patterns that are symmetric (nominally) approximately with respect to the boresight axis. One LGA has its boresight along the spacecraft -Z-axis and the other LGA has its boresight along the flight system $+Z$-axis. Thus, each LGA provides hemispherical coverage. As the flight system Z-axis is the spacecraft roll axis, the LGA patterns are rollsymmetrical.

The LGAs are used when the HGA cannot be appropriately pointed to Earth. For extended periods during the first two years, a LGA is used for both uplink reception and downlink transmission. A LGA is used throughout the mission up uplink reception in the event of an emergency during which HGA pointing is lost. A LGA is also used for downlink transmission for such an emergency provided the spacecraft is not beyond the range that yields any LGA downlink telemetry. Selection of the LGA to be used at any point in the mission is programmed into the spacecraft CDS.

The RFS includes the following equipment: two redundant Ka-band exciters, redundant 5 W Ka-band traveling wave tube amplifiers (TWTAs), $\mathrm{X}$ to Ka band frequency converter, a Ka-band diplexer, a Ka-band transformer and a Ka-band feed horn antenna. The RFS also includes a Command Detector Unit (CDU) (Mars Observer Design) that receives command data streams from the RF receiver and demodulates and detects the command data stream.

The two LGAs and the HGA are connected to diplexers. A system of three mechanical waveguide RF switches connects the two X-band solid state power amplifiers (XSSPA) to the four diplexer transmit ports. With this system, either XSSPA can be connected to one HGA feed port and/or to either of the two LGAs. With both XSSPAs connected to the HGA, the XSSPAs can be operated simultaneously, increasing the downlink signal strength, if needed, for a few days in each planet orbit. An identical arrangement of three mechanical waveguide RF switches connects the four diplexer receive ports to the two transponder inputs.

\subsubsection{Power/Pyrotechnics}

Power to the overall flight system is provided by the SP-100 nuclear reactor power subsystem. Thus, the primary purpose of the power subsystem is to regulate and convert the power from the SP-100 system; it must also provide the capability to shunt excess power. A shunt regulator unit provides regulation of the bus voltage. It will need to be able to shunt power prcvided by the SP-100 in excess of the spacecraft's needs. The shunt regulator should be functionally redundant internally to insure that no single component failure will cause the regulated DC bus voltage to exceed specifications.

The power must be distributed via the power distribution unit to the user loads at $30 \mathrm{~V}$ DC relative to power return. User loads are connected to the power bus through solid-state power switches which are microprocessor controlled. The power and pyrotechnics subsystem (PPS) will provide for the power to actuate electroexplosive devices via the power switching unit. The PPS will provide the power for minidual drive actuators and for opening and closing science instrument covers. They shall be commanded by solid state power switches.

While the SP-100 reactor provides adequate power, the MMkIl bus is designed with energy storage capability provided by nickel cadmium (NiCd) batteries. The batteries are able to operate for a minimum of 1000 cycles at an average $50 \%$ depth of discharge. A battery control assembly is included to provide the correct voltage for battery charging. 
A power control assembly (PCA) unit is also included. It receives power from the reactor and batteries and delivers power to other elements of the PPS. The PCA contains the PPS microprocessor, including its interfacing circuitry, the battery reconditioning circuitry and many of the PPS telemetry transducers. The PCA routes the DC power bus to the power distribution assembly for power distribution to engineering and science subsystems through solid state power switches. The solid state power switch connects various electrical loads to the spacecraft power bus in response to signals from the PPS microprocessor.

\subsubsection{Command and Data Subsystem}

The command and data subsystem (CDS) provides for the reception, distribution, and execution of uplinked commands and provides the capability for the gathering, formatting, encoding, and downlinking of all spacecraft data (science and engineering).

The CDS is comprised of three main functional blocks and many sub-blocks. The main functional blocks are: a command processor for the reception, distribution, and execution of commands; a telemetry processor for the gathering, formatting, encoding, storing, and downlinking of science and engineering data; a bus interface system to tie the CDS together and to link the CDS with the other engineering subsystems and science instruments.

\section{Command Processor}

The command processor is that part of the CDS which interfaces with the command detector unit of the RFS. The command processor receives and decodes uplink commands. These commands are either acted on internally or passed to some destination outside the CDS. In addition to decoding commands, the command processor also communicates with the other engineering subsystems, and performs fault detection and correction functions for the spacecraft.

\section{Telemetry Processor}

The telemetry processor is that part of the CDS which interfaces with the telemetry modulation unit of the RFS. The telemetry processor is responsible for gathering all the science and engineering data on the spacecraft. It controls the storage and retrieval of data from the tape recorders. It is also responsible for organizing the packaged data into transfer frames, Reed-Solomon encoding these and sending them to the telemetry modulation unit (TMU) for downlink.

\section{Bus Interface System}

The bus interface system provides a communication path between the CDS and the engineering subsystems and science instruments. It also provides the link between the command processor and telemetry processor with the CDS. The communication paths are defined by six serial buses.

\section{Solid State Recorder Subsystem}

A solid state recorder (SSR) is the bulk memory storage device for science data. The solid state recorder is expected to have about $9 \times 10^{8}$ bits of storage remaining at end of mission. Two SSR devices will be flown, with one as a redundant backup unit. The data rates into and out of the SSR will need to be determined. The SSR is capable of simultaneous read and write, and each can be accessed from either half of the CDS.

\subsubsection{Attitude and Articulation Control Subsystem}

The attitude and articulation control subsystem (AACS) is primarily responsible for attitude determination of the spacecraft and pointing control of the scan platforms. The AACS is required to perform the following tasks: 
1) Acquire the sun, following separation from the launch vehicle

2) Ensure proper pointing of the high gain antenna and probe/penetrator relay antenna for proper communications

3) Perform commanded turns of the spacecraft as required for science observations and science instrument calibrations

4) Point the scan platforms towards targets that move relative to inertial space

5) Point the electric propulsion engines as required to provide $\Delta V$ and torques for flight system control

6) Provide optical navigation data to the CDS

7) Provide sufficient engineering data in the telemetry stream to accommodate science data interpretation and ground support operations.

The scan platforms are inertially stabilized using a 3-axis Fiber Optics Rotation Sensor (FORS), which provides the gyro function. Normally, the platform attitude is derived from FORS, with attitude updates provided by the target star tracking function, which is located on the scan platform. The scan platform can be operated in either an inertial or an encoder mode. In the inertial mode, the control is based on the attitude of the platform as measured by FORS, while in the encoder mode, the scan platform is controlled relative to the spacecraft using redundant optical encoders for measuring the azimuth and elevation angles. The encoders are part of the scan platform actuator assembly.

The attitude of the spacecraft is normally derived from the scan platform attitude and the scan platform azimuth and elevation angles as provided by the encoders. Normally, the attitude of the spacecraft is controlled using a reaction control thruster system. The reaction control system, which has not been designed will need to be used for spacecraft control (including rolls and turns) and plafform articulation.

A digital fine sun sensor (DFSS) is also attached on the basebody of the spacecraft. One (redundant) digital 2-axis sensor head gives a FOV centered in the $-Z$ direction. This sensor will normally have the sun within its field of view. The DFSS is used for pointing the HGA, sun acquisition, and for fault protection.

\subsubsection{Propulsion}

The electric propulsion module subsystem provides the impulse required for planetary rendezvous, trajectory maneuvers, and orbit trim maneuvers.

The electric propulsion subsystem consists of four to nine suites of seven $30 \mathrm{~cm}$ krypton engines. The electric propulsion system is placed central to the primary spacecraft and the SP-100 reactor power subsystem as shown in Figure 6-1. Two suites of engines are shown in the figure; other suites of engines would be placed around the structure as needed. Not all engines would be used simultaneously for the sustained trajectory thrust. Another configuration showing more detail of the electric propulsion system is shown in Figure 6-3.

The bipropellant system is used for pointing the spacecraft as required during observation operations. The number of engines and total thrust need to be identified through further studies of the NEP option for planetary missions.

\subsubsection{Thermal Control Subsystem}

To accommodate the large spectrum of thermal environments and overcoming some of the thermal effects of the SP-100 reactor, the spacecraft uses multi-layer insulation (MLI) blankets, sun shades, thermal coatings, louvers, radiators, and heaters to meet subsystem temperature requirements. 
The primary structure of the spacecraft is covered with MLI blankets. Heaters are used as required for bipropellant propulsion subsystem engines. Catalyst beds and thruster lines are heated electrically, and the thruster valves can be heated through the reactor.

Bus temperature control is achieved by using a combination of a louver, and/or radiator, and MLI blankets on each outer shear plate. The design for each bus bay is primarily dependent on the power dissipated in it. For bays with a large variation in power dissipation, a louver is used; for bays with a constant power dissipation, a radiator is used; and bays with a low power dissipation are completely covered with MLI blankets. To strike a balance between thermal and micrometeoroid protection requirements, all the bays except the one used for the radio subsystem utilize louvers with protective covers. The shear plate thickness of radio, and radar bays are sized to provide sufficient micrometeoroid protection. The bus draws replacement heat from electrical heaters.

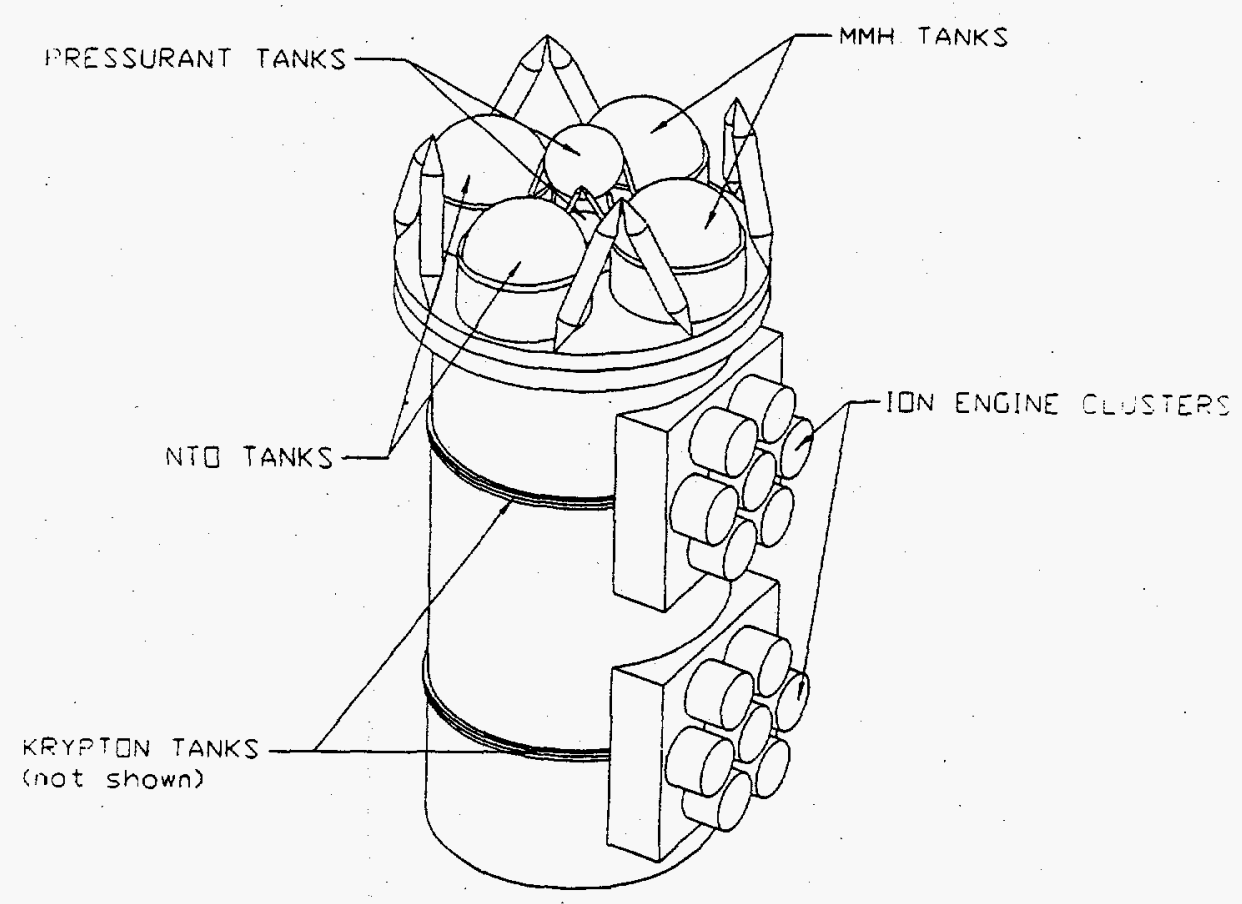

Figure 6-3 Configuration of Electric and Chemical Propulsion System

\subsubsection{Mechanical Devices Subsystem}

The mechanical devices subsystem provides for unlatching and deployment of all appendages, the separation of the spacecraft from the launch vehicle upper stage, and the design and deployment of the magnetometer boom. The adapter separates from the primary bus by means of a super separation device and push-off springs. This subsystem supplies the cover actuators and latch actuators for science instruments.

\subsection{CONCLUSIONS}

The applicability, benefits and requirements of NEP for a set of important space and planetary missions have been examined assuming conservative projections of current SP-100 based space nuclear power technology and 30-cm ring-cusp thruster technology with the expectation that the first NEP mission may be launched in the year $\sim 2005$ (Program New Start year 2000). A preliminary NEP flight system study has 
also been made. A conceptual configuration corresponding to an example MMBAR with (Titan IV/Centaur + NEP) mission has been generated.

\section{Applicability of NEP}

It can be concluded that all of the planetary missions can be performed with reasonable confidence (i.e. a tolerance for a NEP system mass uncertainty of $\sim 30 \%$ ) if a heavy lift launch vehicle with a capability of a Shuttle C/Centaur or better becomes available. The SRPS power level of 100 $\mathrm{kW}$ level can accommodate all missions. The mass characteristic of the nominal dry NEP system is a specific mass of $\sim 57 \mathrm{~kg} / \mathrm{kw}$. The assumed thruster life is 10,000 hours with a margin of $25 \%$ (effectively 7500 hours).

The MMBAR, PLO and JGT missions are feasible with a Titan IV(SRMU)/Centaur launch, but the performance margin is small $(\sim 15 \%)$ for the PLO and JGT. MMBAR using (Titan IV/Centaur +NEP) is the best candidate for early NEP applications. MMBAR mission will return good asteroid science within two years, and can tolerate partial NEP failures because a change of the destinations according to the ability of the system at any stage of the mission is possible.

Additionally, the $100 \mathrm{~kW}$ class NEP is applicable for 1-AU Solar Polar Orbiter mission. Although the thrust time requirement is comparable to the outer planet missions, the lifetime requirement can be longer, $\sim 20$ years. The Pioneer Interstellar Probe mission requires even longer lifetime. The mission may be flown with the SP-100 technology (scaled up to $400 \mathrm{~kW}$ ) but by and large the results are not particularly interesting. One specific requirement of PIP, an ability to make fast trip, can be met only if an advanced low specific mass NEP system is developed.

\section{NEP Benefits}

The NEP mission concepts presented are fundamentally different from the conventional ballistic concepts. Accommodating the common needs of concerned parties to have simplistic performance comparisons between the NEP and ballistic options is not easy or very meaningful. Nonetheless, the comparisons have been made and the superiority of NEP performance for all planetary missions is amply demonstrated. The role of NEP in these concepts are not confined to the transport purposes only. The role of NEP is much broader. Because of the ability of NEP to maneuver, the science content of missions are higher in quality and more comprehensive compared to the ballistic counter parts. Probing of atmosphere, rings, planet and multiple-body (satellites or small bodies) can be made from advantageous orbits.

In addition to far better science, NEP is able to remove most of the perceived difficulties and dilemmas of the missions associated with current MMK If derived ballistic approaches, specifically: 1) NEP enables a Pluto orbiter mission; 2) it provides shorter flight times for Uranus (10.5 years vs 15 years), Neptune (12-15 years vs $>18$ years) and Pluto (11.5-15 years vs $\sim 40$ years for orbiter mission); 3 ) it allows for orbiter missions to the major satellites of Jupiter, Uranus, Neptune, and Pluto vs flybys; and 4) it enables a multiple body mission in the Jupiter Grand Tour and Uranus Orbiter/Probe with one launch, and a multiple asteroid-of-choice mission for asteroid exploration with a single launch; and 5) there are more frequent launch opportunities.

These missions can be performed without an earth spiral escape.

Use of NEP for the space physics missions appear not to be significant. Although it is useful or enable 1-AU Solar Polar Orbiter, the assumed NEP specific mass is inadequate to carry out a fast Pioneer Interstellar Probe mission.

\section{Technology Requirements}

Performance of a sufficient number of important missions are made possible or greatly enhanced by parallel development of the following:

1) $100 \mathrm{~kW}, 10$ year full power, 15 year life mass efficient SP-100 system. 
2) Ion propulsion system consisting of; a) up to ten segmented engines (clusters of five to eight 30-cm ring-cusp thrusters), b) cryogenic Krypton propellant storage/feeding system, c) $100 \mathrm{~kW}$ power processing units, d) thrust control system and e) thrusters with ifetime of 10,000 hours or longer.

3) Mass of 1) \& 2) consistent with the mass characteristics given for the study. SP-100 SRPS system mass of $3700 \mathrm{~kg} @ 100 \mathrm{~kW}$, EP system specific mass of $\sim 20 \mathrm{~kg} / \mathrm{kW}$.

4) HLV system with a capability comparable to or greater than that of Shuttle-C/Centaur

5) NEP-Spacecraft integration

6) Science experiments (new and conventional) possible to conduct in the environment of NEP. 


\section{REFERENCES}

1. Melboume, W. G., and Sauer, C. G., "Performance Comparisons with Pieced Solutions of Planetocentric and Heliocentric Trajectories for Low-Thrust Missions," in Supporting Research and Advanced Development, Space Programs Summary 37-36, Vol. IV, pp. 14-19, Jet Propulsion Laboratory, Pasadena, Calif., December 1965.

2. Stearns, J. W. and Kerrish, D. J., "Solar Powered Electric Propulsion Systems - Engineering and Applications" AIAA Paper no. 66-576, AIAA Second Propulsion Joint Specialist Conference, June 1966.

3. Bender D. F. and Bourke R. D., "Multi-Asteroid Missions Using Solar Electric Propulsion," Paper No. 72-429, AIAA 8th Electric Propulsion conference, April 1972.

4. Hastrup R. C. and Sackett L. L., "Design of Dual Comet Ion Drive Mission," Paper N0. 78-1437, AIAA/AAS Astrodynamics Conference, Palo Alto, Calif., August 1978.

5. Sauer, C. G., "Solar Electric Earth Gravity Assist (SEEGA) missions to The Outer Planets" Paper No. 79-144, AAS/AIAA Astrodynamics conference, Provincetown, Mass., June 1979.

6. Sauer C. G., "SEPS Comet Rendezvous Performance Assessment," Paper No. 80-1685, AAS/AIAA Astrodynamics conference, Danvers, Mass., August 1980.

7. Yen C. L., "Mission Options for the First SEPS Application," Paper No. 81-186, AAS/AIAA Astrodynamics conference, Lake Tahoe, Nevada, August 1981.

8 D. F. Lawden, Optimal Powered Arcs in an Inverse Square Law Field. ARS Jouma1 31, no. 4, S66568 (1961).

9. Melbourne, W. G. and C. G. Sauer, Optimum Thrust Programs for Power-Limited Propulsion Systems, technical report No. 32-118, Jet Propulsion Laboratory, Pasadena, Calif. (1961); Astron. Acta, Vol. VIII, 1962, Fasc, 4.

10. Fimple, W. R., Edelbaum, T. N., et al, "Study of Low-Acceleration Space Transportation System United Aircraft Corporation Report D-910262-3, July 1965.

11. Sauer, C. G., Jr., "Trajectory Analysis and Optimization of a Low-Thrust Solar-Electric Jupiter Flyby Mission, " Paper 67-710, presented at the AIAA Electric Propulsion and Plasmadynamics Conference, Colorado Springs, Colo., September 1967.

12. Sauer, C. G., Jr., "Optimization of A Solar-Electric-Propulsion Planetary Orbiter Spacecraft", AAS paper 68-104, AAS/AIAA Astrodynamics Conference, Jackson, WY, September 3-5, 1968.

13. Hahn, D. W. and F. T. Johnson, "Chebychev Trajectory Optimization Program (CHEBYTOP II)", D180-12916-1, The Boeing Company, Research and Engineèring Division, Seattle, WA, June 1971

14. Kerslaken, W. R., Ignaczak, L. R., "Development and Flight History of SERT II Spacecraft", NASA Technical Memorandum 105636, AIAA-92-3516

15. Sauer, C. G., Jr., "A Comparison of Solar Sail and Ion Drive Trajectories of a Halley Rendezvous Mission", AAS Paper 77-4, AAS/AIAA Astrodynamics Conference, Jackson, WY, September 7-9, 1977.

16. Sackett, L. K., R. C. Hastrup, C. L. Yen and L. J. Wood, "Comet Rendezvous Mission Design Using Solar Electric Propulsion" Paper 79-117, AAS/AIAA Astrodynamic Specialists Conf., Provincetown, MASS, June 25-27, 1979. 
17. Barnett J. W "Nuclear Electric Propulsion Technologies: Overview of the NASA/DoE/DoD Nuclear Electric Propulsion Workshop," Log Nr. 910-116, Eighth Symposium on Space Nuclear Power Systems, Albuquerque, NM, 7-10 January 1990

18. Pluta P. R., Smith M. A. and Matteo D. N. "SP-100, A Flexible Technology for Space Power From 10s to $100 \mathrm{~s}$ of $\mathrm{kWe}$," IECEC-89 Paper No. 899287

19. Beattie J. R. "Ion-Thruster Concept," Hughes Research Laboratories, Presented at the NASA/DoE/DoD Nuclear Electric Propulsion Workshop, Pasadena, CA, 19-22 June 1990.

20. Jones R. M., Editor, "SP-100 Planetary Mission/System Preliminary Design Study - Final Report," Jet Propulsion Laboratory Internal Document, JPL D-2544, June 1986.

21. "OSSA Strategic Plan 1991", Office of Space Science and Applications, NASA, April 6, 1991.

22. "Mission Fact Sheets for NASA/Solar System Exploration Division Program Elements" Documents Prepared for SSES Workshop 1991, February 25-March 1, San Diego, California.

23. "Executive Summary-Report of the Mission Integration and Division Science Panel," Space Physics Strategy Implementation Study, Workshop 2, June 18-21 1990, Bethesda, Maryland.

24. "Final Study Report, Solar Electric Propulsion Vehicle, What Science Could Do With Large Amounts of Power" JPL Document 660-66, August, 1977.

25. F. T. Surber, " High Power Science for NEP Missions", JPL D-10469, January 1993.

26. Mondt J. F., "Development Status of the SP-100 Power System", Paper AIAA-89-2591, 25th Joint Propulsion Conference, July 1989.

27. Jasloff, A. T., Mattleo, D. N. and Bailey, H. S., "SP-100 System Design and Development Progress", IECEC-19, Paper No. 910286.

28. Patterson, M. J. and Verhey, T. R., "5-KW Xenon Ion Thruster Life Test", Paper AIAA-90-2543, AIAA/DGLR/JSASS 21 st International Electric Propulsion Conference, Orlando,Florida,July 1820,1990 .

29. Garner, C. E., et al., "The effects of Nitrogen Addition on Xenon Ion Engine Erosion," AIAA-902591, July 1990.

30. Brophy, J. R. and Garner, C. E., "A 5,000 Hour Xenon Hollow Cathode Life Test," AIAA-91-2122, June 1991.

31. Brophy, J. R., "Near Term, 100 kW Class Ion Engines," AIAA-91-3566, September, 1991.

32. Edlebaum, T. N. "Propulsion Requirements for Controllable Satellites," ARS Journal 1079-1089, Vol. 31, No.8, August 1961.

33. Melbourne, W. G. and Sauer, C. G., Jr., " On Performance Computations with Pieced Solutions of Planetocentric and Heliocentric Trajectories for Low Thrust Missions", Space Program Summary 3736 , Vol IV, Jet Propulsion Laboratory, Pasadena, CA.

34. Yen, C. L., "Main-Belt Asteroid Exploration: Mission Options for the 1990s," Paper AIAA 821463, AAS/AIAA Astrodynamics Conference, San Diego, California, Aug. 9-11, 1982.

35. Sauer, C. G. and Yen, C. L., "Multiple Main-Belt Asteroid Mission Options For A Mariner Mark II Spacecraft," Paper AAS 89-434, AAS/AIAA Astrodynamics Specialist Conference, Stowe, Vermont, Aug. 7-10, 1989. 
36. "ROSETTA The Comet Nucleus Sample Return Mission," Report of the Joint ESA/NASA Science Definition Team, ESA SCI(87)3, Dec. 1987.

37. Feingold, H., "Comet Nucleus Sample Retum," Journal of the British Interplanetary Society, Vol. 37, No. 8, Aug. 1984, pp 388-393.

38. Sauer, C. G., "Current Trajectory Options For Comet Nucleus Sample Return Mission", Paper AAS 91-474, AAS/AIAA Astrodynamics Specialist Conference, Durango, Colorado, Aug. 19-22, 1991

39. Penn, $T$. , "Interstellar probe spacecraft System Design Input for Code ES Strategic Implementation Study", Interoffice Memorandum 3132-90-205, Jet Propulsion Laboratory, Apr. 30, 1990. 